\title{
Relativistic-Covariant Energy-Momentum Tensor for Homogeneous Anisotropic Dispersive Media
}

\author{
Alfred Wünsche \\ Institut für Physik, Humboldt-Universität, Berlin, Germany \\ Email: alfred.wuensche@physik.hu-berlin.de
}

How to cite this paper: Wünsche, A. (2021) Relativistic-Covariant Energy-Momentum Tensor for Homogeneous Anisotropic Dispersive Media. Journal of Modern Physics, 12, 1866-1921.

https://doi.org/10.4236/jmp.2021.1213108

Received: October 7, 2021

Accepted: November 27, 2021

Published: November 30, 2021

Copyright $\odot 2021$ by author(s) and Scientific Research Publishing Inc. This work is licensed under the Creative Commons Attribution International License (CC BY 4.0).

http://creativecommons.org/licenses/by/4.0/

\section{(c) (i) Open Access}

\begin{abstract}
A new relativistically covariant approach is discussed for the derivation of local conservation theorems for homogeneous anisotropic and, in particular, dispersive media. We start from a three-dimensional operator equation for the electric field and obtain mainly by coordinate-invariant methods the results basically expressed by the slowly varying amplitudes of the electric field. Apart from local energy and momentum conservation formulated by the energy-momentum conservation, we find a local conservation theorem for the action which is more general and which is the only one which remains also true for inhomogeneous media.
\end{abstract}

\section{Keywords}

Spatial and Frequency Dispersion, Permittivity Tensor, Group Velocity, Cold Plasma, Coordinate-Invariant Methods, Lorentz Group, Lorentz Invariants

\section{Some Notations}

Three-dimensional vectors: bold letters, e.g., $\boldsymbol{a}, \boldsymbol{b}, \boldsymbol{c}, \cdots$;

$\boldsymbol{a} \boldsymbol{b}$ scalar products, $[\boldsymbol{a}, \boldsymbol{b}]$ vector products, $\boldsymbol{a} \cdot \boldsymbol{b}$ dyadic products;

Latin letters as indices, three-dimensional, e.g., $i=1,2,3, \varepsilon_{i j k}$ is Levi-Civita pseudo-tensor;

Greek letters as indices, four-dimensional, e.g., $\mu=1,2,3,4$;

$\alpha \equiv 4 \pi \quad$ (for $C G S$ or Gauss system of units, $\alpha$ is often in the denominator of formulae).

\section{Introduction}

The four-dimensional energy-momentum tensor was introduced 1913 by Eins- 
tein with notation $\Theta_{\mu v}$ in [1] (republished in [2]) for the relativistically covariant generalization of the local conservation theorems of energy and momentum in differential form ${ }^{1}$. Three-dimensional parts of this tensor (Maxwell stress tensor) were already known before and used earlier. These local conservation theorems of energy and momentum are as it is well known a consequence of the homogeneity or translation invariance of (three-dimensional Euclidean) space and of time.

Beginning from 1915 Einstein published his General relativity theory (see, e.g., [2]) in which the symmetric energy-momentum tensor $T_{\mu \nu}$ is a kind of source term for curvature of space-time expressed by the symmetric Ricci tensor $R_{\mu v}$ which results from Riemann curvature tensor which is a four-valent tensor by contraction over two of its four indices [3] [4]. Due, in particular, to Pauli [5] [6] who wrote in his very young year of 21 an encyclopedic article about the new General relativity theory which requires a symmetric energy-momentum tensor a long discussion of the right energy-momentum tensor, the symmetric Abraham tensor or the non-symmetric Minkowski tensor, began where Pauli brought arguments in favor of the Abraham tensor. However, the Abraham tensor was only derived for isotropic media without taking into account dispersion and a more general symmetric tensor for all subgroups of the three-dimensional orthogonal group cannot exist. Pauli obviously recognized his incorrectness and in a later republication of his article shortly before his death he corrected it in the remark (see Section 13 here). Now the problem of the right energy-momentum tensor for media seems to be decided in favor of the Minkowski tensor.

Our main older sources for considerations to the energy-momentum tensor and its parts were, in particular, Landau and Lifshits [3] [7], Agranovich and Ginzburg [8] [9] [10] and Silin and Rukhadze [11] where, in particular, the dispersion was taken into account and apart from already cited encyclopedic article of Pauli, the work of Tolman [12], Sommerfeld [13], von Laue [14], Fock [15], Skobeltsyn [16] and Ugarov [17] [18], the (astonishingly modern) work of Tamm [19] (1 $1^{\text {st }}$ Ed. 1929) and the monographs of Møller [20] and of Jackson [21] for the older development of electrodynamics and Relativity theory. The number of interesting text-books with treatment of these topics grew rapidly in the following time among them, e.g., [22] [23] [24] [25] [26] which organically take into account dispersion. Long before but less known is the coordinateinvariant approach which was developed, first, mainly in the work of F.I. Fyodorov [27] [28] [29] (see also [30] [31]). Many articles of later time did not use these methods for reflection and refraction problems where, in particular, they are advantageous and derived with other (mostly coordinate) methods new re-

${ }^{1}$ Contravariant and covariant components of tensors are not yet distinguished in this article by upper and lower indices and $\Theta_{\mu v}$ is meant as contravariant tensor. The corresponding covariant energy-momentum tensor with notation $T_{\mu v}$ is introduced there by the relation $T_{\mu v}=g_{\mu \alpha} g_{v \beta} \Theta_{\alpha \beta}$ with $g_{\mu v}$ the covariant metric tensor ( $\gamma_{\mu v}$ corresponding contravariant tensor). Both these tensors are symmetric ones. All this was prepared for the transition to curvilinear coordinates which is discussed in Mathematical Part II written together with M. Grossmann. 
sults or rederived older results. In [32] [33] were given new arguments in favor of the Abraham tensor.

Apart from Abraham or Minkowski tensor there exist yet other serious problems with the energy-momentum tensor. As it is well known, the energy-momentum tensor is not uniquely determined by the requirement to satisfy a differential conservation theorem, e.g., [3] [20]. Practically, one has to do in every case of a calculated energy-momentum tensor with a whole class of equivalent energy-momentum tensors. One main use of this non-uniqueness is the possibility to remove the highly oscillatory terms in the approximation to waves with slowly varying amplitudes (beams) if we first insert the whole electromagnetic field with a positive and corresponding negative peak in the frequency distribution. We discuss this in Section 14 but mention already here that the energy-momentum tensor for media (only vacuum excluded) is basically non-symmetric and that by using the non-uniqueness it cannot be reduced to a symmetric one. Another difficulty for dispersive media is that their energy-momentum tensor cannot be derived starting from a Lagrange function as consequence of translation invariance of the medium in space and time (Noether theorem) as it is standard for electrodynamics of the vacuum. For inhomogeneous media the energy-momentum tensor does not exist at all in a local conservation law.

In present article we consider first the equations of macroscopic electrodynamics as averaged from microscopic electrodynamics (Section 2) then the constitutive equations in the concept of media with spatial and frequency dispersion and the symmetry of the permittivity tensor for neglect of dissipation (Section 3) and its symmetry under presence of discrete symmetries (space inversion, time inversion and their product, Section 4). Then we derive a three-dimensional operator equation for the electric field (Section 5) which is relativistically covariant but on the first glance it may seem to be paradoxical that this is possible. From this operator equation we derive a local conservation theorem for action (Section 6) and for energy and momentum (Section 7) and discuss the obtained energy-momentum tensor (Section 8). A peculiarity of our approach is that we obtain basically all results expressed by the electric field alone and after limiting transition to plane monochromatic waves we make the transition to more usual representation by the electric and magnetic field (Section 9). Then we consider the role which the group velocity plays (Section 10). After this we discuss the neglect of dispersion and the calculation of the group velocity in this case (Section 11). Next, we consider the special case of a cold plasma (Section 12). In the discussion of controversial opinions to local conservation of angular momentum we show that a complete symmetry of the four-dimensional energy-momentum tensor is not necessary but only symmetry of the stress tensor (Section 13). The non-uniqueness of the energy-momentum tensor is considered under new aspects (Section 14). Connected with the general non-symmetry of the energy-momentum tensor arise some difficulties for the General relativity theory (Section 15) and, finally, we mention some possibilities for generalizations of the discussed material (Section 16). In two Appendices we have separated the deri- 
vation of the relativistic covariance of our approach and the transformation of the energy-momentum tensor under special Lorentz transformations. A short paper to some of these problems can be found in [34] (see "Remark" and "Acknowledgements" at the end of present article).

\section{Maxwell Equations of Macroscopic Electrodynamics in Two Concepts}

The basis of our derivations is the following Maxwell equations of macroscopic electrodynamics

$$
\begin{aligned}
& {[\nabla, \boldsymbol{E}(\boldsymbol{r}, t)]+\frac{1}{c} \frac{\partial}{\partial t} \boldsymbol{B}(\boldsymbol{r}, t)=\mathbf{0}, \quad \nabla \boldsymbol{B}(\boldsymbol{r}, t)=0} \\
& {[\nabla, \boldsymbol{B}(\boldsymbol{r}, t)]-\frac{1}{c} \frac{\partial}{\partial t} \boldsymbol{E}(\boldsymbol{r}, t)=\frac{4 \pi}{c} \frac{\partial}{\partial t} \boldsymbol{P}(\boldsymbol{r}, t), \quad \nabla \boldsymbol{E}(\boldsymbol{r}, t)=-4 \pi \nabla \boldsymbol{P}(\boldsymbol{r}, t),}
\end{aligned}
$$

where $\boldsymbol{E}$ and $\boldsymbol{B}$ are the averaged electric field $\boldsymbol{E}^{\text {micro }}$ and magnetic field $\boldsymbol{B}^{\text {micro }}$ of microscopic electrodynamics in the electron theory of Lorentz [35] ${ }^{2}$ (republ. in [36]; see also [37] [38]) in the sense of the transition from microscopic to macroscopic electrodynamics ([7], de Groot and Suttorp [39], (II. section 3))

$$
\boldsymbol{E}(\boldsymbol{r}, t) \equiv \overline{\boldsymbol{E}^{\text {micro }}}(\boldsymbol{r}, t), \quad \boldsymbol{B}(\boldsymbol{r}, t) \equiv \overline{\boldsymbol{B}^{\text {micro }}}(\boldsymbol{r}, t) .
$$

This transition can include different averaging processes, for example, spatial, temporal and statistical ones (denoted by overlining of the corresponding quantity). The averaged microscopic current density $\boldsymbol{j}^{\text {micro }}$ and charge density $\rho^{\text {micro }}$ are expressed by only one macroscopic quantity $\boldsymbol{P}$, called polarization, in the rank of following definition

$$
\overline{\boldsymbol{j}^{\text {micro }}}(\boldsymbol{r}, t) \equiv \frac{\partial}{\partial t} \boldsymbol{P}(\boldsymbol{r}, t), \overline{\rho^{\text {micro }}}(\boldsymbol{r}, t) \equiv-\nabla \boldsymbol{P}(\boldsymbol{r}, t),
$$

where the necessary validity of the continuity equation for microscopic current and charge densities

$$
\nabla \overline{\boldsymbol{j}^{\text {micro }}}(\boldsymbol{r}, t)+\frac{\partial}{\partial t} \overline{\rho^{\text {micro }}}(\boldsymbol{r}, t)=0,
$$

is taken into account. Such an identification is possible in almost all cases with exception of some static cases (e.g., electrostatics, stationary currents, magnetostatics) which have to be considered in this concept as limiting cases. As usual, we define the "electric induction" ${ }^{2} \boldsymbol{D}(\boldsymbol{r}, t)$ by

$$
\boldsymbol{D}(\boldsymbol{r}, t) \equiv \boldsymbol{E}(\boldsymbol{r}, t)+4 \pi \boldsymbol{P}(\boldsymbol{r}, t) .
$$

From vectorial equations in (2.1) follows then by forming the divergence ${ }^{2}$ Lorentz denotes microscopic fields with small letters corresponding to the Capital letters commonly used in macroscopic electrodynamics (but $\boldsymbol{d}$ instead of $\boldsymbol{e}$ for microscopic electric field). Most authors use $\boldsymbol{H}^{\text {micro }}$ instead of $\boldsymbol{B}^{\text {micro }}$ but since there is no difference between them in microscopic theory this is only of some didactic importance.

${ }^{3}$ We follow here in terminology Landau and Lifshits [7] (Russian editions) to distinguish the "electric induction" from such notions as, e.g., "dielectric displacement" which are mostly used in a more special sense. 


$$
\frac{\partial}{\partial t} \nabla \boldsymbol{B}(\boldsymbol{r}, t)=\mathbf{0}, \quad \frac{\partial}{\partial t} \boldsymbol{\nabla} \boldsymbol{D}(\boldsymbol{r}, t)=0,
$$

that are the scalar equations in (2.1) differentiated with respect to the time. Therefore with exclusion of the static limiting case, the scalar equations from (2.1) in

$$
\begin{array}{ll}
{[\nabla, \boldsymbol{E}(\boldsymbol{r}, t)]+\frac{1}{c} \frac{\partial}{\partial t} \boldsymbol{B}(\boldsymbol{r}, t)=0,} & \nabla \boldsymbol{B}(\boldsymbol{r}, t)=0, \\
{[\nabla, \boldsymbol{B}(\boldsymbol{r}, t)]-\frac{1}{c} \frac{\partial}{\partial t} \boldsymbol{D}(\boldsymbol{r}, t)=0,} & \nabla \boldsymbol{D}(\boldsymbol{r}, t)=0,
\end{array}
$$

are redundant and the only field equations to take into account are the vectorial equations.

One can often find in literature forms of the equations of macroscopic electrodynamics where the averaged current and charge density are not fully included only into one quantity $\boldsymbol{P}(\boldsymbol{r}, t)$ called polarization and defined by (2.3) but into some different quantities, for example, electric polarization in a more special sense $\boldsymbol{P}^{\prime}(\boldsymbol{r}, t)$ and magnetization $\boldsymbol{M}(\boldsymbol{r}, t)$ according to, e.g., de Groot and Suttorp [39] and Bloembergen [40] (chap. 3, Eqs. (3.3), (3.5)) (the "Netherland school" together with H.A. Lorentz) but also many other authors

$$
\begin{aligned}
& \overline{\boldsymbol{j}^{\text {micro }}}(\boldsymbol{r}, t) \equiv \frac{\partial}{\partial t} \boldsymbol{P}^{\prime}(\boldsymbol{r}, t)+c[\boldsymbol{\nabla}, \boldsymbol{M}(\boldsymbol{r}, t)]+\cdots, \\
& \overline{\rho^{\text {micro }}}\left((\boldsymbol{r}, t) \equiv-\boldsymbol{\nabla} \boldsymbol{P}^{\prime}(\boldsymbol{r}, t)+\cdots,\right.
\end{aligned}
$$

and in nonlinear optics sometimes additionally into electric quadrupole density and higher electric and magnetic multipole densities (indicated by additional points). They can be joined then in different ways with the electric field $\boldsymbol{E}(\boldsymbol{r}, t)$ and magnetic field $\boldsymbol{B}(\boldsymbol{r}, t)$ to new quantities, for example, to a more special dielectric displacement $\boldsymbol{D}^{\prime}(\boldsymbol{r}, t)$ and to a new field $\boldsymbol{H}(\boldsymbol{r}, t)$ as follows

$$
\boldsymbol{D}^{\prime}(\boldsymbol{r}, t) \equiv \boldsymbol{E}(\boldsymbol{r}, t)+4 \pi \boldsymbol{P}^{\prime}(\boldsymbol{r}, t), \quad \boldsymbol{H}(\boldsymbol{r}, t) \equiv \boldsymbol{B}(\boldsymbol{r}, t)-4 \pi \boldsymbol{M}(\boldsymbol{r}, t),
$$

which obey then the following field equations instead of (2.7)

$$
\begin{aligned}
& {[\nabla, \boldsymbol{E}(\boldsymbol{r}, t)]+\frac{1}{c} \frac{\partial}{\partial t} \boldsymbol{B}(\boldsymbol{r}, t)=\mathbf{0}, \quad \boldsymbol{\nabla} \boldsymbol{B}(\boldsymbol{r}, t)=0,} \\
& {[\boldsymbol{\nabla}, \boldsymbol{H}(\boldsymbol{r}, t)]-\frac{1}{c} \frac{\partial}{\partial t} \boldsymbol{D}^{\prime}(\boldsymbol{r}, t)=\mathbf{0}, \quad \boldsymbol{\nabla} \boldsymbol{D}^{\prime}(\boldsymbol{r}, t)=0 .}
\end{aligned}
$$

The field $\boldsymbol{H}(\boldsymbol{r}, t)$ is mostly called magnetic field but it is not the averaged microscopic magnetic field $\overline{\boldsymbol{B}^{\text {micro }}}(\boldsymbol{r}, t)$ and therefore not the "genuine" magnetic field [7] (chap IV, section 29, after Eq. (29.8)) (in our treatment with equations (2.7) we have $\boldsymbol{H} \equiv \boldsymbol{B}$ and $\boldsymbol{M}$ is included into $\boldsymbol{P}$ ). However, the greater symmetry of (2.10) in comparison to (2.7) is deceptive and to fix the separation (2.8) is then difficult and not fully unique without additional conventions, in particular, for high frequencies under presence of dispersion in the constitutive equations. To see this we recommend to read also the very instructive section 79 in Landau and Lifshits [7]. All such more special schemes can be transformed to the general scheme which we prefer and which is characterized by Equations 
(2.3), (2.5) and (2.7) and by constitutive relations considered in next Section and only some static cases have to be dealt with then as limiting cases.

\section{Linear Constitutive Equations for Homogeneous Anisotropic Dispersive Media}

The Maxwell equations of macroscopic electrodynamics (2.1) or (2.7) form a closed system of equations only together with constitutive equations which depend on the kind of the considered medium. We discuss this now.

The general linear constitutive relation between the electric induction $\boldsymbol{D}(\boldsymbol{r}, t)$ and the electric field $\boldsymbol{E}(\boldsymbol{r}, t)$ for spatially and temporally homogeneous media is (summation convention over equal indices; $\boldsymbol{\rho} \equiv \boldsymbol{r}-\boldsymbol{r}^{\prime}, \tau=t-t^{\prime}$; $\exp \left(-\rho \nabla-\tau \frac{\partial}{\partial t}\right)$ is displacement operator of arguments of a function of $(\boldsymbol{r}, t)$ to $(\boldsymbol{r}-\boldsymbol{\rho}, t-\tau))$

$$
\begin{aligned}
D_{i}(\boldsymbol{r}, t) & =\int \mathrm{d}^{3} \rho \wedge \mathrm{d} \tau \hat{\varepsilon}_{i j}(\boldsymbol{\rho}, \tau) E_{j}(\boldsymbol{r}-\boldsymbol{\rho}, t-\tau) \\
& =\int \mathrm{d}^{3} \rho \wedge \mathrm{d} \tau \hat{\varepsilon}_{i j}(\boldsymbol{\rho}, \tau) \exp \left(-\rho \nabla-\tau \frac{\partial}{\partial t}\right) E_{j}(\boldsymbol{r}, t),
\end{aligned}
$$

where the real-valued tensor function $\hat{\varepsilon}_{i j}=\hat{\varepsilon}_{i j}(\rho, \tau)$ characterizes the material properties and where the integration is written as going over the whole space-time and restrictions of this integration (e.g., to prehistory, causality) are thought to be included by vanishing of this tensor function in certain regions. These restrictions, for example, to the prehistory of the field evolution lead to properties of analyticity and thus to relations between real and imaginary part of the Fourier transform of $\hat{\varepsilon}_{i j}(\rho, \tau)$ which are called Kramers-Kronig relations which we do not discuss here (e.g., [7]). Relation (3.1) means that the most general linear constitutive relations are also nonlocal in space that describes the spatial dispersion ${ }^{4}$. The homogeneity of the medium is expressed by the property that $\hat{\varepsilon}_{i j}(\rho, \tau)$ does not explicitly depend on the considered space-time point $(\boldsymbol{r}, t)$ but only on the differences $(\rho, \tau)$ to this point.

Using the Fourier transform $\varepsilon_{i j}(\boldsymbol{k}, \omega)$ of $\hat{\varepsilon}_{i j}(\rho, \tau)$ according to [9] [10]

$$
\begin{aligned}
& \varepsilon_{i j}(\boldsymbol{k}, \omega)=\int \mathrm{d}^{3} \rho \wedge \mathrm{d} \tau \hat{\varepsilon}_{i j}(\boldsymbol{\rho}, \tau) \mathrm{e}^{-\mathrm{i}(\boldsymbol{k} \rho-\omega \tau)}, \\
& \hat{\varepsilon}_{i j}(\boldsymbol{\rho}, \tau)=\frac{1}{(2 \pi)^{4}} \int \mathrm{d}^{3} k \wedge \mathrm{d} \omega \varepsilon_{i j}(\boldsymbol{k}, \omega) \mathrm{e}^{\mathrm{i}(\boldsymbol{k} \rho-\omega \tau)},
\end{aligned}
$$

the constitutive Equation (3.1) can be represented by

$$
D_{i}(\boldsymbol{r}, t)=\varepsilon_{i j}\left(-\mathrm{i} \nabla, \mathrm{i} \frac{\partial}{\partial t}\right) E_{j}(\boldsymbol{r}, t),
$$

where $\varepsilon_{i j}(\boldsymbol{k}, \omega)$ denotes the complex permittivity tensor. After Fourier transformation of the electric field $\boldsymbol{E}(\boldsymbol{r}, t)$ (analogously $\boldsymbol{D}(\boldsymbol{r}, t)$ and $\boldsymbol{B}(\boldsymbol{r}, t)$ ) ac-

${ }^{4}$ According to Silin and Rukhadse [11] (p.14) the notion "spatial dispersion" was introduced by Gertsenshteyn. Clearly, the name "spatial dispersion" is not analogous to "frequency dispersion" which then has to be better named "temporal dispersion" or vice versa the "spatial dispersion" then "wave-vector dispersion". 
cording to

$$
\begin{aligned}
& \boldsymbol{E}(\boldsymbol{k}, \omega)=\int \mathrm{d}^{3} r \wedge \mathrm{d} t \boldsymbol{E}(\boldsymbol{r}, t) \mathrm{e}^{-\mathrm{i}(\boldsymbol{k r}-\omega t)}, \\
& \boldsymbol{E}(\boldsymbol{r}, t)=\frac{1}{(2 \pi)^{4}} \int \mathrm{d}^{3} k \wedge \mathrm{d} \omega \boldsymbol{E}(\boldsymbol{k}, \omega) \mathrm{e}^{\mathrm{i}(\boldsymbol{k r}-\omega t)},
\end{aligned}
$$

the constitutive relation (3.3) takes on the well-known form (e.g., [7] [8] [9] [10] [11] and others)

$$
D_{i}(\boldsymbol{k}, \omega)=\varepsilon_{i j}(\boldsymbol{k}, \omega) E_{j}(\boldsymbol{k}, \omega) .
$$

The dispersion of the medium is here expressed by the dependence of the permittivity tensor $\varepsilon_{i j}(\boldsymbol{k}, \omega)$ on wave vector $\boldsymbol{k}$ and frequency $\omega$ and the anisotropy by its tensor character.

The electric field $\boldsymbol{E}(\boldsymbol{r}, t)$ and the electric induction $\boldsymbol{D}(\boldsymbol{r}, t)$ are real quantities. From this follows for the Fourier transform of the electric field $\boldsymbol{E}(\boldsymbol{r}, t)$ (analogously $\boldsymbol{D}(\boldsymbol{r}, t)$ and $\boldsymbol{B}(\boldsymbol{r}, t)$ )

$$
\boldsymbol{E}(\boldsymbol{r}, t)=(\boldsymbol{E}(\boldsymbol{r}, t))^{*}, \Leftrightarrow \boldsymbol{E}(\boldsymbol{k}, \omega)=\left(\boldsymbol{E}\left(-\boldsymbol{k}^{*},-\omega^{*}\right)\right)^{*} .
$$

As a consequence, the permittivity tensor $\varepsilon_{i j}(\boldsymbol{k}, \omega)$ possesses the general symmetry property

$$
\varepsilon_{i j}(\boldsymbol{k}, \omega)=\left(\varepsilon_{i j}\left(-\boldsymbol{k}^{*},-\omega^{*}\right)\right)^{*} .
$$

Local or differential conservation laws of energy and momentum can only be derived under the condition that the medium is lossless which means that it does not have any dissipation or accumulation or transmission of energy and momentum to other frequencies and wave vectors. As the later derivations show, the condition for this is the following symmetry

$$
\hat{\varepsilon}_{i j}(\rho, \tau)=\hat{\varepsilon}_{j i}(-\rho,-\tau),
$$

which after Fourier transformation according to (3.2) and in connection with (3.7) takes on the following form

$$
\varepsilon_{i j}(\boldsymbol{k}, \omega)=\varepsilon_{j i}(-\boldsymbol{k},-\omega)=\left(\varepsilon_{j i}\left(\boldsymbol{k}^{*}, \omega^{*}\right)\right)^{*},
$$

and which for dispersive media can only be satisfied approximately for certain regions of wave vector and frequency. Such kind of conditions are closely related to Onsager conditions for quasi-stationary processes [7] (section 21, Ed. 1982) but instead of a rigorous derivation from basic principles we prefer again that one can conclude this from the necessary conditions for the most general possibility of derivation of differential conservation laws of energy and momentum. These conditions should not be confused with the influence of point group symmetries on the medium properties which additionally may be present or may not. The symmetry conditions which are related to different inversion symmetries are discussed in next Section.

To treat spatial dispersion it is mostly appropriate to make a Taylor-series expansion of $\varepsilon_{i j}(\boldsymbol{k}, \omega)$ in powers of the full wave-vector $\boldsymbol{k}$ (i.e., at $\boldsymbol{k}_{0}=\mathbf{0}$ ) 
with notations in [9] (p. 155) and for the inverse tensor $\varepsilon_{i j}^{-1}(\boldsymbol{k}, \omega)$ in the new chapter XII in [7] (Ed. 1982) (Landau and Lifshits prefer there mainly to work with this inverse tensor)

$$
\begin{aligned}
& \varepsilon_{i j}(\boldsymbol{k}, \omega)=\varepsilon_{i j}(\omega)+\mathrm{i} \gamma_{i j k}(\omega) k_{k}+\alpha_{i j k l}(\omega) k_{k} k_{l}+\cdots, \\
& \varepsilon_{i j}^{-1}(\boldsymbol{k}, \omega)=\varepsilon_{i j}^{-1}(\omega)+\mathrm{i} \delta_{i j k}(\omega) k_{k}+\beta_{i j k l}(\omega) k_{k} k_{l}+\cdots,
\end{aligned}
$$

that means for the expansion (3.10)

$$
\varepsilon_{i j}(\omega)=\varepsilon_{j i}(-\omega), \quad \gamma_{i j k}(\omega)=-\gamma_{j i k}(-\omega), \quad \alpha_{i j k l}(\omega)=\alpha_{j i k l}(-\omega), \cdots
$$

The approximate transition from one to the inverse tensor in (3.10) is easily to make.

We mention here shortly that in the most common treatment of macroscopic electrodynamics with two constitutive equations $D_{i}(\boldsymbol{k}, \omega)=\varepsilon_{i j}(\omega) E_{j}(\boldsymbol{k}, \omega)$ and $B_{i}(\boldsymbol{k}, \omega)=\mu_{j i}(\omega) H_{j}(\boldsymbol{k}, \omega)$ the more general tensor $\varepsilon_{i j}(\boldsymbol{k}, \omega)$ corresponds to the special one ( $\varepsilon_{i j k}$ is Levi-Civita pseudo-tensor)

$$
\varepsilon_{i j}(\boldsymbol{k}, \omega)=\varepsilon_{i j}(\omega)+\frac{c^{2}}{\omega^{2}} \varepsilon_{i k m} \varepsilon_{j l n}\left(\delta_{m n}-\mu_{m n}^{-1}(\omega)\right) k_{k} k_{l} .
$$

This shows that a possible magnetization appears here as effect of spatial dispersion of second order in wave-vector $\boldsymbol{k}$ that is important for the energymomentum tensor and also for the boundary conditions at such medium. Furthermore, we see that the tensor $\alpha_{i j k l}(\omega)$ in (3.10) is more general and usually contains more non-vanishing terms than this special tensor proportional to $k_{k} k_{l}$ in $(3.12)^{5}$. Only for magnetostatics this concept not used in present article is less appropriate.

\section{Additional Restrictions of Tensor $\varepsilon_{i j}(k, \omega)$ for Discrete Symmetries of Spatial and Time Inversion}

We now consider the most simple discrete symmetries of order 2 .

1) Spatial inversion (presence of symmetry center)

The presence of spatial inversion that means invariance of the medium with respect to the transformation $\boldsymbol{r} \rightarrow-\boldsymbol{r}$ of the coordinates where due to the homogeneity the chosen coordinate origin is arbitrary and due to the property of $\boldsymbol{E}$ and $\boldsymbol{D}$ to be genuine vectors changing their sign under this transformation (in contrast, $\boldsymbol{B}$ is a pseudo-vector) leads to

$$
\varepsilon_{i j}(\boldsymbol{k}, \omega)=\varepsilon_{i j}(-\boldsymbol{k}, \omega)=\left(\varepsilon_{i j}\left(\boldsymbol{k}^{*},-\omega^{*}\right)\right)^{*},
$$

where in the second step (3.7) was used in addition. The first part of this condition means that for media with spatial inversion the components of the permittivity tensor $\varepsilon_{i j}(\boldsymbol{k}, \omega)$ are mutually independent from each other and are even functions of the wave vector $\boldsymbol{k}$, whereas it does not mean a restriction for its dependence on the frequency $\omega$. In composition with the condition (3.9) for absence of dissipation we have

${ }^{5}$ For a deeper understanding we recommend here again the very instructive section 79 in [7] (Ed. 1982). 


$$
\varepsilon_{i j}(\boldsymbol{k}, \omega)=\varepsilon_{i j}(-\boldsymbol{k}, \omega)=\varepsilon_{j i}(\boldsymbol{k},-\omega)=\varepsilon_{j i}(-\boldsymbol{k}, \omega),
$$

which relates different components of the permittivity tensor and is not true for regions of $\boldsymbol{k}$ and $\omega$ where dissipation is not negligible. If we deal with spatial dispersion by expansion of $\varepsilon_{i j}(\boldsymbol{k}, \omega)$ in powers of the wave vector $\boldsymbol{k}$ then we can use mainly the first part $\varepsilon_{i j}(\boldsymbol{k}, \omega)=\varepsilon_{i j}(-\boldsymbol{k}, \omega)$ of these symmetry conditions which are true also in case of dissipation.

2) Time inversion (nonmagnetic symmetry classes)

The presence of time inversion that means of invariance of the medium with respect to the transformation $t \rightarrow-t$ taking into account that $\boldsymbol{E}$ and $\boldsymbol{D}$ do not change their sign under this transformation (in contrast, $\boldsymbol{B}$ changes it) leads to

$$
\varepsilon_{i j}(\boldsymbol{k}, \omega)=\varepsilon_{i j}(\boldsymbol{k},-\omega)=\left(\varepsilon_{i j}\left(-\boldsymbol{k}^{*}, \omega^{*}\right)\right)^{*},
$$

where in addition (3.7) is used in last equality. The first part of this condition means that for media with spatial inversion the components of the permittivity tensor $\varepsilon_{i j}(\boldsymbol{k}, \omega)$ are mutually independent from each other even functions of the frequency $\omega$, whereas it does not mean a restriction for its dependence on the wave vector $\boldsymbol{k}$. In composition with the condition (3.9) for absence of dissipation we have here

$$
\varepsilon_{i j}(\boldsymbol{k}, \omega)=\varepsilon_{i j}(\boldsymbol{k},-\omega)=\varepsilon_{j i}(-\boldsymbol{k}, \omega)=\varepsilon_{j i}(-\boldsymbol{k},-\omega) .
$$

In expansions of the permittivity tensor $\varepsilon_{i j}(k, \omega)$ in powers of $\boldsymbol{k}$ one may use here mainly the part $\varepsilon_{i j}(\boldsymbol{k}, \omega)=\varepsilon_{j i}(-\boldsymbol{k}, \omega)$ for simplifications which, however, are true only under neglect of dissipation.

3) Product of spatial inversion with time inversion (nongyrotropic media)

The presence of the product of spatial inversion with time inversion (including, evidently, the case of presence of both symmetry elements separately and therefore also of their product) leads to the symmetry

$$
\varepsilon_{i j}(\boldsymbol{k}, \omega)=\varepsilon_{i j}(-\boldsymbol{k},-\omega)=\left(\varepsilon_{i j}\left(\boldsymbol{k}^{*}, \omega^{*}\right)\right)^{*},
$$

where again the condition (3.7) is used in last equality. In composition with the condition for absent dissipation (3.9) we find

$$
\varepsilon_{i j}(\boldsymbol{k}, \omega)=\varepsilon_{i j}(-\boldsymbol{k},-\omega)=\varepsilon_{j i}(-\boldsymbol{k},-\omega)=\varepsilon_{j i}(\boldsymbol{k}, \omega) .
$$

The most interesting part of this relation $\varepsilon_{i j}(\boldsymbol{k}, \omega)=\varepsilon_{j i}(\boldsymbol{k}, \omega)$ describes complete symmetry of the permittivity tensor $\varepsilon_{i j}(\boldsymbol{k}, \omega)$ and this symmetry, by definition, is called non-gyrotropy of a medium and is connected with the symmetry element of product of spatial inversion with time inversion of the medium but not necessarily with both symmetries separately. Clearly, a non-gyrotropic medium possesses this symmetry only in regions of wave vector and frequency where dissipation which is not included in the symmetric part can be neglected. On the other side, gyrotropic media are such media which do not possess this symmetry of the permittivity tensor $\varepsilon_{i j}(\boldsymbol{k}, \omega)$.

Usually, if nothing is said in crystal optics it is meant that the medium pos- 
sesses time inversion as symmetry element in addition to the spatial symmetry elements of one of the considered 32 crystal classes which are then called non-magnetic crystal classes and for which (4.3) is true. From the 32 crystal classes 11 do not possess a symmetry center and 11 possess it and therefore the spatial inversion as symmetry element and thus are non-gyrotropic and (4.1) and (4.5) are true for them in addition. There are 90 magnetic crystal classes from which 32 are trivial ones and correspond to the usual crystal classes but without time inversion as symmetry element.

Furthermore, there exist 58 magnetic crystal classes which contain time inversion not directly as symmetry element but in the form of the product of time inversion with the elements of a coset to an invariant subgroup of one of the 11 groups with only rotations [7] [41]. The 90 magnetic classes form the basis for the symmetry classification of ferromagnetics and anti-ferromagnetics. This concerns natural absence of time inversion as symmetry element but this absence can be generated also artificially under the influence of the medium by an external magnetic field from a primarily non-magnetic class. In the same way, among the 122 crystal classes (magnetic and non-magnetic ones) there are 32 classes with symmetry center for which (4.1) is true and 90 without symmetry center. This is contained in a compact form in Figure 1 copied from our paper [41].

\section{Elimination of Magnetic Field and Three-Dimensional Operator Equation with Relativistic Covariance for the Electric Field}

By differentiation of the second vectorial equation in (2.7) with respect to time and using the first vectorial equation, the magnetic field can be eliminated and using the constitutive Equation (3.3) we obtain the following equation for the electric field

$$
\begin{aligned}
0 & =\left(\nabla_{i} \nabla_{j}-\nabla^{2} \delta_{i j}\right) E_{j}(\boldsymbol{r}, t)+\frac{1}{c^{2}} \frac{\partial^{2}}{\partial t^{2}} D_{i}(\boldsymbol{r}, t) \\
& =\left\{\nabla_{i} \nabla_{j}-\nabla^{2} \delta_{i j}+\frac{1}{c^{2}} \frac{\partial^{2}}{\partial t^{2}} \varepsilon_{i j}\left(-\mathrm{i} \nabla, \mathrm{i} \frac{\partial}{\partial t}\right)\right\} E_{j}(\boldsymbol{r}, t) .
\end{aligned}
$$

This equation for the electric field contains the full information about the electromagnetic field in the medium with exception of some static cases which have to be considered as limiting cases.

Since Equation (5.1) carries the full information about the electromagnetic field the conservation theorems may be derived from it that possesses considerable advantages, in particular, taking into account the dispersion as we will demonstrate this in the following. For such derivations, roughly speaking, we have to multiply this equation from the left with other electric fields $E_{i}^{\prime}(\boldsymbol{r}, t)$. However, in this way we do not get expressions which are relativistic-covariant (they multiply by factors under Lorentz transformations). This shortage can be removed if we divide this equation by $\frac{1}{c^{2}} \frac{\partial^{2}}{\partial t^{2}}$ as will be shown in Appendix A that makes 


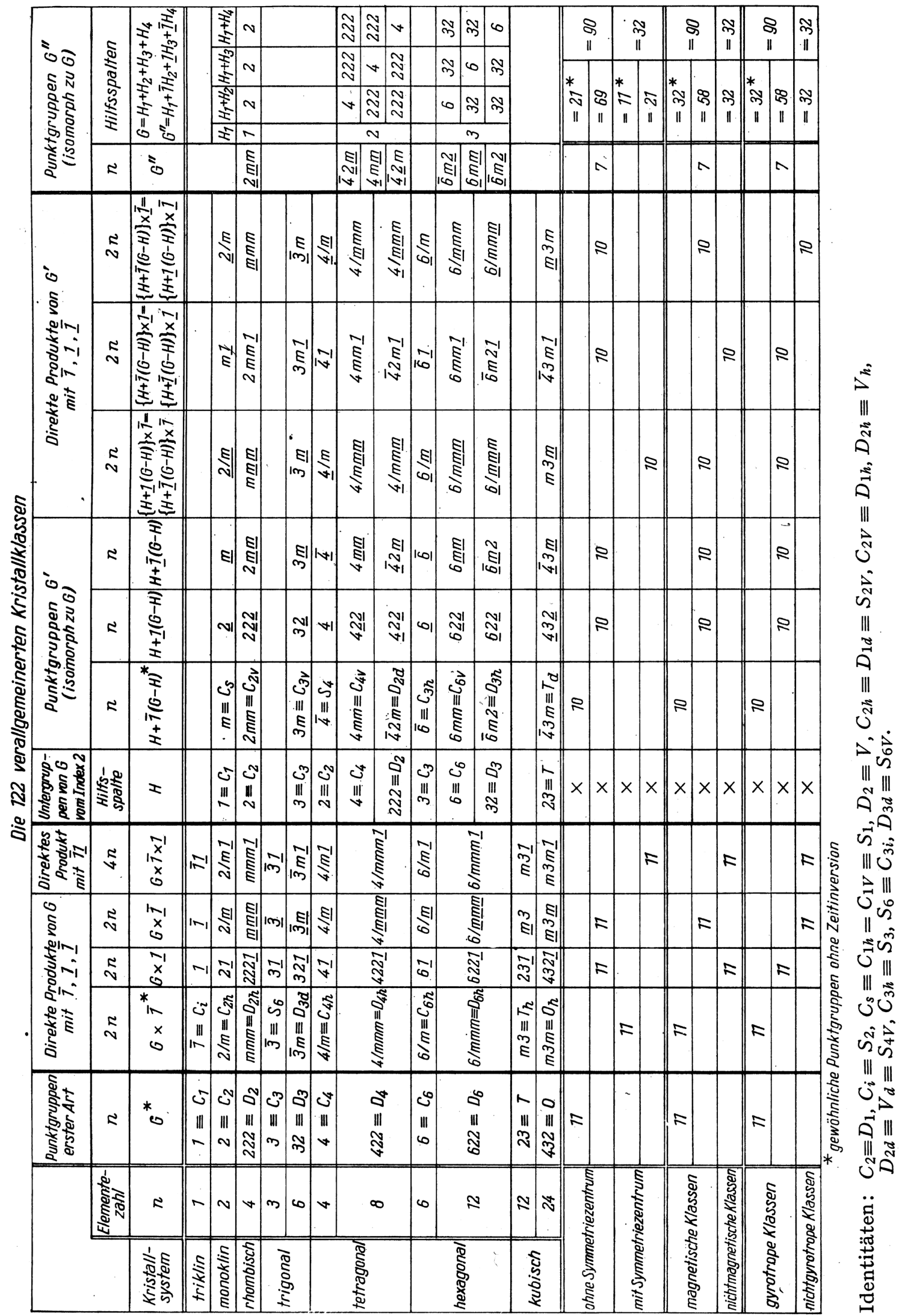

Figure 1. The 122 magnetic and non-magnetic crystal classes (from [41]). Hilfsspalte = auxiliary column. 
difficulties only in the limiting transition to vanishing frequencies. Thus starting from Maxwell equations and constitutive equations as intermediate step (5.1) we arrive at the following vectorial equation for the electric field

$$
L_{i j}\left(-\mathrm{i} \nabla, \mathrm{i} \frac{\partial}{\partial t}\right) E_{j}(\boldsymbol{r}, t)=0
$$

where the tensor operator $L_{i j}\left(-\mathrm{i} \nabla, \mathrm{i} \frac{\partial}{\partial t}\right)$ is defined in the following way

$$
L_{i j}\left(-\mathrm{i} \nabla, \mathrm{i} \frac{\partial}{\partial t}\right) \equiv \frac{c^{2}\left(\nabla_{i} \nabla_{j}-\nabla^{2} \delta_{i j}\right)}{\frac{\partial^{2}}{\partial t^{2}}}+\varepsilon_{i j}\left(-\mathrm{i} \nabla, \mathrm{i} \frac{\partial}{\partial t}\right) .
$$

The vectorial equation for the electric field (5.2) together with definition (5.3) forms a closed system of equations of macroscopic electrodynamics of homogeneous media and, moreover, is relativistic-covariant (contrary to (5.1)) and are appropriate for the derivation of the energy-momentum tensor. After Fourier transformation of the electric field according to (3.4) we obtain from (5.2) the equation for the Fourier components $\boldsymbol{E}(\boldsymbol{k}, \omega)$ of the electric field and then the magnetic field $\boldsymbol{B}(\boldsymbol{k}, \omega)$

$$
L_{i j}(\boldsymbol{k}, \omega) E_{j}(\boldsymbol{k}, \omega)=0, \quad B_{i}(\boldsymbol{k}, \omega)=\frac{c}{\omega} \varepsilon_{i j k} k_{j} E_{k}(\boldsymbol{k}, \omega),
$$

with the tensor operator $L_{i j}(\boldsymbol{k}, \omega)$ in this equation defined by

$$
\begin{aligned}
L_{i j}(\boldsymbol{k}, \omega) & \equiv \frac{c^{2}\left(k_{i} k_{j}-\boldsymbol{k}^{2} \delta_{i j}\right)}{\omega^{2}}+\varepsilon_{i j}(\boldsymbol{k}, \omega) \\
& =\frac{c^{2}}{\omega^{2}}\left\{k_{i} k_{j}-\left(\boldsymbol{k}^{2}-\frac{\omega^{2}}{c^{2}}\right) \delta_{i j}\right\}+4 \pi \chi_{i j}(\boldsymbol{k}, \omega),
\end{aligned}
$$

where $\chi_{i j}(\boldsymbol{k}, \omega) \equiv \frac{\varepsilon_{i j}(\boldsymbol{k}, \omega)-\delta_{i j}}{4 \pi}$ is the general susceptibility tensor. Since the tensor $\chi_{i j}(\boldsymbol{k}, \omega)$ may be a complicated function of the wave-vector $\boldsymbol{k}$ and, in particular, of the frequency $\omega$ it is hardly possible to write down a Lagrange function for the system and the usual formalism of derivation of the energy-momentum tensor from such function is almost impossible.

Equations (5.4) with operator (5.5) as transformed Equation (5.2) possess also a relativistically covariant form in three-dimensional orthogonal coordinates for arbitrary inertial systems $\mathcal{I}^{\prime}$

$$
L_{i j}^{\prime}\left(\boldsymbol{k}^{\prime}, \omega^{\prime}\right) E_{j}^{\prime}\left(\boldsymbol{k}^{\prime}, \omega^{\prime}\right)=0,
$$

with

$$
L_{i j}^{\prime}\left(\boldsymbol{k}^{\prime}, \omega^{\prime}\right) \equiv \frac{c^{2}\left(k_{i}^{\prime} k_{j}^{\prime}-\boldsymbol{k}^{\prime 2} \delta_{i j}\right)}{\omega^{\prime 2}}+\varepsilon_{i j}^{\prime}\left(\boldsymbol{k}^{\prime}, \omega^{\prime}\right)
$$

where $\varepsilon_{i j}^{\prime}\left(\boldsymbol{k}^{\prime}, \omega^{\prime}\right)$ is related to $\varepsilon_{i j}(\boldsymbol{k}, \omega)$ by a transformation which we derive in detail in Appendix A. For an inertial system $\mathcal{I}^{\prime}$ moving with velocity $\boldsymbol{V}$ in the inertial system $\mathcal{I}$ according to the special Lorentz transformation (A.14) it 
possesses the form (A.20) [42]

$$
\begin{aligned}
\underbrace{\varepsilon_{i j}^{\prime}\left(\boldsymbol{k}^{\prime}, \omega^{\prime}\right)-\delta_{i j}=}_{=4 \pi \chi_{i j}^{\prime}\left(\boldsymbol{k}^{\prime}, \omega^{\prime}\right)} & \left\{\frac{V_{i} V_{k}}{\boldsymbol{V}^{2}}+\gamma\left(\delta_{i k}-\frac{V_{i} V_{k}}{\boldsymbol{V}^{2}}+\frac{\boldsymbol{k}^{\prime} \boldsymbol{V} \delta_{i k}-V_{i} k_{k}^{\prime}}{\omega^{\prime}}\right)\right\} \\
& \cdot\left\{\frac{V_{j} V_{l}}{\boldsymbol{V}^{2}}+\gamma\left(\delta_{j l}-\frac{V_{j} V_{l}}{\boldsymbol{V}^{2}}+\frac{\boldsymbol{k}^{\prime} \boldsymbol{V} \delta_{j l}-V_{j} k_{l}^{\prime}}{\omega^{\prime}}\right)\right\} \underbrace{\left(\varepsilon_{k l}(\boldsymbol{k}, \omega)-\delta_{k l}\right)}_{=4 \pi \chi_{i j}(\boldsymbol{k}, \omega)},
\end{aligned}
$$

with the following relations between $\left(\boldsymbol{k}^{\prime}, \omega^{\prime}\right)$ and $(\boldsymbol{k}, \omega)$ and their inversion by $\boldsymbol{V} \rightarrow-\boldsymbol{V}$ (see (A.15)

$$
\begin{aligned}
& \boldsymbol{k}^{\prime}=\boldsymbol{k}+\left((\gamma-1) \frac{\boldsymbol{k} \boldsymbol{V}}{\boldsymbol{V}^{2}}-\gamma \frac{\omega}{c^{2}}\right) \boldsymbol{V}, \quad \omega^{\prime}=\gamma(\omega-\boldsymbol{k} \boldsymbol{V}), \\
& \boldsymbol{k}=\boldsymbol{k}^{\prime}+\left((\gamma-1) \frac{\boldsymbol{k}^{\prime} \boldsymbol{V}}{\boldsymbol{V}^{2}}+\gamma \frac{\omega^{\prime}}{c^{2}}\right) \boldsymbol{V}, \quad \omega=\gamma\left(\omega^{\prime}+\boldsymbol{k}^{\prime} \boldsymbol{V}\right),
\end{aligned}
$$

and with relativistic invariant

$$
\boldsymbol{k}^{\prime 2}-\frac{\omega^{\prime 2}}{c^{2}}=\boldsymbol{k}^{2}-\frac{\omega^{2}}{c^{2}}
$$

In $\varepsilon_{k l}(\boldsymbol{k}, \omega)$ on the right-hand side of (5.8) one has yet to express the arguments $(\boldsymbol{k}, \omega)$ by the arguments $\left(\boldsymbol{k}^{\prime}, \omega^{\prime}\right)$ according to the transformation formulae (5.9) to have the same variables on both sides. This means that the transformed permittivity tensor $\varepsilon_{i j}^{\prime}\left(\boldsymbol{k}^{\prime}, \omega^{\prime}\right)$ becomes dependent on the wave vector $\boldsymbol{k}^{\prime}$ even in case that the primary permittivity tensor $\varepsilon_{i j}(\boldsymbol{k}, \omega)$ in the resting system of the medium does not depend on the wave vector $\boldsymbol{k}$. However, this dependence on the wave vector $\boldsymbol{k}$ in the system moving with velocity $\boldsymbol{V}$ which formally means spatial dispersion of the medium is of some other kind than the natural dependence of the permittivity of a medium on wave vector $\boldsymbol{k}$ in resting system and it is not reasonable to expand it in a Taylor series in $\boldsymbol{k}$. For the formal derivation of the energy-momentum tensor these differences are not of importance.

The transformation formulae (5.8) for the permittivity tensor from one to another inertial system and for (5.9) simplify essentially in non-relativistic approximation $\frac{|\boldsymbol{V}|}{c} \ll 1$ if we neglect quadratic and higher terms in $\frac{\boldsymbol{V}}{c}$ in comparison to linear terms in $\frac{V}{c}$ (e.g., $\left.\gamma \rightarrow 1\right)$ that we do not write down. From transformations (5.8) together with (5.9) we see that if we change at the same time the signs of $\boldsymbol{k}$ and $\omega$ this also changes at the same time the signs of $\boldsymbol{k}^{\prime}$ and $\omega^{\prime}$ according to

$$
(\boldsymbol{k}, \omega) \rightarrow(-\boldsymbol{k},-\omega), \Leftrightarrow\left(\boldsymbol{k}^{\prime}, \omega^{\prime}\right) \rightarrow\left(-\boldsymbol{k}^{\prime},-\omega^{\prime}\right) .
$$

As expected this means that the condition (3.9) for the dissipation-free case transforms into a corresponding condition for the dissipation-free case in an arbitrary inertial system $\mathcal{I}^{\prime}$ moving with velocity $\boldsymbol{V}$ in inertial system $\mathcal{I}$

$$
\varepsilon_{i j}(\boldsymbol{k}, \omega)=\varepsilon_{j i}(-\boldsymbol{k},-\omega), \Leftrightarrow \varepsilon_{i j}^{\prime}\left(\boldsymbol{k}^{\prime}, \omega^{\prime}\right)=\varepsilon_{j i}^{\prime}\left(-\boldsymbol{k}^{\prime},-\omega^{\prime}\right),
$$


and is therefore invariant with respect to Lorentz transformations as one could have to expect for such a physical property.

It is seen that the condition (3.9) for absent losses can be continued to the following condition for $L_{i j}(\boldsymbol{k}, \omega)$

$$
L_{i j}(\boldsymbol{k}, \omega)=L_{j i}(-\boldsymbol{k},-\omega)=\left(L_{j i}\left(\boldsymbol{k}^{*}, \omega^{*}\right)\right)^{*} .
$$

Therefore, if (5.13) is satisfied we can write down in addition to (5.2) the following equation for the electric field

$$
L_{i j}\left(\mathrm{i} \nabla,-\mathrm{i} \frac{\partial}{\partial t}\right) E_{i}(\boldsymbol{r}, t)=0 .
$$

The two Equations (5.2) and (5.14) form the basis of our derivations of local conservation laws and it possesses a great advantage that we have only one field function for the electric field in these equations in comparison to the electric and magnetic field in the common derivations.

We now consider quasiplane and quasimonochromatic waves in the form

$$
\boldsymbol{E}(\boldsymbol{r}, t)=\boldsymbol{E}_{0}(\boldsymbol{r}, t) \mathrm{e}^{\mathrm{i}\left(\boldsymbol{k}_{0} r-\omega_{0} t\right)}+\boldsymbol{E}_{0}^{*}(\boldsymbol{r}, t) \mathrm{e}^{-\mathrm{i}\left(\boldsymbol{k}_{0} r-\omega_{0} t\right)}, \quad\left(\boldsymbol{k}_{0}=\boldsymbol{k}_{0}^{*}, \omega_{0}=\omega_{0}^{*}\right),
$$

where $\boldsymbol{E}_{0}(\boldsymbol{r}, t)$ is a slowly varying complex vectorial amplitude and $\boldsymbol{k}_{0}$ a mean wave vector and $\omega_{0}$ a mean frequency. We suppose that $\boldsymbol{k}_{0}$ and $\omega_{0}$ are real and exclude in such way, but only for simplicity, evanescent waves with complex values of these quantities which may exist even in lossless media (for example, waves under total reflection in the lossless optically thinner medium or surface waves). The inclusion of such waves would complicate the following considerations but does not destroy the existence of local conservation theorems.

The approximations which we make in the following are that due to slowness of changing of the amplitudes $\boldsymbol{E}_{0}(\boldsymbol{r}, t)$ in such way that we may take into account in expansions only a small number of spatial and temporal derivatives of these amplitudes. This means that the wave vectors and frequencies in the Fourier decomposition of the quasiplane and quasimonochromatic wave are concentrated around $\boldsymbol{k}_{0}$ and $\omega_{0}$ (and, clearly, around $-\boldsymbol{k}_{0}$ and $-\omega_{0}$ ) and the two complex conjugated parts in (5.15) are well separated. The supposition and at once approximation in the following is that we can deal with both parts as independent solutions of the wave equation for the electric field. This is apparently equivalent to some averaging procedure over terms with rapidly varying frequencies and wave vectors which then vanish from the equations such as made in [7] and is justified for quasiplane and quasimonochromatic waves.

If we insert the first part from the right-hand side of (5.15) as independent solution into Equation (5.2) we obtain the following equation for the slowly varying complex amplitude $\boldsymbol{E}_{0}(\boldsymbol{r}, t)$

$$
\begin{aligned}
0 & =L_{i j}\left(\boldsymbol{k}_{0}-\mathrm{i} \nabla, \omega_{0}+\mathrm{i} \frac{\partial}{\partial t}\right) E_{0, j}(\boldsymbol{r}, t) \\
& =\left\{\left(L_{i j}\right)_{0}-\mathrm{i}\left(\frac{\partial L_{i j}}{\partial k_{l}}\right)_{0} \nabla_{l}+\mathrm{i}\left(\frac{\partial L_{i j}}{\partial \omega}\right)_{0} \frac{\partial}{\partial t}\right.
\end{aligned}
$$




$$
\left.-\frac{1}{2}\left(\left(\frac{\partial^{2} L_{i j}}{\partial k_{l} \partial k_{m}}\right)_{0} \nabla_{l} \nabla_{m}-2\left(\frac{\partial^{2} L_{i j}}{\partial k_{l} \partial \omega}\right)_{0} \nabla_{l} \frac{\partial}{\partial t}+\left(\frac{\partial^{2} L_{i j}}{\partial \omega^{2}}\right)_{0} \frac{\partial^{2}}{\partial t^{2}}\right)+\cdots\right\} E_{0, j}(\boldsymbol{r}, t),
$$

where index " 0 " means that the corresponding derivatives have to be taken for $\boldsymbol{k}=\boldsymbol{k}_{0}$ and $\omega=\omega_{0}$ (e.g., $\left.\left(L_{i j}\right)_{0} \equiv L_{i j}\left(\boldsymbol{k}_{0}, \omega_{0}\right)\right)$. In the following, we take the derivatives of the slowly varying amplitudes up to the second order but before this we introduce a shorter relativistic-covariant notation of the equations for $\boldsymbol{E}_{0}(\boldsymbol{r}, t)$ and $\boldsymbol{E}_{0}^{*}(\boldsymbol{r}, t)$.

Our derivation of the energy-momentum tensor is similar to the derivation of approximate equations for beam solutions with the only difference that in last case the determinant of $L_{i j}$ has to be taken as starting point for the expansion to get the equation for the main component of $\boldsymbol{E}_{0}(\boldsymbol{r}, t)$.

\section{Local Action Conservation in Relativistic Covariant Form}

The derivation of local (or differential) laws of action conservation and of other local conservation theorems becomes much more concise if we introduce for abbreviation the following four-dimensional notations of special theory of relativity $^{6}$

$$
\begin{aligned}
& r \equiv(\boldsymbol{r}, \mathrm{i} c t), \quad k \equiv\left(\boldsymbol{k}, \frac{\mathrm{i} \omega}{c}\right), \quad r^{2}=\boldsymbol{r}^{2}-c^{2} t^{2}, \quad k^{2}=\boldsymbol{k}^{2}-\frac{\omega^{2}}{c^{2}}, \quad \nabla \equiv\left(\nabla, \frac{1}{\mathrm{i} c} \frac{\partial}{\partial t}\right), \\
& -\mathrm{i} \nabla \mathrm{e}^{\mathrm{i} k_{0} r}=-\mathrm{i}\left(\nabla, \frac{1}{\mathrm{i} c} \frac{\partial}{\partial t}\right) \mathrm{e}^{\mathrm{i}\left(\boldsymbol{k}_{0} r-\omega_{0} t\right)}=\mathrm{e}^{\mathrm{i}\left(\boldsymbol{k}_{0} r-\omega_{0} t\right)}\left(\boldsymbol{k}_{0}-\mathrm{i} \nabla, \frac{\mathrm{i}}{c}\left(\omega_{0}+\mathrm{i} \frac{\partial}{\partial t}\right)\right) .
\end{aligned}
$$

An advantage of the four-dimensional formalism is that we obtain the results in relativistic covariant form.

We may write the equations for the electric field (5.2) and (5.14) in the concise form ${ }^{7}$

$$
L_{i j}(-\mathrm{i} \nabla) E_{j}(r)=0, \quad L_{i j}(\mathrm{i} \nabla) E_{i}(r)=0 .
$$

with $L_{i j}(k)$ in relativistic-covariant form (see Appendix A, (25))

${ }^{6}$ Modern development mainly for preparing the transition to General relativity theory favors to use only representations by real components for space-time. This makes it necessary to distinguish between contravariant and covariant components of vectors and tensors but this becomes very inconvenient for our purposes. According to Pauli [5] (Part III, p. 71), the historically older notation $\left(x^{4}=\right) x_{4}=\mathrm{i} c t$ was first used by Poincaré in 1906 in Journal "R.C. Circ. mat. Palermo 21, 129" for vectors $x=(\boldsymbol{x} \equiv(x, y, z), t)$ of space-time later called Minkowski space (after Minkowski's Lecture "Raum und Zeit" in Köln in 1908, published in 1909 [43] (republ. [44, 36]) in the year of his premature death). Apart from basic Maxwell equations, Einstein up to 1912 preferred to write down all four-dimensional vectorial relations separately in the 4 components and mentions $x_{4}=\mathrm{ict}$ only shortly in 1910 with reference to Minkowski [43] that can be traced from the collection of Einstein's scientific papers [1] (p. 138, for corresponding article). Minkowski on his part finds it in [43] to be a "very pregnant manner" to cloth $s=\sqrt{-1} c t$ in the "mystic formula" $3 \times 10^{5} \mathrm{~km}=\sqrt{-1} \mathrm{secs}$.

${ }^{7}$ Deviating from (6.1) we now used in the notations of the argument of the electric field $E_{j}$ and of the operator function $L_{i j}$ the four-dimensional notations in the form $r=(\boldsymbol{r}, t)$ and $k=(\boldsymbol{k}, \omega)$ which we do not want to change here into that of (6.1). However, by comparison with second line in (1) it seems that this does not cause problems. 


$$
L_{i j}(k) \equiv L_{i j}(\boldsymbol{k}, \omega)=\frac{c^{2}}{\omega^{2}}\left(k_{i} k_{j}-\boldsymbol{k}^{2} \delta_{i j}\right)+\varepsilon_{i j}(\boldsymbol{k}, \omega),
$$

and $k$ substituted by $-\mathrm{i} \nabla$. The quasiplane and quasimonochromatic waves (5.15) take on the shorter form

$$
E_{j}(r)=E_{0, j}(r) \mathrm{e}^{\mathrm{i} k_{0} r}+E_{0, j}^{*}(r) \mathrm{e}^{-\mathrm{i} k_{0} r} .
$$

In the same approximation as in (5.16) we obtain from the first of Equation (6.2)

$$
\begin{aligned}
0 & =L_{i j}\left(k_{0}-\mathrm{i} \nabla\right) E_{0, j}(r) \\
& =\left\{\left(L_{i j}\right)_{0}-\mathrm{i}\left(\frac{\partial L_{i j}}{\partial k_{\lambda}}\right)_{0} \nabla_{\lambda}-\frac{1}{2 !}\left(\frac{\partial^{2} L_{i j}}{\partial k_{\lambda} \partial k_{\mu}}\right)_{0} \nabla_{\lambda} \nabla_{\mu}+\cdots\right\} E_{0, j}(r),
\end{aligned}
$$

and from the second equation

$$
\begin{aligned}
0 & =L_{i j}\left(k_{0}+\mathrm{i} \nabla\right) E_{0, i}^{*}(r) \\
& =\left\{\left(L_{i j}\right)_{0}+\mathrm{i}\left(\frac{\partial L_{i j}}{\partial k_{\lambda}}\right)_{0} \nabla_{\lambda}-\frac{1}{2 !}\left(\frac{\partial^{2} L_{i j}}{\partial k_{\lambda} \partial k_{\mu}}\right)_{0} \nabla_{\lambda} \nabla_{\mu}+\cdots\right\} E_{0, i}^{*}(r) .
\end{aligned}
$$

We first derive a conservation theorem which is even more fundamental than the theorem for energy-momentum conservation since it may be extended to inhomogeneous media.

If we multiply (6.5) by $E_{0, i}^{*}(r)$ and (6.6) by $E_{0, j}(r)$ and form the difference of the obtained equations then it can be written as the 4-divergence of a 4 -vector function in the following way

$$
\begin{aligned}
0= & -\mathrm{i}\left\{E_{0, i}^{*}(r) L_{i j}\left(k_{0}-\mathrm{i} \nabla\right) E_{0, j}(r)-E_{0, j}(r) L_{i j}\left(k_{0}+\mathrm{i} \nabla\right) E_{0, i}^{*}(r)\right\} \\
= & \nabla_{\lambda}\left\{-\left(\frac{\partial L_{i j}}{\partial k_{\lambda}}\right)_{0} E_{0, i}^{*}(r) E_{0, j}(r)\right. \\
& \left.+\frac{\mathrm{i}}{2}\left(\frac{\partial^{2} L_{i j}}{\partial k_{\lambda} \partial k_{\mu}}\right)_{0}\left(E_{0, i}^{*}(r) \nabla_{\mu} E_{0, j}(r)-E_{0, j}(r) \nabla_{\mu} E_{0, i}^{*}(r)\right)+\cdots\right\} .
\end{aligned}
$$

The terms are explicitly written down up to first-order derivatives of the slowly varying amplitudes but the higher-order terms on the right-hand side can also be represented as 4-divergence of a 4 -vector $T_{\lambda}(r)$ and (6.7) possess the form of a vanishing 4-divergence and can be included in the local conservation law

$$
\nabla_{\lambda} T_{\lambda}(r)=0,
$$

with $T_{\lambda}(r)$ defined by (remind: $\alpha \equiv 4 \pi$ in CGS or Gauss system)

$$
\begin{aligned}
\alpha T_{\lambda}(r)= & -\left(\frac{\partial L_{i j}}{\partial k_{\lambda}}\right)_{0} E_{0, i}^{*}(r) E_{0, j}(r) \\
& +\frac{\mathrm{i}}{2}\left(\frac{\partial^{2} L_{i j}}{\partial k_{\lambda} \partial k_{\mu}}\right)_{0}\left(E_{0, i}^{*}(r) \nabla_{\mu} E_{0, j}(r)-E_{0, j}(r) \nabla_{\mu} E_{0, i}^{*}(r)\right)+\cdots
\end{aligned}
$$

In three-dimensional separation according to the definition 


$$
T_{\lambda}(r)=\left(T_{l}(\boldsymbol{r}, t), \mathrm{i} c s(\boldsymbol{r}, t)\right),
$$

the local conservation theorem (6.8) takes on the form

$$
\nabla_{l} T_{l}(\boldsymbol{r}, t)+\frac{\partial}{\partial t} s(\boldsymbol{r}, t)=0 .
$$

From their dimensions, $s(\boldsymbol{r}, t)$ can be identified with the action density and $T_{l}(\boldsymbol{r}, t)$ with the vector field of action flow density.

From (6.10) and (6.9) we find in three-dimensional representation up to explicitly given first-order derivatives of the slowly varying amplitudes $\boldsymbol{E}_{0}(\boldsymbol{r}, t)$ of the electric field which last take into account the diffraction of beams

$$
\begin{aligned}
\alpha T_{l}(\boldsymbol{r}, t)= & -\left(\frac{\partial L_{i j}}{\partial k_{l}}\right)_{0} E_{0, i}^{*}(\boldsymbol{r}, t) E_{0, j}(\boldsymbol{r}, t) \\
& +\frac{\mathrm{i}}{2}\left(\frac{\partial^{2} L_{i j}}{\partial k_{l} \partial k_{m}}\right)_{0}\left(E_{0, i}^{*}(\boldsymbol{r}, t) \nabla_{m} E_{0, j}(\boldsymbol{r}, t)-E_{0, j}(\boldsymbol{r}, t) \nabla_{m} E_{0, i}^{*}(\boldsymbol{r}, t)\right) \\
& -\frac{\mathrm{i}}{2}\left(\frac{\partial^{2} L_{i j}}{\partial k_{l} \partial \omega}\right)_{0}\left(E_{0, i}^{*}(\boldsymbol{r}, t) \frac{\partial}{\partial t} E_{0, j}(\boldsymbol{r}, t)-E_{0, j}(\boldsymbol{r}, t) \frac{\partial}{\partial t} E_{0, i}^{*}(\boldsymbol{r}, t)\right)+\cdots, \\
\alpha S(\boldsymbol{r}, t)= & \left.\frac{\partial L_{i j}}{\partial \omega}\right)_{0} E_{0, i}^{*}(\boldsymbol{r}, t) E_{0, j}(\boldsymbol{r}, t) \\
& -\frac{\mathrm{i}}{2}\left(\frac{\partial^{2} L_{i j}}{\partial \omega \partial k_{m}}\right)_{0}\left(E_{0, i}^{*}(\boldsymbol{r}, t) \nabla_{m} E_{0, j}(\boldsymbol{r}, t)-E_{0, j}(\boldsymbol{r}, t) \nabla_{m} E_{0, i}^{*}(\boldsymbol{r}, t)\right) \\
+ & \frac{\mathrm{i}}{2}\left(\frac{\partial^{2} L_{i j}}{\partial \omega^{2}}\right)_{0}\left(E_{0, i}^{*}(\boldsymbol{r}, t) \frac{\partial}{\partial t} E_{0, j}(\boldsymbol{r}, t)-E_{0, j}(\boldsymbol{r}, t) \frac{\partial}{\partial t} E_{0, i}^{*}(\boldsymbol{r}, t)\right)+\cdots .
\end{aligned}
$$

Before discussing these expressions we derive the local form of energymomentum conservation.

\section{Local Energy and Momentum Conservation in Relativistic Covariant Form}

In analogy to (6.7) we consider the following combination which can be represented as the 4-divergence of a second-rank 4-tensor

$$
\begin{aligned}
0= & -\mathrm{i}\left\{E_{0, i}^{*}(r)\left(k_{0, \kappa}-\mathrm{i} \nabla_{\kappa}\right) L_{i j}\left(k_{0}-\mathrm{i} \nabla\right) E_{0, j}(r)\right. \\
& \left.-E_{0, j}(r)\left(k_{0, \kappa}+\mathrm{i} \nabla_{\kappa}\right) L_{i j}\left(k_{0}+\mathrm{i} \nabla\right) E_{0, i}^{*}(r)\right\} \\
= & \nabla_{\lambda}\left\{-\left(\frac{\partial\left(k_{\kappa} L_{i j}\right)}{\partial k_{\lambda}}\right)_{0} E_{0, i}^{*}(r) E_{0, j}(r)\right. \\
& \left.+\frac{\mathrm{i}}{2}\left(\frac{\partial^{2}\left(k_{\kappa} L_{i j}\right)}{\partial k_{\lambda} \partial k_{\mu}}\right)_{0}\left(E_{0, i}^{*}(r) \nabla_{\mu} E_{0, j}(r)-E_{0, j}(r) \nabla_{\mu} E_{0, i}^{*}(r)\right)+\cdots\right\} .
\end{aligned}
$$

Thus we obtained a local conservation theorem of the form

$$
\nabla_{\lambda} T_{\kappa \lambda}(r)=0 \text {, }
$$


with a tensor function $T_{\kappa \lambda}(r)$ which explicitly written down up to terms with first-order derivatives of the slowly varying amplitudes is given by

$$
\begin{aligned}
\alpha T_{\kappa \lambda}(r)= & -\left(\frac{\partial\left(k_{\kappa} L_{i j}\right)}{\partial k_{\lambda}}\right)_{0} E_{0, i}^{*}(r) E_{0, j}(r) \\
& +\frac{\mathrm{i}}{2}\left(\frac{\partial^{2}\left(k_{\kappa} L_{i j}\right)}{\partial k_{\lambda} \partial k_{\mu}}\right)_{0}\left(E_{0, i}^{*}(r) \nabla_{\mu} E_{0, j}(r)-E_{0, j}(r) \nabla_{\mu} E_{0, i}^{*}(r)\right)+\cdots
\end{aligned}
$$

The four-dimensional covariance of $T_{\kappa \lambda}(r)$ with respect to index $\lambda$ is the same as in the action 4-vector $T_{\lambda}(r)$ and the covariance with respect to index $\kappa$ is evident from construction (7.1) with 4 -wave vector $k_{\kappa}$. That this is connected with homogeneity (or translation invariance) in space and time is easily seen since in case of absence of this symmetry it is impossible to have globally constant wave vectors and frequencies as used in the derivation. Thus we have the justification to call $T_{\kappa \lambda}(r)$ the energy-momentum tensor of homogeneous anisotropic dispersive media in the approximation of quasiplane and quasimonochromatic waves. In general, the tensor $T_{\kappa \lambda}(r)$ is non-symmetric

$$
T_{\kappa \lambda}(r) \neq T_{\lambda \kappa}(r)
$$

and is, in general, not equivalent to a symmetric one that means it is intrinsically non-symmetric.

We now transform the energy-momentum tensor $T_{\kappa \lambda}(r)$ to another form which is interesting for the physical interpretation. For this purpose we use the identities

$$
\begin{aligned}
& \left(\frac{\partial\left(k_{\kappa} L_{i j}\right)}{\partial k_{\lambda}}\right)_{0}=k_{0, \kappa}\left(\frac{\partial L_{i j}}{\partial k_{\lambda}}\right)_{0}+\delta_{\kappa \lambda}\left(L_{i j}\right)_{0}, \\
& \left(\frac{\partial^{2}\left(k_{\kappa} L_{i j}\right)}{\partial k_{\lambda} \partial k_{\mu}}\right)_{0}=k_{0, \kappa}\left(\frac{\partial^{2} L_{i j}}{\partial k_{\lambda} \partial k_{\mu}}\right)_{0}+\delta_{\kappa \mu}\left(\frac{\partial L_{i j}}{\partial k_{\lambda}}\right)_{0}+\delta_{\kappa \lambda}\left(\frac{\partial L_{i j}}{\partial k_{\mu}}\right)_{0} .
\end{aligned}
$$

Inserting this into (7.3) and using the representation (6.9) of $T_{\lambda}(r)$ and the Equations (6.5) and (6.6) for the slowly varying amplitudes we obtain up to first-order derivatives of these amplitudes

$$
\begin{aligned}
\alpha T_{\kappa \lambda}(r)= & \alpha k_{0, \kappa} T_{\lambda}(r)-\delta_{\kappa \lambda}\left(L_{i j}\right)_{0} E_{0, i}^{*}(r) E_{0, j}(r) \\
& +\frac{\mathrm{i}}{2}\left(\frac{\partial L_{i j}}{\partial k_{\lambda}}\right)_{0}\left(E_{0, i}^{*}(r) \nabla_{\kappa} E_{0, j}(r)-E_{0, j}(r) \nabla_{\kappa} E_{0, i}^{*}(r)\right)+\cdots .
\end{aligned}
$$

In the limiting transition from the slowly varying amplitudes to constant amplitudes the terms with derivatives of these amplitudes vanish and $\left(L_{i j}\right)_{0} E_{0, j}=0$ and we obtain the factorization

$$
T_{\kappa \lambda}(r)=k_{0, \kappa} T_{\lambda}(r),
$$

of the energy-momentum tensor. This is in full analogy to a homogeneous particle flow as discussed in e.g. [3] [12] (see also [34] and below) where, however, macroscopic electrodynamics provides a greater variety of possible dependencies 
of the momentum of one particle on the group velocity than classical mechanics. Taking seriously this analogy to a homogeneous particle flow this leads in a natural way to a quantization of the electrodynamic flow and to its interpretation as a flow of quasiparticles. The energy-momentum tensor $T_{\kappa \lambda}(r)$ in higher approximations according to (7.6) does not fully factorize into the product $k_{0, \kappa} T_{\lambda}(r)$ and the remaining terms are important at such space-time points where the 4-gradient of the slowly varying amplitudes of the electric field components is important. This may be interpreted as the tendency that energy and momentum flow at these points are forced to choose deviating directions in comparison to the homogeneous particle flow and expresses some interaction of the particles within the flow or some (direction-dependent) pressure or stress. This is in rough agreement with the diffraction of beams, for example, of Gaussian beams which cannot remain to be focused over the whole length of the beam.

\section{Three-Dimensional Representation of Energy-Momentum Tensor}

We now make the transition to the three-dimensional separation of the terms in the local laws of momentum and of energy conservation. The 4-dimensional energy-momentum tensor can be separated into three-dimensional parts in the following way defining (in common sense) the introduced new quantities on the right-hand side

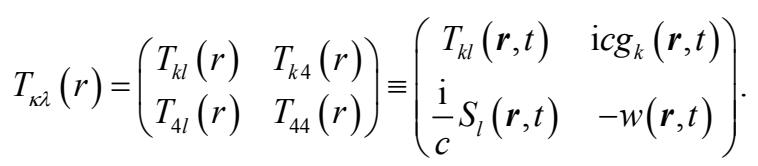

Then from (7.2) we find the following differential law of momentum conservation

$$
\nabla_{l} T_{k l}(\boldsymbol{r}, t)+\frac{1}{\mathrm{i} c} \frac{\partial}{\partial t} T_{k 4}(\boldsymbol{r}, t)=0, \Leftrightarrow \nabla_{l} T_{k l}(\boldsymbol{r}, t)+\frac{\partial}{\partial t} g_{k}(\boldsymbol{r}, t)=0,
$$

where $T_{k l}(\boldsymbol{r}, t)$ is the (Maxwell) stress tensor and $g_{k}(\boldsymbol{r}, t)$ the momentum density $^{8}$. Furthermore, the following differential law of energy conservation holds

$$
\nabla_{l} T_{4 l}(\boldsymbol{r}, t)+\frac{1}{\mathrm{i} c} \frac{\partial}{\partial t} T_{44}(\boldsymbol{r}, t)=0, \Leftrightarrow \nabla_{l} S_{l}(\boldsymbol{r}, t)+\frac{\partial}{\partial t} w(\boldsymbol{r}, t)=0,
$$

where $S_{l}(\boldsymbol{r}, t)$ is the energy flow density (Poynting vector) and $w(\boldsymbol{r}, t)$ the energy density.

According to (7.3) and (7.6) taking into account (8.1) the stress tensor possesses the form

\footnotetext{
${ }^{8}$ The three-dimensional stress tensor $T_{k l}$ is sometimes defined with opposite sign. Our sign of $T_{k l}$ agrees with that in the same notation $T_{k l}=T^{k l}$ in Landau and Lifshits [3] (Ed. 1962) and with $T_{k l} \equiv-\sigma_{k l}$ in later editions (e.g., [45] from 1988). Apparently, the notation $\sigma_{k l}$ agrees also with respect to sign to the same notation in [13] and in [10].
} 


$$
\begin{aligned}
\alpha T_{k l}(\boldsymbol{r}, t)= & -\left(\frac{\partial\left(k_{k} L_{i j}\right)}{\partial k_{l}}\right)_{0} E_{0, i}^{*}(\boldsymbol{r}, t) E_{0, j}(\boldsymbol{r}, t) \\
& +\frac{\mathrm{i}}{2}\left(\frac{\partial^{2}\left(k_{k} L_{i j}\right)}{\partial k_{l} \partial k_{m}}\right)_{0}\left(E_{0, i}^{*}(\boldsymbol{r}, t) \nabla_{m} E_{0, j}(\boldsymbol{r}, t)-E_{0, j}(\boldsymbol{r}, t) \nabla_{m} E_{0, i}^{*}(\boldsymbol{r}, t)\right) \\
& -\frac{\mathrm{i}}{2}\left(\frac{\partial^{2}\left(k_{k} L_{i j}\right)}{\partial k_{l} \partial \omega}\right)_{0}\left(E_{0, i}^{*}(\boldsymbol{r}, t) \frac{\partial}{\partial t} E_{0, j}(\boldsymbol{r}, t)-E_{0, j}(\boldsymbol{r}, t) \frac{\partial}{\partial t} E_{0, i}^{*}(\boldsymbol{r}, t)\right)+\cdots,
\end{aligned}
$$

and the momentum density is

$$
\begin{aligned}
\alpha g_{k}(\boldsymbol{r}, t)= & \left(\frac{\partial\left(k_{k} L_{i j}\right)}{\partial \omega}\right)_{0} E_{0, i}^{*}(\boldsymbol{r}, t) E_{0, j}(\boldsymbol{r}, t) \\
& -\frac{\mathrm{i}}{2}\left(\frac{\partial^{2}\left(k_{k} L_{i j}\right)}{\partial \omega \partial k_{m}}\right)_{0}\left(E_{0, i}^{*}(\boldsymbol{r}, t) \nabla_{m} E_{0, j}(\boldsymbol{r}, t)-E_{0, j}(\boldsymbol{r}, t) \nabla_{m} E_{0, i}^{*}(\boldsymbol{r}, t)\right) \\
& +\frac{\mathrm{i}}{2}\left(\frac{\partial^{2}\left(k_{k} L_{i j}\right)}{\partial \omega^{2}}\right)_{0}\left(E_{0, i}^{*}(\boldsymbol{r}, t) \frac{\partial}{\partial t} E_{0, j}(\boldsymbol{r}, t)-E_{0, j}(\boldsymbol{r}, t) \frac{\partial}{\partial t} E_{0, i}^{*}(\boldsymbol{r}, t)\right)+\cdots
\end{aligned}
$$

For the energy flow density we find from (7.3) and (7.6) taking into account (8.1)

$$
\begin{aligned}
\alpha S_{l}(\boldsymbol{r}, t)= & -\left(\frac{\partial\left(\omega L_{i j}\right)}{\partial k_{l}}\right)_{0} E_{0, i}^{*}(\boldsymbol{r}, t) E_{0, j}(\boldsymbol{r}, t) \\
& +\frac{\mathrm{i}}{2}\left(\frac{\partial^{2}\left(\omega L_{i j}\right)}{\partial k_{l} \partial k_{m}}\right)_{0}\left(E_{0, i}^{*}(\boldsymbol{r}, t) \nabla_{m} E_{0, j}(\boldsymbol{r}, t)-E_{0, j}(\boldsymbol{r}, t) \nabla_{m} E_{0, i}^{*}(\boldsymbol{r}, t)\right) \\
& -\frac{\mathrm{i}}{2}\left(\frac{\partial^{2}\left(\omega L_{i j}\right)}{\partial k_{l} \partial \omega}\right)_{0}\left(E_{0, i}^{*}(\boldsymbol{r}, t) \frac{\partial}{\partial t} E_{0, j}(\boldsymbol{r}, t)-E_{0, j}(\boldsymbol{r}, t) \frac{\partial}{\partial t} E_{0, i}^{*}(\boldsymbol{r}, t)\right)+\cdots,
\end{aligned}
$$

and for the energy density

$$
\begin{aligned}
\alpha w(\boldsymbol{r}, t)= & \left.\frac{\partial\left(\omega L_{i j}\right)}{\partial \omega}\right)_{0} E_{0, i}^{*}(\boldsymbol{r}, t) E_{0, j}(\boldsymbol{r}, t) \\
& -\frac{\mathrm{i}}{2}\left(\frac{\partial^{2}\left(\omega L_{i j}\right)}{\partial \omega \partial k_{m}}\right)_{0}\left(E_{0, i}^{*}(\boldsymbol{r}, t) \nabla_{m} E_{0, j}(\boldsymbol{r}, t)-E_{0, j}(\boldsymbol{r}, t) \nabla_{m} E_{0, i}^{*}(\boldsymbol{r}, t)\right) \\
& +\frac{\mathrm{i}}{2}\left(\frac{\partial^{2}\left(\omega L_{i j}\right)}{\partial \omega^{2}}\right)_{0}\left(E_{0, i}^{*}(\boldsymbol{r}, t) \frac{\partial}{\partial t} E_{0, j}(\boldsymbol{r}, t)-E_{0, j}(\boldsymbol{r}, t) \frac{\partial}{\partial t} E_{0, i}^{*}(\boldsymbol{r}, t)\right)+\cdots .
\end{aligned}
$$

The terms with spatial and temporal derivatives of the slowly varying amplitudes describe in addition to the stable form of propagation of a wave group its diffraction.

Integral forms of the conservation of momentum and energy in time follow from integration of the conservation theorems within a volume $V$ with surface $S$ and normal unit-vector $N$ directed to the inside of the surface $S$ by (Gauss 
theorem)

$\frac{\partial}{\partial t} P_{k}(t) \equiv \frac{\partial}{\partial t} \int_{V} \mathrm{~d} V(\boldsymbol{r}) g_{k}(\boldsymbol{r}, t)=-\int_{V} \mathrm{~d} V(\boldsymbol{r}) \nabla_{l} T_{k l}(\boldsymbol{r}, t)=+\oint_{S} \mathrm{~d} S(\boldsymbol{r}) T_{k l}(\boldsymbol{r}, t) N_{l}(\boldsymbol{r})$,

$\frac{\partial}{\partial t} E(t) \equiv \frac{\partial}{\partial t} \int_{V} \mathrm{~d} V(\boldsymbol{r}) w(\boldsymbol{r}, t)=-\int_{V} \mathrm{~d} V(\boldsymbol{r}) \nabla_{l} S_{l}(\boldsymbol{r}, t)=+\oint_{S} \mathrm{~d} S(\boldsymbol{r}) S_{l}(\boldsymbol{r}, t) N_{l}(\boldsymbol{r})$,

with $P_{\kappa} \equiv\left(P_{k}, P_{4}=\mathrm{i} \frac{E}{c}\right)$ the four-vector of momentum 9 .

\section{Limiting Transition to Plane Monochromatic Waves in Anisotropic Dispersive Media}

In the limiting transition from quasiplane and quasimonochromatic waves to plane monochromatic waves the slowly varying amplitudes become constant amplitudes $\boldsymbol{E}_{0}(\boldsymbol{r}, t) \rightarrow \boldsymbol{E}_{0}$ and the energy-momentum tensor becomes independent on the space-time points $(\boldsymbol{r}, t)$ that means $T_{\kappa \lambda}(\boldsymbol{r}, t) \rightarrow T_{\kappa \lambda}$. The propagation of the wave as a wave packet with the group velocity in this limiting transition becomes the more invisible the nearer it comes to a plane monochromatic wave.

From (7.3) together with (7.5) or from (7.6) follows in this limiting transition in relativistic covariant form

$$
\alpha T_{\kappa \lambda}=-E_{0, i}^{*}\left(\frac{\partial\left(k_{\kappa} L_{i j}\right)}{\partial k_{\lambda}}\right)_{0} E_{0, j}=-k_{0, \kappa} E_{0, i}^{*}\left(\frac{\partial L_{i j}}{\partial k_{\lambda}}\right)_{0} E_{0, j}=k_{0, \kappa} \alpha T_{\lambda} .
$$

In the three-dimensional separation expressed by the formulae (8.4), (8.5), (8.6) and (8.7) this limiting transition results in the relations

$$
\alpha T_{\kappa \lambda}=\left(\begin{array}{cc}
-E_{0, i}^{*}\left(\frac{\partial\left(k_{k} L_{i j}\right)}{\partial k_{l}}\right)_{0} E_{0, j} & \mathrm{i} c E_{0, i}^{*}\left(\frac{\partial\left(k_{k} L_{i j}\right)}{\partial \omega}\right)_{0} E_{0, j} \\
-\frac{\mathrm{i}}{c} E_{0, i}^{*}\left(\frac{\partial\left(\omega L_{i j}\right)}{\partial k_{l}}\right)_{0} E_{0, j} & -E_{0, i}^{*}\left(\frac{\partial\left(\omega L_{i j}\right)}{\partial \omega}\right)_{0} E_{0, j}
\end{array}\right)=\alpha\left(\begin{array}{cc}
k_{0, k} T_{l} & \mathrm{i} c k_{0, k} s \\
\frac{\mathrm{i}}{c} \omega_{0} T_{l} & -\omega_{0} s
\end{array}\right),
$$

where the action flow density $T_{l}$ and the action density $s$ according to (6.12) become

$$
\alpha T_{l}=-E_{0, i}^{*}\left(\frac{\partial L_{i j}}{\partial k_{l}}\right)_{0} E_{0, j}, \quad \alpha s=E_{0, i}^{*}\left(\frac{\partial L_{i j}}{\partial \omega}\right)_{0} E_{0, j}
$$

${ }^{9}$ The sign of $N_{l}(\boldsymbol{r})$ at the surface element $\mathrm{d} S$ can be verified from the derivative of the characteristic function $\theta_{V}(\boldsymbol{r}) \equiv\left\{\begin{array}{l}1, \boldsymbol{r} \in V \\ 0, \boldsymbol{r} \notin V\end{array}\right.$ of the volume $V$ which is $\nabla_{l} \theta_{V}(\boldsymbol{r})=N_{l}(\boldsymbol{r}) \delta_{S}(\boldsymbol{r})$ with normal unit vector $N_{l}(\boldsymbol{r})$ directed to the inside of $\mathrm{d} S(\boldsymbol{r})$ as generalization of the step function and of the delta function as its derivative according to

$$
\begin{aligned}
\int_{V} \mathrm{~d} V(\boldsymbol{r})\left(\nabla_{l} A_{l}(\boldsymbol{r})\right) & =\int \mathrm{d} V(\boldsymbol{r}) \theta_{V}(\boldsymbol{r})\left(\nabla_{l} A_{l}(\boldsymbol{r})\right) \\
& =\underbrace{\int \mathrm{d} V(\boldsymbol{r}) \nabla_{l}\left(\theta_{V}(\boldsymbol{r}) A_{l}(\boldsymbol{r})\right)}_{=0}-\int \mathrm{d} V(\boldsymbol{r}) A_{l}(\boldsymbol{r})\left(\nabla_{l} \theta_{V}(\boldsymbol{r})\right) \\
& =-\oint_{S} \mathrm{~d} S(\boldsymbol{r}) A_{l}(\boldsymbol{r}) N_{l}(\boldsymbol{r}),
\end{aligned}
$$

where integrals without given boundaries go over the whole space. 
We will show in the following that these expressions are not in contradiction to known expressions for the energy-momentum tensor (mostly more special or otherwise formulated ones).

If we use the explicit form of $L_{i j}(\boldsymbol{k}, \omega)$ given in (5.5) we obtain from (9.2)

$$
\begin{aligned}
\alpha T_{k l}= & \frac{c^{2}}{\omega_{0}^{2}} E_{0, i}^{*}\left\{\left(\boldsymbol{k}_{0}^{2} \delta_{i j}-k_{0, i} k_{0, j}\right) \delta_{k l}+k_{0, k}\left(2 k_{0, l} \delta_{i j}-k_{0, i} \delta_{j l}-k_{0, j} \delta_{i l}\right)\right\} E_{0, j} \\
& -E_{0, i}^{*}\left(\frac{\partial\left(k_{k} \varepsilon_{i j}\right)}{\partial k_{l}}\right)_{0} E_{0, j}, \\
\alpha g_{k}= & 2 \frac{c^{2}}{\omega_{0}^{3}} k_{0, k} E_{0, i}^{*}\left(\boldsymbol{k}_{0}^{2} \delta_{i j}-k_{0, i} k_{0, j}\right) E_{0, j}+k_{0, k} E_{0, i}^{*}\left(\frac{\partial \varepsilon_{i j}}{\partial \omega}\right)_{0} E_{0, j}, \\
\alpha S_{l}= & \frac{c^{2}}{\omega_{0}} E_{0, i}^{*}\left(2 k_{0, l} \delta_{i j}-k_{0, i} \delta_{j l}-k_{0, j} \delta_{i l}\right) E_{0, j}-\omega_{0} E_{0, i}^{*}\left(\frac{\partial \varepsilon_{i j}}{\partial k_{l}}\right)_{0} E_{0, j}, \\
\alpha w= & \frac{c^{2}}{\omega_{0}^{2}} E_{0, i}^{*}\left(\boldsymbol{k}_{0}^{2} \delta_{i j}-k_{0, i} k_{0, j}\right) E_{0, j}+E_{0, i}^{*}\left(\frac{\partial\left(\omega \varepsilon_{i j}\right)}{\partial \omega}\right)_{0} E_{0, j},
\end{aligned}
$$

and from (9.3) for action flow density $T_{l}$ and action density $s$

$$
\begin{aligned}
& \alpha T_{l}=\frac{c^{2}}{\omega_{0}^{2}} E_{0, i}^{*}\left(2 k_{0, l} \delta_{i j}-k_{0, i} \delta_{j l}-k_{0, j} \delta_{i l}\right) E_{0, j}-E_{0, i}^{*}\left(\frac{\partial \varepsilon_{i j}}{\partial k_{l}}\right)_{0} E_{0, j}, \\
& \alpha s=2 \frac{c^{2}}{\omega_{0}^{3}} E_{0, i}^{*}\left(\boldsymbol{k}_{0}^{2} \delta_{i j}-k_{0, i} k_{0, j}\right) E_{0, j}+E_{0, i}^{*}\left(\frac{\partial \varepsilon_{i j}}{\partial \omega}\right)_{0} E_{0, j} .
\end{aligned}
$$

As already discussed, in the transition to the factorized form in (9.1) and (9.2) we used the equations for the slowly varying amplitudes which after transition to plane monochromatic waves become the algebraic equations $\left(L_{i j}\right)_{0} E_{0, j}=0$ and $E_{0, i}^{*}\left(L_{i j}\right)_{0}=0$. Instead of these equations we can also directly use the equations of macroscopic electrodynamics (2.7) which for plane monochromatic waves with real wave vector $\boldsymbol{k}_{0}$ and real frequency $\omega_{0}$ take on the form ${ }^{10}$

$$
\begin{aligned}
& \boldsymbol{B}_{0}=\frac{c}{\omega_{0}}\left[\boldsymbol{k}_{0}, \boldsymbol{E}_{0}\right], \quad \boldsymbol{D}_{0}=-\frac{c}{\omega_{0}}\left[\boldsymbol{k}_{0}, \boldsymbol{B}_{0}\right], \Rightarrow \boldsymbol{k}_{0} \boldsymbol{B}_{0}=\boldsymbol{k}_{0} \boldsymbol{D}_{0}=0, \\
& \boldsymbol{B}_{0}^{*}=\frac{c}{\omega_{0}}\left[\boldsymbol{k}_{0}, \boldsymbol{E}_{0}^{*}\right], \quad \boldsymbol{D}_{0}^{*}=-\frac{c}{\omega_{0}}\left[\boldsymbol{k}_{0}, \boldsymbol{B}_{0}^{*}\right], \Rightarrow \boldsymbol{k}_{0} \boldsymbol{B}_{0}^{*}=\boldsymbol{k}_{0} \boldsymbol{D}_{0}^{*}=0 .
\end{aligned}
$$

Using these equations, we can transform (9.4) exactly to the following "mixed" forms of representation with the amplitudes of the electric and magnetic field $\boldsymbol{E}_{0}$ and $\boldsymbol{B}_{0}$ and the electric induction $\boldsymbol{D}_{0}$ which dominate in their kind in literature (compare also [7] [9] [10]).

$$
\begin{aligned}
\alpha T_{k l}= & \left(\boldsymbol{B}_{0}^{*} \boldsymbol{B}_{0}+\frac{1}{2}\left(\boldsymbol{E}_{0}^{*} \boldsymbol{D}_{0}+\boldsymbol{D}_{0}^{*} \boldsymbol{E}_{0}\right)\right) \delta_{k l}-\left(B_{0, k}^{*} B_{0, l}+B_{0, k} B_{0, l}^{*}+E_{0, k}^{*} D_{0, l}+E_{0, k} D_{0, l}^{*}\right) \\
& -k_{0, k} \boldsymbol{E}_{0}^{*}\left(\frac{\partial \boldsymbol{\varepsilon}}{\partial k_{l}}\right)_{0} \boldsymbol{E}_{0}=\alpha k_{0, k} T_{l},
\end{aligned}
$$

${ }^{10} \mathrm{We}$ emphasize again that this restriction to real wave vectors and frequencies is not a principal restriction for lossless media but simplifies our derivations considerably since it does not introduce additional difficulties with inhomogeneous (evanescent) waves in lossless media which necessarily are to be discussed without this restriction. 


$$
\begin{aligned}
& \alpha g_{k}=\frac{1}{c}\left(\left[\boldsymbol{D}_{0}^{*}, \boldsymbol{B}_{0}\right]_{k}+\left[\boldsymbol{D}_{0}, \boldsymbol{B}_{0}^{*}\right]_{k}\right)+k_{0, k} \boldsymbol{E}_{0}^{*}\left(\frac{\partial \boldsymbol{\varepsilon}}{\partial \omega}\right)_{0} \boldsymbol{E}_{0}=\alpha k_{0, k} s, \\
& \alpha S_{l}=c\left(\left[\boldsymbol{E}_{0}^{*}, \boldsymbol{B}_{0}\right]_{l}+\left[\boldsymbol{E}_{0}, \boldsymbol{B}_{0}^{*}\right]_{l}\right)-\omega_{0} \boldsymbol{E}_{0}^{*}\left(\frac{\partial \boldsymbol{\varepsilon}}{\partial k_{l}}\right)_{0} \boldsymbol{E}_{0}=\alpha \omega_{0} T_{l}, \\
& \alpha w=\boldsymbol{B}_{0}^{*} \boldsymbol{B}_{0}+\frac{1}{2}\left(\boldsymbol{E}_{0}^{*} \boldsymbol{D}_{0}+\boldsymbol{D}_{0}^{*} \boldsymbol{E}_{0}\right)+\omega_{0} \boldsymbol{E}_{0}^{*}\left(\frac{\partial \boldsymbol{\varepsilon}}{\partial \omega}\right)_{0} \boldsymbol{E}_{0}=\alpha \omega_{0} s .
\end{aligned}
$$

For the action flow density $T_{l}$ and the action density $s$, we find

$$
\begin{aligned}
\alpha T_{l} & =\frac{c}{\omega_{0}}\left(\left[\boldsymbol{E}_{0}^{*}, \boldsymbol{B}_{0}\right]_{l}+\left[\boldsymbol{E}_{0}, \boldsymbol{B}_{0}^{*}\right]_{l}\right)-E_{0}^{*}\left(\frac{\partial \boldsymbol{\varepsilon}}{\partial k_{l}}\right)_{0} \boldsymbol{E}_{0}, \\
\alpha s & =\frac{1}{\omega_{0}}\left(\boldsymbol{B}_{0}^{*} \boldsymbol{B}_{0}+\frac{1}{2}\left(\boldsymbol{E}_{0}^{*} \boldsymbol{D}_{0}+\boldsymbol{D}_{0}^{*} \boldsymbol{E}_{0}\right)\right)+\boldsymbol{E}_{0}^{*}\left(\frac{\partial \boldsymbol{\varepsilon}}{\partial \omega}\right)_{0} \boldsymbol{E}_{0} \\
& =\frac{c}{\omega_{0}^{2}} \boldsymbol{k}_{0}\left(\left[\boldsymbol{E}_{0}^{*}, \boldsymbol{B}_{0}\right]+\left[\boldsymbol{E}_{0}, \boldsymbol{B}_{0}^{*}\right]\right)+\boldsymbol{E}_{0}^{*}\left(\frac{\partial \boldsymbol{\varepsilon}}{\partial \omega}\right)_{0} \boldsymbol{E}_{0} .
\end{aligned}
$$

The appearance of $\omega_{0}$ in the denominators for action flow density $T_{l}$ and action density $s$ shows that they are formed in nonlocal way by the fields that in the space-time picture is impossible to express by quadratic local field combinations only and which, perhaps, is a reason that they did not find much attention (exception: similar considerations to adiabatic invariance).

We see that all parts of the energy-momentum tensor in (9.7) contain a part with origin from the dispersion of the medium. The momentum density $g_{k}$ which possesses the direction of the mean wave vector $k_{0, k}$ and the energy density $w$ are modified by terms $\left(\frac{\partial \varepsilon_{i j}}{\partial \omega}\right)_{0}$ with derivatives of the permittivity tensor $\varepsilon_{i j}(\boldsymbol{k}, \omega)$ with respect to frequency $\omega$ (this goes back to Brillouin in 1921; see also [7] [9] but many other, in principle, excellent monographs on electrodynamics and optics do not take this into account). The stress tensor $T_{k l}$ and the energy flow density $S_{l}$ are modified by terms $\left(\frac{\partial \varepsilon_{i j}}{\partial k_{l}}\right)_{0}$ with derivatives of the permittivity tensor with respect to the wave vector $\boldsymbol{k}$ which are non-vanishing only in case of presence of spatial dispersion (see, e.g., [9] [10] [11]). The terms from frequency dispersion may become very important in the neighborhood of eigenfrequencies of the medium (e.g., such as used for laser transitions) and do not represent in this case only a small correction to the terms without dispersion. The terms with spatial dispersion are non-vanishing, for example, for media with natural optical activity or for hot gases and plasmas. If a medium possesses only frequency dispersion $\varepsilon_{i j}(\boldsymbol{k}, \omega) \equiv \varepsilon_{i j}(\omega)$ in inertial system $\mathcal{I}$ then by transition to an inertial system $\mathcal{I}^{\prime}$ where this medium is moving the new permittivity tensor $\varepsilon_{i j}^{\prime}\left(\boldsymbol{k}^{\prime}, \omega^{\prime}\right)$ in $\mathcal{I}^{\prime}$ depends apart from transformed frequency $\omega^{\prime}$ also on transformed wave vector $\boldsymbol{k}^{\prime}$ and appears there as medium with "unnatural" spatial dispersion (see Appendix A).

The trace of the energy-momentum tensor which is a relativistic invariant is non-vanishing taking into account the dispersion. From the limiting case of 
plane monochromatic waves in (9.7) we find

$$
\alpha(\langle\mathrm{T}\rangle-w)=-k_{0, k} E_{0, i}^{*}\left(\frac{\partial \varepsilon_{i j}}{\partial k_{k}}\right)_{0} E_{0, j}-\omega_{0} E_{0, i}^{*}\left(\frac{\partial \varepsilon_{i j}}{\partial \omega}\right)_{0} E_{0, j} .
$$

If we neglect dispersion the trace of the energy-momentum tensor becomes vanishing as it is seen from this expression. Due to factorization of the stress tensor $T_{k l}=k_{0, k} T_{l}$ and of the whole energy-momentum tensor $T_{\kappa \lambda}$ they possess only one non-vanishing eigenvalue and the quantity $[T] \equiv \frac{1}{2}\left(\langle\mathrm{~T}\rangle^{2}-\left\langle\mathrm{T}^{2}\right\rangle\right)$ involved in the second invariant $[T] \equiv[\mathrm{T}]-\langle\mathrm{T}\rangle w+\boldsymbol{S g}$ of the four-dimensional tensor $T$ (see (B.8) in Appendix B) is vanishing. Therefore, using the form (9.7) in considered approximation one can check the vanishing of the second invariant of the energy-momentum tensor

$$
[T]=\underbrace{[\mathrm{T}]}_{=0}-(\langle\mathrm{T}\rangle w-\boldsymbol{S g})=0
$$

which is a Lorentz-invariant and thus this relation is true in arbitrary inertial systems. Due to factorization (9.1) of the energy-momentum tensor in considered approximation we find

$$
\boldsymbol{S g}-\langle\mathrm{T}\rangle w=0
$$

remaining true after Lorentz transformation.

The energy-momentum tensor (9.7) is intrinsically non-symmetric expressed by relation (7.4) also under neglect of dispersion. In general, for anisotropic media the momentum density $g_{k}$ and the energy flow density $S_{l}$ possess different directions and there is no way to remove this but also the stress tensor $T_{k l}$ is non-symmetric for anisotropic media. From the two old proposals for this tensor which are the Minkowski tensor and the Abraham tensor (see, e.g., [5] [10]) the tensor (9.2) is nearer to the Minkowski tensor and makes the transition to it in case of neglected dispersion. However, this problem of the correct tensor did not genuinely exist in our derivations since under the condition (3.9) that the medium is lossless the local form of the conservation laws could be formulated as exact vanishing of a 4-divergence of an energy-momentum tensor.

We can subdivide the energy-momentum tensor $T_{\kappa \lambda}$ in (9.7) in additive way into a pure electromagnetic field tensor $T_{\kappa \lambda}^{(\mathrm{F})}$ which contains only the electric field $\boldsymbol{E}$ and magnetic field $\boldsymbol{B}$ and a field-matter interaction tensor $T_{\kappa \lambda}^{(\mathrm{I})}$ which contains in addition the polarization $\boldsymbol{P}=\frac{1}{4 \pi}(\boldsymbol{D}-\boldsymbol{E})$ and derivatives of the permittivity tensor $\varepsilon_{i j}(\boldsymbol{k}, \omega)$. The field part which is quadratic in the electromagnetic field is then a symmetric tensor $T_{\kappa \lambda}^{(\mathrm{F})}=T_{\lambda \kappa}^{(\mathrm{F})}$ and equal in form to the tensor for vacuum. The interaction part $T_{\kappa \lambda}^{(\mathrm{I})}$ which is bilinear in field and polarization or contains derivatives of the permittivity tensor is non-symmetric. Their explicit forms may be taken from (9.7). It should be emphasized that such a subdivision remains to be formally since each of the two parts does not separately obey a local conservation law. 
In the transition to vacuum $\varepsilon_{i j}(\boldsymbol{k}, \omega) \rightarrow \delta_{i j}$ from (9.7) we obtain a symmetric energy-momentum tensor and it possesses at the same time the most simple form in comparison to equivalent ones since it does not contain parts with the rapidly varying phase factors $\mathrm{e}^{ \pm \mathrm{i} 2\left(\boldsymbol{k}_{0} \boldsymbol{r}-\omega_{0} t\right)}$ and admits a direct physical interpretation by considerations about the group velocity.

\section{Group Velocity in Energy-Momentum Tensor for Anisotropic Dispersive Media and Its Calculation}

A wave packet in a homogeneous medium propagates in first approximation with shape stability and without diffraction with the group velocity and therefore energy and momentum of this wave packet should propagate also with the group velocity. The introduction of the group velocity into the energy-momentum tensor in the limiting case of plane monochromatic wave reveals a simple basic structure of this tensor (see also, [7] [9] [10]).

Plane monochromatic waves with real wave vector and real frequency satisfy Equation (5.4) and together with the condition (5.13) for absent losses this can be written in operator form (i.e. without vectorial indices $L_{i j}(\boldsymbol{k}, \omega) \rightarrow \mathrm{L}(\boldsymbol{k}, \omega)$ ) as the following eigenvalue equations for right-hand and left-hand eigenvectors of $\mathrm{L}(\boldsymbol{k}, \omega)$ to eigenvalue zero

$$
\mathrm{L}(\boldsymbol{k}, \omega) \boldsymbol{E}(\boldsymbol{k}, \omega)=\mathbf{0}, \quad \boldsymbol{E}^{*}(\boldsymbol{k}, \omega) \mathrm{L}(\boldsymbol{k}, \omega)=\mathbf{0},
$$

with the operator $\mathrm{L}(\boldsymbol{k}, \omega)$ defined by (5.5) ( $\boldsymbol{a} \cdot \boldsymbol{b}$ is dyadic product of vectors $\boldsymbol{a}$ and $\boldsymbol{b}$ )

$$
\mathrm{L}(\boldsymbol{k}, \omega) \equiv \frac{c^{2}}{\omega^{2}}\left(\boldsymbol{k} \cdot \boldsymbol{k}-\boldsymbol{k}^{2} \mathrm{I}\right)+\boldsymbol{\varepsilon}(\boldsymbol{k}, \omega) .
$$

The necessary condition for solutions of the operator Equations (10.1) is the vanishing of the determinant $|\mathrm{L}(\boldsymbol{k}, \omega)|$ of the operator $\mathrm{L}(\boldsymbol{k}, \omega)$

$$
|\mathrm{L}(\boldsymbol{k}, \omega)|=0 \text {, }
$$

which in coordinate-invariant notation is explicitly given by [27] [28] [31] ${ }^{11}$

$$
\begin{aligned}
|\mathrm{L}(\boldsymbol{k}, \omega)| & =\frac{c^{4}}{\omega^{4}} \boldsymbol{k}^{2}(\boldsymbol{k} \boldsymbol{\varepsilon} \boldsymbol{k})-\frac{c^{2}}{\omega^{2}}\left(\langle\boldsymbol{\varepsilon}\rangle \boldsymbol{k} \boldsymbol{\varepsilon} \boldsymbol{k}-\boldsymbol{k} \boldsymbol{\varepsilon}^{2} \boldsymbol{k}\right)+|\boldsymbol{\varepsilon}| \\
& =\frac{c^{4}}{\omega^{4}} \boldsymbol{k}^{2}(\boldsymbol{k} \boldsymbol{\varepsilon} \boldsymbol{k})-\frac{c^{2}}{\omega^{2}}\left(\langle\overline{\boldsymbol{\varepsilon}}\rangle \boldsymbol{k}^{2}-\boldsymbol{k} \overline{\boldsymbol{\varepsilon}} \boldsymbol{k}\right)+|\boldsymbol{\varepsilon}|, \quad \boldsymbol{\varepsilon} \equiv \boldsymbol{\varepsilon}(\boldsymbol{k}, \omega),
\end{aligned}
$$

where $\langle\varepsilon\rangle$ denotes the trace and $|\varepsilon|$ the determinant of the permittivity tensor and where $\boldsymbol{k} \boldsymbol{\varepsilon} \boldsymbol{k} \equiv k_{i} \varepsilon_{i j} k_{j}$ and $\boldsymbol{k} \boldsymbol{\varepsilon}^{2} \boldsymbol{k} \equiv k_{i} \varepsilon_{i j} \varepsilon_{j k} k_{k}$ in notation with three-dimensional vector indices. The vanishing of the determinant (10.4) de-

\footnotetext{
${ }^{11}$ Fyodorov [27], Eq. (17.21) expresses the second sum term in round brackets by the inverse permittivity tensor $\varepsilon^{-1}$ or, more precisely, by the related complementary tensor

$\bar{\varepsilon} \equiv|\varepsilon| \varepsilon^{-1}=\varepsilon^{2}-\langle\varepsilon\rangle \varepsilon+[\varepsilon]$ I to $\varepsilon$ with the identity $\langle\bar{\varepsilon}\rangle=[\varepsilon] \equiv \frac{1}{2}\left(\langle\varepsilon\rangle^{2}-\left\langle\varepsilon^{2}\right\rangle\right)$ as here additionally given. Other (historically older) formulations of vanishing of this determinant in the form of the Fresnel equation in coordinates of the principal axes of the permittivity tensor are known (e.g., Szivessy [46] (Eqs. (47) and (48)) or Born and Wolf [47] (chap. XV.2.2, Eq. (21)) which correspond to (3) and (4) in coordinates of the principal axes and the same is true for Eq. (97.10) in [7]).
} 
scribes a three-dimensional (hyper-)surface in the four-dimensional space of variables $(\boldsymbol{k}, \omega)$ which is called dispersion surface.

The dispersion Equation (10.3) can be resolved with respect to one of the 4 components of $(\boldsymbol{k}, \omega)$, for example, in the form of the frequency $\omega$ as function of the wave vector $\boldsymbol{k}$ that means

$$
\omega=\omega(\boldsymbol{k}) .
$$

The group velocity $\boldsymbol{v}$ is then defined by

$$
\boldsymbol{v} \equiv \frac{\partial \omega}{\partial \boldsymbol{k}}(\boldsymbol{k})=\boldsymbol{v}(\boldsymbol{k})
$$

It is a "regular" velocity also in the relativistic theorem of addition of velocities. Inserting $\omega=\omega(\boldsymbol{k})$ in the dispersion Equation (10.3) we get a scalar identity as a function of the wave vector $\boldsymbol{k}$ from which after differentiation with respect to the wave vector follows a vector identity

$$
|\mathrm{L}(\boldsymbol{k}, \omega(\boldsymbol{k}))|=0, \Rightarrow\left(\frac{\partial|\mathrm{L}|}{\partial \boldsymbol{k}}\right)_{(\boldsymbol{k}, \omega(\boldsymbol{k}))}+\left(\frac{\partial|\mathrm{L}|}{\partial \omega}\right)_{(\boldsymbol{k}, \omega(\boldsymbol{k}))} \frac{\partial \omega}{\partial \boldsymbol{k}}(\boldsymbol{k})=\mathbf{0},
$$

with arguments of involved functions of $(\boldsymbol{k}, \omega)$ taken at $(\boldsymbol{k}, \omega(\boldsymbol{k}))$ and we obtain for the group velocity (vectorial indices of $\boldsymbol{v}$ and of $\frac{\partial}{\partial \boldsymbol{k}}$ correspond to each other on left- and right-hand sides)

$$
\boldsymbol{v}(k)=-\frac{\left(\frac{\partial|\mathrm{L}|}{\partial \boldsymbol{k}}\right)_{(\boldsymbol{k}, \omega(\boldsymbol{k}))}}{\left(\frac{\partial|\mathrm{L}|}{\partial \omega}\right)_{(\boldsymbol{k}, \omega(\boldsymbol{k}))}}=-\frac{\left\langle\overline{\mathrm{L}} \frac{\partial \mathrm{L}}{\partial \boldsymbol{k}}\right\rangle_{(\boldsymbol{k}, \omega(\boldsymbol{k}))}}{\left\langle\overline{\mathrm{L}} \frac{\partial \mathrm{L}}{\partial \omega}\right\rangle_{(\boldsymbol{k}, \omega(\boldsymbol{k}))}}=-\frac{\left(\boldsymbol{E}^{*} \frac{\partial \mathrm{L}}{\partial \boldsymbol{k}} \boldsymbol{E}\right)_{(\boldsymbol{k}, \omega(\boldsymbol{k}))}}{\left(\boldsymbol{E}^{*} \frac{\partial \mathrm{L}}{\partial \omega} \boldsymbol{E}\right)_{(\boldsymbol{k}, \omega(\boldsymbol{k}))}} .
$$

We applied here the relation $\frac{\partial|\mathrm{A}|}{\partial \lambda}=\left\langle\overline{\mathrm{A}} \frac{\partial \mathrm{A}}{\partial \lambda}\right\rangle$ for the differentiation of the determinant $|\mathrm{A}|$ of an arbitrary operator $\mathrm{A}$ with respect to a variable $\lambda$ where $\overline{\mathrm{A}}$ denotes the complementary operator to the operator $\mathrm{A}$ which satisfies the relations $\mathrm{A} \overline{\mathrm{A}}=\overline{\mathrm{A}} \mathrm{A}=|\mathrm{A}| \mathrm{I}$. The complementary operator $\overline{\mathrm{A}}$ to $\mathrm{A}$ is determined in components by $\bar{A}_{l i}=\frac{1}{2 !} \varepsilon_{i j k} \varepsilon_{l m n} A_{j m} A_{k n}$ or due to Hamilton-Cayley identity $A^{3}-\langle A\rangle A^{2}+[A] A-|A| I=0$ in operator form by $\overline{\mathrm{A}} \equiv \mathrm{A}^{2}-\langle\mathrm{A}\rangle \mathrm{A}+[\mathrm{A}] \mathrm{I}$ where $[\mathrm{A}] \equiv \frac{1}{2}\left(\langle\mathrm{~A}\rangle^{2}-\left\langle\mathrm{A}^{2}\right\rangle\right)=\langle\overline{\mathrm{A}}\rangle$ denotes the second invariant of $\mathrm{A}$ and $\mathrm{I}$ is the identity operator.

The explicit form of the complementary operator $\overline{\mathrm{L}}(\boldsymbol{k}, \omega)$ to $\mathrm{L}(\boldsymbol{k}, \omega)$ which by its vanishing determines the optic axes is (with $(\varepsilon \boldsymbol{k})_{i} \equiv \varepsilon_{i k} k_{k}$ and $(\boldsymbol{k} \varepsilon)_{j} \equiv k_{l} \varepsilon_{l j}$ in coordinates)

$$
\overline{\mathrm{L}}(\boldsymbol{k}, \omega)=\frac{c^{4}}{\omega^{4}}\left(\boldsymbol{k}^{2}\right) \boldsymbol{k} \cdot \boldsymbol{k}-\frac{c^{2}}{\omega^{2}}(\langle\boldsymbol{\varepsilon}\rangle \boldsymbol{k} \cdot \boldsymbol{k}-\boldsymbol{\varepsilon} \boldsymbol{k} \cdot \boldsymbol{k}-\boldsymbol{k} \cdot \boldsymbol{k} \boldsymbol{\varepsilon}+(\boldsymbol{k} \boldsymbol{\varepsilon} \boldsymbol{k}) \mathrm{I})+\overline{\boldsymbol{\varepsilon}},
$$

with trace equal to $(\langle\overline{\mathrm{A}}\rangle=[\mathrm{A}]$ is a three-dimensional operators identity for ar- 
bitrary A )

$$
\langle\overline{\mathrm{L}}(\boldsymbol{k}, \omega)\rangle=[\mathrm{L}(\boldsymbol{k}, \omega)]=\frac{c^{4}}{\omega^{4}}\left(\boldsymbol{k}^{2}\right)^{2}-\frac{c^{2}}{\omega^{2}}\left(\langle\boldsymbol{\varepsilon}\rangle \boldsymbol{k}^{2}+\boldsymbol{k} \boldsymbol{\varepsilon} \boldsymbol{k}\right)+[\boldsymbol{\varepsilon}] .
$$

From the Hamilton-Cayley identity in the form $\mathrm{L} \overline{\mathrm{L}}=\overline{\mathrm{L}} \mathrm{L}=|\mathrm{L}| \mathrm{I}$ it becomes clear that on the dispersion surface $|\mathrm{L}|=0$ each non-vanishing vector $\overline{\mathrm{L}} \boldsymbol{a} \neq \mathbf{0}$ with appropriately chosen vector $\boldsymbol{a}$ is right-hand eigenvector of $\mathrm{L}$ to eigenvalue 0 and thus a solution $\boldsymbol{E}$ of Equation (10.1) and each non-vanishing vector $\boldsymbol{b} \overline{\mathrm{L}} \neq \mathbf{0}$ is left-hand eigenvector of $\mathrm{L}$ to eigenvalue 0 and thus a solution $\boldsymbol{E}^{*}$ of second Equation (10.1). Therefore, the normalized operator $\overline{\mathrm{L}}$ in case of $|\mathrm{L}|=0$ as follows by applying the Hamilton-Cayley identity is the normalized dyadic product $\boldsymbol{e} \cdot \boldsymbol{e}^{*} \propto \boldsymbol{E} \cdot \boldsymbol{E}^{*}$ and

$$
(|\mathrm{L}|=0), \quad \Pi \equiv \frac{\overline{\mathrm{L}}}{\langle\overline{\mathrm{L}}\rangle}=\frac{\overline{\mathrm{L}}}{[\mathrm{L}]}=\boldsymbol{e} \cdot \boldsymbol{e}^{*}, \quad \Pi^{2}=\Pi, \quad\langle\Pi\rangle=\boldsymbol{e}^{*} \boldsymbol{e}=1,
$$

is projection operator for the determination of non-degenerate solutions of Equations (10.1). This explains the last part of the formulae (10.8) for the group velocity (taking into account the general identity $\langle(\boldsymbol{a} \cdot \boldsymbol{b}) \mathrm{A}\rangle=\boldsymbol{b} \mathrm{A} \boldsymbol{a})$. In case of $\overline{\mathrm{L}}=0,(\Rightarrow[\mathrm{L}]=0)$, the first and second part of relations (10.8) become indeterminate due to vanishing numerator and denominator but the last part with representation by the electric field amplitudes remains true. This singular case is the case of optic axes or binormals which we do not further discuss here since it leads us far from our proper aim.

With the first or second part of formulae (10.8), one can find explicit formulae for the group velocity which express it as a function of wave vector $\boldsymbol{k}$, frequency $\omega$ and medium properties involved in the permittivity tensor $\boldsymbol{\varepsilon} \equiv \boldsymbol{\varepsilon}(\boldsymbol{k}, \omega)$. Although not difficult to obtain, however, they are long taking into account the dispersion and, therefore, we will not write them down (we give them in next Section under neglect of dispersion). Instead of this we will use last part of (10.8) which reveals interesting relations to the action 4-vector and to the energy-momentum tensor. According to (10.8), the group velocity $\boldsymbol{v}_{0}$ at the considered point $\left(\boldsymbol{k}=\boldsymbol{k}_{0}, \omega=\omega_{0} \equiv \omega\left(\boldsymbol{k}_{0}\right)\right)$ of the dispersion surface (10.5) is determined by

$$
v_{0, l}=-\frac{\boldsymbol{E}_{0}^{*}\left(\frac{\partial \mathrm{L}}{\partial k_{l}}\right)_{0} \boldsymbol{E}_{0}}{\boldsymbol{E}_{0}^{*}\left(\frac{\partial \mathrm{L}}{\partial \omega}\right)_{0} \boldsymbol{E}_{0}}=\frac{T_{l}}{s},
$$

where we used the representation (6.12) for action flow density $\boldsymbol{T}$ and action density $s$ in the limiting case of plane monochromatic waves. The energymomentum tensor for this limiting case of plane monochromatic waves can now be represented in the form (see also next Section)

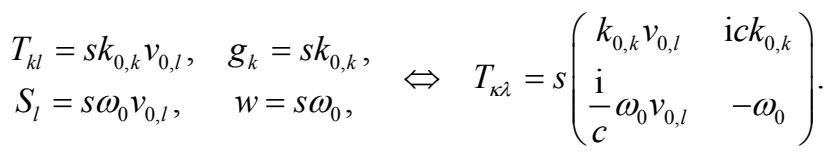


From this it can be easily seen for this limiting case

$$
\mathrm{T}=\boldsymbol{g} \cdot \boldsymbol{v}_{0}, \quad \boldsymbol{S}=w \boldsymbol{v}_{0}, \quad \Rightarrow \quad \frac{T_{k l}}{g_{k}}=\frac{S_{l}}{w}=v_{0, l},
$$

which means that the three-dimensional stress tensor $\mathrm{T}$ which in considered case is a dyadic product is proportional to the momentum density $\boldsymbol{g}$ and the energy flow density $S$ to the energy density $w$ with the group velocity $\boldsymbol{v}_{0}$ as the proportionality factor in analogy to (10.12).

As it is well known [3], the velocity $\boldsymbol{v}_{0}$ is not spatial part of a relativistic covariant 4 -vector but with following modification by the factor

$$
\gamma_{0} \equiv \frac{1}{\sqrt{1-\frac{v_{0}^{2}}{c^{2}}}}
$$

one obtains the relativistic covariant 4 -vector of velocity $u_{0, \lambda}$

$$
u_{0, \lambda} \equiv\left(u_{0, l}, u_{0,4}\right)=\gamma_{0}\left(v_{0, l}, \mathrm{i} c\right), \Rightarrow u_{0}^{2} \equiv u_{0, \lambda} u_{0, \lambda}=-c^{2} .
$$

Using it the energy-momentum tensor (10.13) may be represented in the following relativistic covariant form

$$
T_{\kappa \lambda}=s_{0} k_{0, \kappa} u_{0, \lambda}=s_{0}\left(\begin{array}{cc}
k_{0, k} u_{0, l} & \mathrm{i} \gamma_{0} c k_{0, k} \\
\frac{\mathrm{i}}{c} \omega_{0} u_{0, l} & -\gamma_{0} \omega_{0}
\end{array}\right), \quad s_{0}=s \sqrt{1-\frac{\boldsymbol{v}_{0}^{2}}{c^{2}}} \equiv \frac{s}{\gamma_{0}},
$$

where $s_{0}$ is the action density in the inertial system where the wave packet is resting. This is in analogy to a homogeneous particle flow in classical hydrodynamics without interaction of the particles (or without inner pressure) for which the energy-momentum tensor possesses the form

$$
T_{\kappa \lambda}=n_{0} p_{0, \kappa} u_{0, \lambda}, \quad p_{0, \kappa}=m_{0} u_{0, \kappa}, \quad n_{0}=n \sqrt{1-\frac{v_{0}^{2}}{c^{2}}} \equiv \frac{n}{\gamma_{0}},
$$

where $p_{0, \kappa}=m_{0} u_{0, \kappa}$ is the momentum of one particle, $m_{0}$ its rest mass and $n_{0}$ the particle density in the inertial system where the particles rest (e.g., [12]). The analogy of (10.17) to (10.18) for a homogeneous particle flow suggests (with knowledge of quantum theory) to interpret the first as homogeneous flow of quasiparticles and to introduce an abbreviation $\hbar$ according to

$$
\hbar \equiv \frac{s_{0}}{n_{0}}=\frac{s}{n},
$$

as action of one particle independently of the considered inertial system (i.e., as a Lorentz invariant and, moreover, even as adiabatic Lorentz invariant as may be shown) and we may write

$$
p_{0, \kappa}=\frac{s_{0}}{n_{0}} k_{0, \kappa}=\hbar k_{0, \kappa},
$$

as 4-vector of the momentum of quasiparticles in agreement with quantum theory. Then we have analogous expressions for the energy-momentum tensors of a homogeneous flow without pressure in classical hydrodynamics on one side 
and in macroscopic electrodynamics on the other side where the last provides a richer variety of possible functional dependencies $v \equiv v(k) \quad($ or $v \equiv v(p))$ or of their inversion $\boldsymbol{k} \equiv \boldsymbol{k}(\boldsymbol{v}) \quad($ or $\boldsymbol{p} \equiv \boldsymbol{p}(\boldsymbol{v})$ ) than (relativistic) classical mechanics which considers only

$$
\underset{=\hbar k}{p} \equiv \underbrace{\left(\boldsymbol{p}, \mathrm{i} \frac{E}{c}\right)}_{=\hbar\left(\boldsymbol{k}, \mathrm{i} \frac{\omega}{c}\right)}=m_{0}\left(\frac{\boldsymbol{v}}{\sqrt{1-\frac{\boldsymbol{v}^{2}}{c^{2}}}}, \mathrm{i} \frac{c}{\sqrt{1-\frac{\boldsymbol{v}^{2}}{c^{2}}}}\right) \equiv m_{0} u,
$$

with $m_{0}$ the rest mass of one particle. Usually, the relation between 4 -vectors $p$ and $u$ in macroscopic electrodynamics is a 4-tensorial one with tensor components depending on components of $v$ separately where this cannot be expressed by only relativistic scalars such as $m_{0}$. A certain exception is formed by transverse waves in a cold isotropic plasma (Section 12).

It was mentioned but not explicitly shown that the local action conservation (6.8) or (6.11) is a more general conservation law than the local energy-momentum conservation (7.2) or (8.2) together with (8.3) and holds also for inhomogeneous media (in general, spatially and temporarily inhomogeneous). If we suppose that the action conservation is true for an inhomogeneous medium that means $\nabla_{\lambda} T_{\lambda}(r)=0$ it is informative to see how the energy-momentum conservation is lost for such a medium in case of propagation of almost plane monochromatic waves as here considered. We may assume that in a weakly inhomogeneous medium as main effect the 4 -wave vector $k_{0}$ becomes dependent on the considered space-time point $r \equiv(r, t)$ within the medium that means $k_{0}=k_{0}(r)$. Then we find for the 4-divergence of the energy-momentum tensor under the supposition that local action conservation $\nabla_{\lambda} T_{\lambda}(r)=0$ holds

$$
\begin{aligned}
\nabla_{\lambda} T_{\kappa \lambda}(r) & =\nabla_{\lambda}\left(k_{0, \kappa}(r) T_{\lambda}(r)\right) \\
& =\frac{\partial k_{0, \kappa}}{\partial r_{\lambda}}(r) \underbrace{T_{\lambda}(r)}_{=s_{0}(r) u_{0, \lambda}(r)}+k_{0, \kappa}(r) \underbrace{\nabla_{\lambda} T_{\lambda}(r)}_{=0} \\
& =\frac{\partial k_{0, \kappa}}{\partial r_{\lambda}}(r) T_{\lambda}(r) .
\end{aligned}
$$

The right-hand side is non-vanishing that corresponds to local non-conservation of energy-momentum and the 4-divergence of the energy-momentum tensor (if we overtake its formula from the homogeneous medium) becomes a linear combination of the components of the action vector $T_{\lambda}(r)$.

\section{Neglect of Spatial and Frequency Dispersion and Group Velocity}

In the considerations of Section 10 about the group velocity and the representation of the energy-momentum tensor in the limiting case of plane monochromatic waves in analogy to that for a homogeneous particle flow we did not use the explicit form of the determinant $|\mathrm{L}(\boldsymbol{k}, \omega)|$ of the wave-equation operator 
$\mathrm{L}(\boldsymbol{k}, \omega)$. In the following, we will make some explicit calculations of the group velocity under neglect of the dispersion (spatial and frequency one).

Neglecting spatial and temporal dispersion of the medium means that we consider

$$
\boldsymbol{\varepsilon}(\boldsymbol{k}, \omega) \equiv \boldsymbol{\varepsilon}_{0}
$$

as a constant permittivity tensor $\varepsilon_{0}$ in the inertial system of the resting medium or, at least, as a good approximation for a neighborhood of the considered mean wave vector $\boldsymbol{k}_{0}$ and mean frequency $\omega_{0}$. Using the first of the relations for the group velocity in (10.8) and a transformation of the denominator by means of the dispersion equation $|\mathrm{L}(\boldsymbol{k}, \omega)|=0$, we find

$$
\boldsymbol{v}=\omega \frac{2\left(\boldsymbol{k} \varepsilon_{0} \boldsymbol{k}\right) \boldsymbol{k}+\boldsymbol{k}^{2}\left(\varepsilon_{0} \boldsymbol{k}+\boldsymbol{k} \varepsilon_{0}\right)-\frac{\omega^{2}}{c^{2}}\left(\left\langle\varepsilon_{0}\right\rangle\left(\varepsilon_{0} \boldsymbol{k}+\boldsymbol{k} \varepsilon_{0}\right)-\left(\varepsilon_{0}^{2} \boldsymbol{k}+\boldsymbol{k} \varepsilon_{0}^{2}\right)\right)}{4 \boldsymbol{k}^{2}\left(\boldsymbol{k} \varepsilon_{0} \boldsymbol{k}\right)-2 \frac{\omega^{2}}{c^{2}}\left(\left\langle\boldsymbol{\varepsilon}_{0}\right\rangle \boldsymbol{k} \varepsilon_{0} \boldsymbol{k}-\boldsymbol{k} \varepsilon_{0}^{2} \boldsymbol{k}\right)},
$$

where we emphasize that the permittivity tensor $\varepsilon_{0}$ herein is, in general, not a symmetric tensor that includes gyrotropy of the medium (see also (4.5)). Furthermore, in general, the directions of $\boldsymbol{k}$ and $\boldsymbol{v}$ in anisotropic media are different. From (11.2) follows immediately for the scalar product of wave vector with group velocity

$$
\boldsymbol{k} \boldsymbol{v}=\omega,
$$

that proves to be equivalent to vanishing of the trace of the energy-momentum tensor under neglect of dispersion (see (9.9) in connection with (9.7) and (10.12)). According to (15) this also means that the frequency $\omega^{\prime}$ in the inertial system $\mathcal{I}^{\prime}=\mathcal{I}_{0}$ which moves with the group velocity $\boldsymbol{v}$ in the inertial system $\mathcal{I}$ of the resting medium (i.e. $\boldsymbol{V}=\boldsymbol{v}$ ) vanishes and due to (A.17) that the wave vector is transformed in the following way $\left(\gamma^{2} \equiv\left(1-\frac{v^{2}}{c^{2}}\right)^{-1}\right)$

$$
\omega_{0}^{\prime}=0, \quad\left[\boldsymbol{k}_{0}^{\prime}, \boldsymbol{v}\right]=[\boldsymbol{k}, \boldsymbol{v}], \quad \boldsymbol{k}_{0}^{\prime} \boldsymbol{v}=\gamma\left(\boldsymbol{k} \boldsymbol{v}-\omega \frac{\boldsymbol{v}^{2}}{c^{2}}\right)=\gamma \omega\left(1-\frac{\boldsymbol{v}^{2}}{c^{2}}\right)=\frac{\omega}{\gamma} .
$$

However, already the presence of frequency dispersion (and, moreover, of spatial dispersion) destroys these relations since we have then additional terms in the denominator of the right-hand side in (11.2) which contain the derivatives $\frac{\partial \boldsymbol{\varepsilon}}{\partial \omega}$ of the permittivity tensor $\boldsymbol{\varepsilon} \equiv \boldsymbol{\varepsilon}(\omega)$.

In case of neglected dispersion, the operator $\mathrm{L}(\boldsymbol{k}, \omega)$ becomes a function of only a vector $\boldsymbol{n}$ which is called refraction vector (and, clearly, of medium properties contained in $\varepsilon_{0}$ ) according to

$$
\mathrm{L}(\boldsymbol{k}, \omega) \rightarrow \mathrm{L}(\boldsymbol{n}) \equiv \boldsymbol{n} \cdot \boldsymbol{n}-\boldsymbol{n}^{2} \mathrm{I}+\boldsymbol{\varepsilon}_{0}, \quad \boldsymbol{n} \equiv \frac{c}{\omega} \boldsymbol{k} .
$$

The dispersion equation $|\mathrm{L}(\boldsymbol{n})|=0$ leads to the following fourth-order equation in the components of the refraction vector $n$ (e.g., [9], Eq. (2.22)) 


$$
|\mathrm{L}(\boldsymbol{n})|=\left(\boldsymbol{n}^{2}-\left\langle\boldsymbol{\varepsilon}_{0}\right\rangle\right) \boldsymbol{n} \boldsymbol{\varepsilon}_{0} \boldsymbol{n}+\boldsymbol{n} \boldsymbol{\varepsilon}_{0}^{2} \boldsymbol{n}+\left|\boldsymbol{\varepsilon}_{0}\right|=0
$$

The dispersion surface describes now a two-dimensional surface in the three-dimensional space of refraction vectors $\boldsymbol{n}$ which is better accessible for visualization than the three-dimensional (hyper-) surface in four-dimensional $(\boldsymbol{k}, \omega)$-space described by Equation (10.3).

Under neglect of the dispersion, the group velocity $v$ depends only on the quotient $\frac{\boldsymbol{k}}{\omega}$ as one can explicitly see from (11.2) and, therefore, on the refraction vector $\frac{c}{\omega} \boldsymbol{k} \equiv \boldsymbol{n}$. It is favorable to normalize the group velocity by the light velocity $c$ and to introduce together with the refraction vector $\boldsymbol{n}$ a ray vector $\boldsymbol{s}$ by (our notations agree with that of Landau and Lifshits, Vol. VIII [7]) ${ }^{12}$

$$
\boldsymbol{n} \equiv \frac{c \boldsymbol{k}}{\omega}, \quad \boldsymbol{s} \equiv \frac{\boldsymbol{v}}{c}, \quad \Leftrightarrow \quad \boldsymbol{k} \equiv \frac{\omega}{c} \boldsymbol{n}, \quad \boldsymbol{v} \equiv c \boldsymbol{s},
$$

and from (11.3) follows

$$
n s=1 .
$$

If we substitute now the operator $\mathrm{L}(\boldsymbol{k}, \omega)$ by the operator $\mathrm{L}(\boldsymbol{n})$ according to $\mathrm{L}(\boldsymbol{k}, \omega) \rightarrow \mathrm{L}(\boldsymbol{n})$, then we have to substitute derivatives of these operators and of their functions according to $\frac{\partial}{\partial k_{l}} \rightarrow \frac{\partial n_{k}}{\partial k_{l}} \frac{\partial}{\partial n_{k}}=\frac{c}{\omega} \frac{\partial}{\partial n_{l}}$,

$\frac{\partial}{\partial \omega} \rightarrow \frac{\partial n_{k}}{\partial \omega} \frac{\partial}{\partial n_{k}}=-\frac{n_{k}}{\omega} \frac{\partial}{\partial n_{k}}$. Applied to the ray vector $\boldsymbol{s} \equiv \frac{\boldsymbol{v}}{c}$ this means that formula (10.8) can now be substituted by

$$
\boldsymbol{s}=\frac{\frac{\partial|\mathrm{L}|}{\partial \boldsymbol{n}}}{n_{k} \frac{\partial|\mathrm{L}|}{\partial n_{k}}}=\frac{\left\langle\overline{\mathrm{L}} \frac{\partial \mathrm{L}}{\partial \boldsymbol{n}}\right\rangle}{n_{k}\left\langle\overline{\mathrm{L}} \frac{\partial \mathrm{L}}{\partial n_{k}}\right\rangle}=\frac{\boldsymbol{E}_{0}^{*} \frac{\partial \mathrm{L}}{\partial \boldsymbol{n}} \boldsymbol{E}_{0}}{n_{k} \boldsymbol{E}_{0}^{*} \frac{\partial \mathrm{L}}{\partial n_{k}} \boldsymbol{E}_{0}} .
$$

where vectorial indices of $s$ and of $\frac{\partial}{\partial \boldsymbol{n}}$ in numerator correspond to each other (or $s_{l}=\frac{\boldsymbol{E}_{0}^{*} \frac{\partial \mathrm{L}}{\partial n_{l}} \boldsymbol{E}_{0}}{n_{k} \boldsymbol{E}_{0}^{*} \frac{\partial \mathrm{L}}{\partial n_{k}} \boldsymbol{E}_{0}}$ and $\left.\boldsymbol{E}_{0}^{*} \frac{\partial \mathrm{L}}{\partial n_{l}} \boldsymbol{E}_{0} \equiv E_{0, i}^{*} \frac{\partial L_{i j}}{\partial n_{l}} E_{0, j}\right)$. The first part of this equation shows the well-known property that in case of neglected dispersion the ray vector is proportional to the gradient $\frac{\partial|\mathrm{L}|}{\partial \boldsymbol{n}}$ of the dispersion surface $|\mathrm{L}(\boldsymbol{n})|=0$ at the considered point and the denominator determines its norma-

\footnotetext{
${ }^{12}$ Many authors, however, denote with $\boldsymbol{n}$ a unit vector in direction of $\boldsymbol{k}$, for example, Fyodorov [37] [38], the initiator of coordinate-invariant methods, who denotes refraction vectors by $\boldsymbol{m}$ and ray vectors by $p$ and, furthermore, denotes with $s$ a unit vector in direction of the ray vector. Born and Wolf [47] denote with $s$ a unit vector in direction of the wave vector $\boldsymbol{k}$ and with $\boldsymbol{t}$ a unit vector in direction of the ray vector. In our notation we have conveniently $\boldsymbol{n}^{2}=n^{2}$ where $n \equiv|n|$ is the index of refraction of the wave.
} 
lization according to $\boldsymbol{n s}=1$. With the explicit form of the determinant $|\mathrm{L}(\boldsymbol{n})|$ given in (11.6) from which we easily calculate its derivatives with respect to the refraction vector $\boldsymbol{n}$ we find for the ray vector

$$
\boldsymbol{s}=\frac{2\left(n \varepsilon_{0} n\right) n+\left(n^{2}-\left\langle\varepsilon_{0}\right\rangle\right)\left(n \varepsilon_{0}+\varepsilon_{0} n\right)+\varepsilon_{0}^{2} n+n \varepsilon_{0}^{2}}{2\left(\left(2 n^{2}-\left\langle\varepsilon_{0}\right\rangle\right) n \varepsilon_{0} n+n \varepsilon_{0}^{2} n\right)} \equiv s(n),
$$

confirmed by the calculation from (11.2) using the definition of the ray vector. However, the main purpose of these calculations was to establish the algebraic structure of the ray vectors $s$ that means its connection to the invariants of $\mathrm{L} \equiv \mathrm{L}(\boldsymbol{n})$ and to its complementary operator $\overline{\mathrm{L}}$. The inversion of the vectorial function $\boldsymbol{s}=\boldsymbol{s}(\boldsymbol{n})$ to a vectorial function $\boldsymbol{n}=\boldsymbol{n}(\boldsymbol{s})$ by means of duality relations between ray and refraction quantities or otherwise is up to now only solved under the restriction $\boldsymbol{\varepsilon}_{0}=\boldsymbol{\varepsilon}_{0}^{\mathrm{T}}$ that means to nongyrotropy of the medium.

Using the refraction vector $\boldsymbol{n}$ and the ray vector $s$, the 4-wave vector $k$ and the 4 -vector of velocity $u$ (see (10.16)) can be represented as follows

$$
\begin{aligned}
& k \equiv\left(\boldsymbol{k}, \mathrm{i} \frac{\omega}{c}\right)=\frac{\omega}{c}(\boldsymbol{n}, \mathrm{i}), \Rightarrow k^{2}=\boldsymbol{k}^{2}-\frac{\omega^{2}}{c^{2}}=\frac{\omega^{2}}{c^{2}}\left(\boldsymbol{n}^{2}-1\right), \\
& u \equiv\left(\frac{\boldsymbol{v}}{\sqrt{1-\frac{\boldsymbol{v}^{2}}{c^{2}}}}, \mathrm{i} \frac{c}{\sqrt{1-\frac{\boldsymbol{v}^{2}}{c^{2}}}}\right)=c\left(\frac{\boldsymbol{s}}{\sqrt{1-\boldsymbol{s}^{2}}}, \mathrm{i} \frac{1}{\sqrt{1-\boldsymbol{s}^{2}}}\right), \Rightarrow u^{2}=-c^{2} .
\end{aligned}
$$

The scalar product of 4-wave vector and 4-velocity becomes vanishing in case of neglected dispersion

$$
k u=\frac{\boldsymbol{k} \boldsymbol{v}-\omega}{\sqrt{1-\frac{\boldsymbol{v}^{2}}{c^{2}}}}=\omega \frac{\boldsymbol{n s}-1}{\sqrt{1-\boldsymbol{s}^{2}}}=0 .
$$

The scalar products of 4 -vectors $k^{2}, u^{2}$ and $k u$ are Lorentz invariants and values for them which are calculated in one inertial system such as here in the inertial system of the resting medium remain the same in arbitrary other inertial systems.

If we apply this to the mean wave vector $\boldsymbol{k}_{0}$ and mean frequency $\omega_{0}$ in the expressions for the energy-momentum tensor in first approximation in (9.7), we find for neglected dispersion

$$
T_{\kappa \kappa}=T_{k k}-w=0, \Leftrightarrow\langle\mathrm{T}\rangle \equiv T_{k k}=w,
$$

which means that the trace of the energy-momentum tensor $T_{\kappa \lambda}$ is vanishing in such approximation. This is well known for the general energy-momentum tensor in vacuum electrodynamics [3]. Using this together with (9.11) we find for neglected dispersion

$$
\langle\mathrm{T}\rangle=w, \quad \boldsymbol{S g}=\langle\mathrm{T}\rangle w \Rightarrow \boldsymbol{S g}=\langle\mathrm{T}\rangle^{2}=w^{2} \geq 0 .
$$

This means, in particular, that the scalar product of energy flow density $\boldsymbol{S}$ with momentum density $\boldsymbol{g}$ is equal to the square of the energy density $w$. 
Furthermore, under neglect of dispersion and in approximation of plane monochromatic waves, we find from (9.8)

$$
k_{0, \lambda} T_{\lambda}=k_{0, l} T_{l}-\omega_{0} s=0, \quad \Rightarrow \quad k_{0, l} T_{k l}=\omega_{0} g_{k}, \quad k_{0, l} S_{l}=\omega_{0} w,
$$

that, however, is no more true taking into account the dispersion of the medium. First relation in (11.15) states that the scalar product $k_{0, \lambda} T_{\lambda}$ of mean 4 -wave vector $k_{0, \lambda}$ with action 4 -vector $T_{\lambda}$ vanishes meaning that they are mutually orthogonal in Minkowski space. Last relation in (11.14) suggests that the scalar product of energy flow density $\boldsymbol{S}$ with momentum density $\boldsymbol{g}$ should be greater than zero or otherwise these two vectors form an angle greater than $\frac{\pi}{2}$.

As a special case we consider now transversal waves in a resting isotropic medium under neglect of dispersion with the constant permittivity tensor $\varepsilon_{0, i j}=\varepsilon_{0} \delta_{i j}$ for which we find the following relations between wave vector $\boldsymbol{k}$ and group velocity $v$

$$
\boldsymbol{\varepsilon}=\varepsilon_{0} I, \Rightarrow \boldsymbol{k}^{2}=\frac{\omega^{2}}{c^{2}} \varepsilon_{0}, \quad \boldsymbol{v}=\frac{c^{2}}{\omega \varepsilon_{0}} \boldsymbol{k}=\frac{c}{\sqrt{\varepsilon_{0}}} \frac{\boldsymbol{k}}{|\boldsymbol{k}|}, \quad \boldsymbol{k} \boldsymbol{v}-\omega=0,
$$

and between ray vector $s$ and refraction vector $n$

$$
\boldsymbol{s} \equiv \frac{\boldsymbol{v}}{c}=\frac{c \boldsymbol{k}}{\omega \varepsilon_{0}} \equiv \frac{\boldsymbol{n}}{\varepsilon_{0}}, \Leftrightarrow k \equiv\left(\boldsymbol{k}, i \frac{\omega}{c}\right)=\frac{\omega \varepsilon_{0}}{c^{2}}\left(\boldsymbol{v}, i \frac{c}{\varepsilon_{0}}\right) .
$$

For the energy-momentum tensor then follows according to (10.13)

$$
\begin{aligned}
T(r) & \equiv\left(\begin{array}{cc}
\mathrm{T} & \mathrm{i} c \boldsymbol{g} \\
\frac{\mathrm{i}}{c} \boldsymbol{S} & -w
\end{array}\right)=s(r)\left(\begin{array}{ll}
\boldsymbol{k} \cdot \boldsymbol{v} & \mathrm{i} c \boldsymbol{k} \\
\frac{\mathrm{i}}{c} \omega \boldsymbol{v} & -\omega
\end{array}\right) \\
& =s(r) \omega\left(\begin{array}{cc}
\varepsilon_{0} \boldsymbol{s} \cdot \boldsymbol{s}, & \mathrm{i} \varepsilon_{0} \boldsymbol{s} \\
\mathrm{i} \boldsymbol{s} & -1
\end{array}\right)=s(r) \frac{\omega}{\varepsilon_{0}}\left(\begin{array}{cc}
\boldsymbol{n} \cdot \boldsymbol{n} & \mathrm{i} \varepsilon_{0} \boldsymbol{n} \\
\mathrm{i} \boldsymbol{n} & -\varepsilon_{0}
\end{array}\right),
\end{aligned}
$$

where $s(r)$ is the action density in the system of the resting medium (in contrast to ray vector $s)$. This tensor is non-symmetric but its spatial part $\mathrm{T}=s(r) \boldsymbol{k} \cdot \boldsymbol{v}$ is symmetric since the vectors $\boldsymbol{k}$ and $\boldsymbol{v}$ possess the same direction (for the necessity of this symmetry for isotropic media see Section 14). The trace $\langle T\rangle$ of the four-dimensional tensor $T$ is vanishing

$$
\langle T(r)\rangle=s(r)(\boldsymbol{k} v-\omega)=0,
$$

that is only true under neglect of the dispersion. In the next Section we discuss the energy-momentum tensor for a special isotropic medium but without neglect of the dispersion.

\section{Energy-Momentum Tensor of a Cold Isotropic Plasma and Transverse Photons with Scalar Rest Mass}

As one of the simplest models including frequency dispersion in explicit form we now consider a cold isotropic plasma. Its energy-momentum tensor shows the peculiarity that it is symmetric. Without an external magnetic field it pos- 
sesses the following permittivity tensor, e.g., [7] [11]

$$
\varepsilon_{i j}(\boldsymbol{k}, \omega)=\varepsilon(\omega) \delta_{i j}, \quad \varepsilon(\omega)=1-\frac{\omega_{p}^{2}}{\omega^{2}}, \quad \omega_{p} \equiv \sqrt{\frac{4 \pi n_{e} e^{2}}{m_{e}}},
$$

where $\omega_{p}$ denotes the plasma frequency which, e.g., for an electron plasma is expressed expressed by the plasma parameters $n_{e}, e, m_{e}$ (electron density, electron charge and electron mass).

The dispersion equation $\varepsilon(\omega)=0$ for longitudinal waves with its resolution $\omega=\omega_{p}$ does not include the wave vector $\boldsymbol{k}$ and therefore the group velocity $v$ vanishes. This means that longitudinal localized excitations cannot propagate or decay in the approximation of absent spatial dispersion and absent losses. The energy-momentum tensor for these excitations can be obtained from (10.13) by setting $\boldsymbol{v}_{0}=\mathbf{0}$ for the group velocity and using relation (10.19) between action and particle (excitation) density.

The dispersion equation for transverse waves $c^{2} \boldsymbol{k}^{2}=\omega^{2} \varepsilon(\omega)$ with real wave vector $\boldsymbol{k}=\boldsymbol{k}^{*}$ is

$$
c^{2} \boldsymbol{k}^{2}-\omega^{2}+\omega_{p}^{2}=0, \Rightarrow \frac{\omega}{\omega_{p}}=\frac{\sqrt{\omega_{p}^{2}+c^{2} \boldsymbol{k}^{2}}}{\omega_{p}} \geq 1 .
$$

From (12.2) follows for the group velocity $v$ of transverse waves or of their quasiparticles

$$
\boldsymbol{v} \equiv \frac{\partial \omega}{\partial \boldsymbol{k}}=\frac{c^{2} \boldsymbol{k}}{\omega}=c \sqrt{\frac{c^{2} \boldsymbol{k}^{2}}{\omega_{p}^{2}+c^{2} \boldsymbol{k}^{2}}} \frac{\boldsymbol{k}}{|\boldsymbol{k}|} \equiv \boldsymbol{v}(\boldsymbol{k}), \Rightarrow \frac{|\boldsymbol{v}|}{c}=\sqrt{\frac{c^{2} \boldsymbol{k}^{2}}{\omega_{p}^{2}+c^{2} \boldsymbol{k}^{2}}} \leq 1,
$$

with $|\boldsymbol{k}| \equiv \sqrt{\boldsymbol{k}^{2}}$ and $|\boldsymbol{v}| \equiv \sqrt{\boldsymbol{v}^{2}}$. Due to isotropy, the group velocity $\boldsymbol{v}$ possesses the direction of the wave vector $\boldsymbol{k}$ and $\boldsymbol{v} \equiv \boldsymbol{v}(\boldsymbol{k})$ can be converted to the vectorial function $\boldsymbol{k} \equiv \boldsymbol{k}(\boldsymbol{v})$ according to

$$
\boldsymbol{k}=|\boldsymbol{k}| \frac{\boldsymbol{v}}{|\boldsymbol{v}|}=\frac{\omega_{p}}{c^{2}} \frac{\boldsymbol{v}}{\sqrt{1-\frac{\boldsymbol{v}^{2}}{c^{2}}}} \equiv \boldsymbol{k}(\boldsymbol{v}), \Rightarrow \boldsymbol{k} \boldsymbol{v}=\omega \frac{\boldsymbol{v}^{2}}{c^{2}}, \quad \frac{c^{2} \boldsymbol{k}^{2}}{\omega_{p}^{2}}=\frac{\frac{\boldsymbol{v}^{2}}{c^{2}}}{1-\frac{\boldsymbol{v}^{2}}{c^{2}}} .
$$

We introduce an abbreviation $\gamma$ in analogy to the procedure for special Lorentz transformations by

$$
\gamma \equiv \frac{1}{\sqrt{1-\frac{\boldsymbol{v}^{2}}{c^{2}}}}=\frac{\sqrt{\omega_{p}^{2}+c^{2} \boldsymbol{k}^{2}}}{\omega_{p}} \geq 1, \Rightarrow \frac{\boldsymbol{v}^{2}}{c^{2}}=\frac{\gamma^{2}-1}{\gamma^{2}}, \quad \boldsymbol{k} \boldsymbol{v}-\omega=-\frac{\omega_{p}}{\gamma}
$$

which is equivalent to $\omega=\gamma \omega_{p}$ with $\omega_{p}$ the minimal possible frequency for velocity $\boldsymbol{v}=\mathbf{0}$.

The second-order derivatives of $\omega \equiv \omega(\boldsymbol{k})$ for transverse waves in a cold plasma consist of a transverse part proportional to $\delta_{k l}-\frac{k_{k} k_{l}}{|\boldsymbol{k}|^{2}}$ and a longitudinal part proportional to $\frac{k_{k} k_{l}}{|\boldsymbol{k}|^{2}}$ given by 


$$
\frac{\partial^{2} \omega}{\partial k_{k} \partial k_{l}}=\frac{c^{2}}{\omega}\left(\delta_{k l}-\frac{k_{k} k_{l}}{|\boldsymbol{k}|^{2}}+\frac{\omega_{p}^{2}}{\omega^{2}} \frac{k_{k} k_{l}}{|\boldsymbol{k}|^{2}}\right) .
$$

They are responsible for diffraction perpendicular and parallel to the direction of propagation and we call $\frac{\partial^{2} \omega}{\partial k_{k} \partial k_{l}}$ the diffraction tensor.

For the 4-wave vector $k$ we find from (12.4) the following proportionality to the 4-velocity $u$

$$
k \equiv\left(\boldsymbol{k}, \mathrm{i} \frac{\omega}{c}\right)=\frac{\omega_{p}}{c^{2}}(\gamma \boldsymbol{v}, \mathrm{i} \gamma c)=\frac{\omega_{p}}{c^{2}}(\boldsymbol{u}, \mathrm{i} c) \equiv \frac{m_{p}}{\hbar} u,
$$

where we defined a "rest" mass $m_{p}$ which is independent on the velocity $\boldsymbol{v}$ of the moving particles (plasmons) and depends beside physical constants only on the plasma frequency $\omega_{p}$ by

$$
m_{p} c^{2} \equiv \hbar \omega_{p}
$$

From (12.7) follows for the squared modulus $k^{2}$ of the 4-wave vector $k$ which is a Lorentz-invariant

$$
k^{2}=\boldsymbol{k}^{2}-\frac{\omega^{2}}{c^{2}}=-\frac{\omega_{p}^{2}}{c^{2}}=-\frac{m_{p}^{2} c^{2}}{\hbar^{2}},
$$

independently of the frequency $\omega$ in the resting plasma. Therefore, it can also serve as a model for only one kind of transverse photons with the same rest mass independently on the frequency and it seems to be clear that it is the only case of a medium which provide this. Furthermore, we find

$$
k u=\gamma(\boldsymbol{k} \boldsymbol{v}-\omega)=-\omega_{p}=-\frac{m_{p} c^{2}}{\hbar},
$$

in contrast to its vanishing (11.12) in case of neglected dispersion.

For the energy-momentum tensor in three-dimensional representation and written in coordinate-invariant way, i.e., without indices (see Appendix B) and furthermore if we omit the indices " 0 " at $\boldsymbol{k}$ and $\omega_{0}$ we find from (12.3) the relation $\boldsymbol{k}=\frac{\omega}{c^{2}} \boldsymbol{v}=\frac{\omega_{p}}{c^{2}} \boldsymbol{u}$ and using the definition of $m_{p}$ by (12.8)

$$
T \equiv\left(\begin{array}{cc}
\mathrm{T} & \mathrm{i} c \boldsymbol{g} \\
\frac{\mathrm{i}}{c} \boldsymbol{S} & -w
\end{array}\right)=s_{0}\left(\begin{array}{ll}
\boldsymbol{k} \cdot \boldsymbol{v} & \mathrm{i} c \boldsymbol{k} \\
\frac{\mathrm{i}}{c} \omega \boldsymbol{v} & -\omega
\end{array}\right)=n_{0} m_{p}\left(\begin{array}{cc}
\boldsymbol{u} \cdot \boldsymbol{u} & \mathrm{i} c \boldsymbol{u} \\
\mathrm{i} c \boldsymbol{u} & -c^{2}
\end{array}\right),
$$

where $s_{0}=n_{0} \hbar$ is the action density and $n_{0}$ the particle density with mass $m_{p}$ defined in (12.8) in the inertial system $\mathcal{I}_{0}$ of the resting plasma. The four-dimensional energy-momentum tensor (12.11) is completely symmetric and can be represented in the factorized form

$$
T_{\kappa \lambda}=n_{0} m_{p} u_{\kappa} u_{\lambda}=\frac{n_{0}}{m_{p}} \hbar^{2} k_{\kappa} k_{\lambda}=T_{\lambda \kappa},
$$

Its trace is is represented by (see Appendix B) 


$$
\langle T\rangle=\langle\mathrm{T}\rangle-w=s_{0}(\boldsymbol{k} \boldsymbol{v}-\omega)=-s_{0} \frac{\omega_{p}}{\gamma}=-n_{0} \frac{m_{p} c^{2}}{\gamma} \leq 0 .
$$

with the trace of the three-dimensional part of the stress tensor $\mathrm{T}$

$$
\langle\mathrm{T}\rangle=s_{0} \boldsymbol{k} \boldsymbol{v}=\left(\sqrt{n_{0} m_{p}} \gamma \boldsymbol{v}\right)^{2} \geq 0, \quad w=s_{0} \omega \geq 0 .
$$

Since the four-dimension tensor (or operator) $T$ as well as its spatial part $\mathrm{T}$ factorize they possess only one non-vanishing eigenvalue and we have

$$
[T]=0, \quad|T|=0, \quad\|T\|=0, \quad[\mathrm{~T}]=0, \quad|\mathrm{~T}|=0,
$$

From (B.8) follows then

$$
\boldsymbol{S g}=\langle\mathrm{T}\rangle w \geq 0, \quad \boldsymbol{S} \mathrm{T} \boldsymbol{g}=\langle\mathrm{T}\rangle^{2} w \geq 0, \quad \boldsymbol{S} \mathrm{T}^{2} \boldsymbol{g}=\langle\mathrm{T}\rangle^{3} w \geq 0 .
$$

The derivations were made for real wave vector $\boldsymbol{k}$ and since $\boldsymbol{S}$ and $\boldsymbol{g}$ are proportional to $\boldsymbol{k}$ in this case the positivity of $\boldsymbol{S g}$ is also understandable from this side.

In the inertial system $\mathcal{I}^{\prime}$ where the excitation rests that means which moves with group velocity $\boldsymbol{v}$ in $\mathcal{I}_{0}$ and thus where $\boldsymbol{v} \rightarrow \boldsymbol{v}^{\prime}=\mathbf{0}$ we have the energy momentum tensor

$$
\begin{gathered}
T^{\prime}=\left(\begin{array}{cc}
0 & 0 \\
0 & -w^{\prime}
\end{array}\right)=s_{0}^{\prime}\left(\begin{array}{cc}
0 & 0 \\
0 & -\omega^{\prime}
\end{array}\right)=n_{0}^{\prime}\left(\begin{array}{cc}
0 & 0 \\
0 & -m_{p} c^{2}
\end{array}\right), \\
w^{\prime}=n_{0} m_{p} c^{2}=n_{0} \hbar \omega_{p}=s_{0} \omega_{p}, \quad n_{0}^{\prime}=\gamma n_{0},\left\langle T^{\prime}\right\rangle=-w^{\prime} .
\end{gathered}
$$

In the transition $\mathcal{I} \rightarrow \mathcal{I}^{\prime}=\mathcal{I}_{0}$ to this system, we have to transform (see transformation formulae in Appendix A with $\boldsymbol{V}=\boldsymbol{v}): \boldsymbol{k} \rightarrow \boldsymbol{k}^{\prime}=\mathbf{0}, \omega \rightarrow \omega^{\prime}=\omega_{p}$, $\boldsymbol{E}_{0} \rightarrow \boldsymbol{E}_{0}=\sqrt{1-\frac{\boldsymbol{v}^{2}}{c^{2}}} \boldsymbol{E}_{0}$. Therefore, the phase factor $\exp \left\{\mathrm{i}\left(\boldsymbol{k}_{0} \boldsymbol{r}-\omega_{0} t\right)\right\}$ transforms according to

$$
\exp \{\mathrm{i}(\boldsymbol{k} \boldsymbol{r}-\omega t)\} \rightarrow \exp \left\{\mathrm{i}\left(\boldsymbol{k}^{\prime} \boldsymbol{r}^{\prime}-\omega^{\prime} t^{\prime}\right)\right\}=\exp \left(-\mathrm{i} \omega_{p} t^{\prime}\right),
$$

and the excitation appears in $\mathcal{I}^{\prime}$ as a pure oscillation of the electric field in time with plasma frequency $\omega_{p}$ which due to vanishing wave vector cannot be classified as transverse or longitudinal one but is its unification. The specialized formula (A.20) for the susceptibility in the system $\mathcal{I}_{0}$ which moves with group velocity $\boldsymbol{v}_{0}$ of a certain excitation in $\mathcal{I}$ is relatively complicated. The transformation to this system makes only one considered wave to a resting excitation, whereas all other ones are not resting. Therefore, in the system $\mathcal{I}^{\prime}=\mathcal{I}_{0}$ only the excitation with $k_{0}^{\prime}=0, \omega_{0}^{\prime}=\omega_{p}$ is simple, whereas all others remain complicated and propagate with some group velocity.

The action of a cold plasma onto a flow of light particles propagating in vacuum is that it makes them to a flow of transverse quasiparticles and equips the last with a rest mass $m_{p}=\frac{\hbar \omega_{p}}{c^{2}}$. Therefore, it may serve as a concrete model for the transition from massless particles to particles with rest mass. A well-known model for equipping massless particles with a rest mass is the Higgs mechanism 
by symmetry breaking in gauge field theories and this model is similar. The result for the energy-momentum tensor in the limiting case of plane monochromatic waves (12.12) is formulated in Lorentz-covariant form and contains only Lorentz invariants $\left(n_{0}, s_{0}\right.$ are relativistic scalars and $m_{p}$ an invariant). It agrees with the energy-momentum tensor for a homogeneous flow of relativistic point-like particles in classical mechanics (e.g., [3] [5] [12] [15]). In the transition to vacuum $n_{e} \rightarrow 0 \Rightarrow \omega_{p} \rightarrow 0, m_{p} \rightarrow 0$, the rest mass $m_{p}$ goes to zero. In this case we do not have an inertial system where the excitation is resting and the 4-velocity $u_{0, \lambda}$ is diverging due to $\boldsymbol{v}^{2} \rightarrow c^{2}$ and the action density $s_{0}$ in the transition to a resting system goes to zero, i.e. $s_{0} \rightarrow S s_{0}^{\prime}=0$ (see (10.17) and (10.18)). Formula (10.17) for the energy-momentum tensor is then no more applicable but it can be substituted by (10.13) together with the relation $c^{2} \boldsymbol{k}_{0}=\omega_{0} \boldsymbol{v}_{0}$ from (12.3) that leads to the energy-momentum tensor for quasiplane and quasimonochromatic waves in vacuum. The appearance of a rest mass for the elementary transverse excitations in a plasma is a collective effect of the interaction of the charged particles and it vanishes in the transition to vacuum (Lorentz invariance). The cold plasma may serve as orientation for a relativistic covariant electromagnetic theory which provides transverse photons with a certain rest

If we combine formula (12.8) for the rest mass of the quasiparticles with the expression for the plasma frequency of a cold electron plasma in (12.1) then we find

$$
m_{p}=\frac{\hbar \omega_{p}}{c^{2}}=\frac{\hbar}{c^{2}} \sqrt{\frac{4 \pi n_{e} e^{2}}{m_{e}}}=\frac{\hbar}{c} \sqrt{4 \pi n_{e} r_{e}}, \quad\left(r_{e} \equiv \frac{e^{2}}{m_{e} c^{2}}\right)
$$

where $r_{e} \approx 2.82 \times 10^{-13} \mathrm{~cm}$ denotes the classical electron radius. Thus the mass $m_{p}$ is proportional to the square root of the electron density $n_{e}$ but is not in a simple relation to the electron mass $m_{e}$ without taking into account the electron charge $e$. It is proportional to the reciprocal square root of $m_{e}$ if we fix the charge $e$. For plasma frequencies $\omega_{p}$ in the visible region of about $v_{p} \equiv \frac{\omega_{p}}{2 \pi} \approx 5 \times 10^{14} \mathrm{~Hz} \quad\left(\hat{=} \sec ^{-1}\right) \quad$ (for alkali metals they are a little higher and are much higher for most other metals) we find according to (12.8) a mass of about $m_{p} \approx 4 \times 10^{-33} \frac{\mathrm{erg} \cdot \mathrm{sec}^{2}}{\mathrm{~cm}^{2}}=4 \times 10^{-33} \mathrm{~g}$ that is by a factor $\approx 2 \times 10^{5}$ smaller than the rest mass of an electron which is of about $m_{e} \approx 10^{-27} \mathrm{~g}$ corresponding to a rest energy of about $\approx 0.5 \mathrm{MeV}$. Since the appearance of a rest mass is a collective effect (quasi-particles), we cannot separate different parts of energy and momentum from the pure field and from the moving particles (electrons) on the background of the heavier ions considered as resting and making the medium (plasma) macroscopically neutral.

We mention that an energy momentum tensor of the form (12.17) with only one nonvanishing component $T_{44}$ in the energy density part for a point-like resting particle is the starting point for establishing the direct connection of Newton's gravitation law with Einstein's equations of general relativity (see, e.g., 
[3], section 99, Eq. (99.1)).

A warm plasma with spatial inversion as symmetry element possesses a transversal and a longitudinal part of the permittivity tensor proportional to $\delta_{i j}-\frac{k_{i} k_{j}}{\boldsymbol{k}^{2}}$ and to $\frac{k_{i} k_{j}}{\boldsymbol{k}^{2}}$ with parameters $\varepsilon_{t}(|\boldsymbol{k}|, \omega)$ and $\varepsilon_{l}(|\boldsymbol{k}|, \omega)$ and in case of gyrotropy a further term.

\section{Angular Momentum Conservation in Resting Isotropic Media}

The most prominent supporter of the Abraham tensor in old time was Pauli [5] in his younger years. He considers explicitly only isotropic non-dispersive media and it is not clear how these results may be generalized to anisotropic media since no proposal for this exists. In his encyclopedic article published in the age of 21, Pauli [5] sees in electron-theoretical considerations of Abraham a weighty argument in favor of the symmetric Abraham tensor but he discusses also advantages and disadvantages of the Minkowski tensor. Since Pauli's article is about Special and General relativity theory and since the last requires a symmetric energy-momentum tensor as source term in Einstein's equations it is understandable that Pauli looked mainly for arguments in favor of the symmetric Abraham tensor. However, in his late years, apparently, he changed his opinion and favored the non-symmetric Minkowski tensor as the correct one. This can be seen from the supplementary notes made by Pauli in 1956, two years before his death, to the re-edition of his encyclopedic article [5]. In Note 11 with reference to von Laue [14] Pauli praised emphatically the Minkowski tensor as the right one and it seems that he wants to correct his earlier opinion ${ }^{13}$. For anisotropic media which were never explicitly considered by Pauli in this regard it is clear that the energy-momentum tensor cannot be symmetric since the momentum density is in direction of the mean wave vector and the energy-flow density (Poynting vector) in direction of the ray vector which, in general, are not parallel to each other as it is well known from experimental and theoretical crystal optics.

In recent time the Abraham tensor was declared in papers of Leonhardt and coworkers [32] [33] as the correct one. It is easily to conjecture that the same as the young Pauli they want to have a symmetric energy-momentum tensor because the General Relativity theory requires such but they should ask themselves how it can be generalized as such symmetric tensor to general anisotropic media. As mentioned most authors favor the Minkowski tensor as the correct one also for its relativistic covariance but many of them do not consider anisotropic me-

\footnotetext{
${ }^{13}$ The full text of Note 11 in [5] is:" M. v. Laue [see his Relativitätstheorie, Vol. 1 (6th edn., 1955) \$ 19] has shown that only the unsymmetric energy-momentum tensor of Minkowski is correct for a phenomenological description of moving bodies (just as it is in crystals at rest). His argument also emphasizes the validity of the addition theorem of velocities for the ray-velocity (see Eq. (312) of the text), which is in agreement only with this unsymmetric tensor.". The Editors of the Russ. Transl. V.L. Ginzburg and V.P. Frolov make further remarks to this problem with four additional citations, in particular, [16] [17].
} 
dia where the problem becomes more clear though more difficult. The reason for different views to this tensor is different separations of ponderomotive forces in the conservation theorems which in this case do not possess the exact form of local conservation laws. This is discussed in detail by Ginzburg [10]. The discussion of the relations between the Abraham and the Minkowski tensor is usually restricted to isotropic media and we do not know an explicit more general form of the symmetric Abraham tensor for anisotropic dispersive media. For anisotropic media it is evident that the momentum density should possess the direction of the mean wave vector and the energy flow density should be in direction of the group velocity of quasiplane and quasimonochromatic waves which, in general, are different for anisotropic media or, in other case, essential parts of crystal optics would be wrong. This is provided if the momentum density is proportional to $[\boldsymbol{D}, \boldsymbol{B}]$ and the energy flow density proportional to $[\boldsymbol{E}, \boldsymbol{B}]$ as in the Minkowski tensor. Taking into account the dispersion both expression have to be modified as was shown ([9] and Section 9 of present article). In the case of taking into account the dispersion the constitutive relations bring into play additional derivatives of the electric (and in certain cases of the magnetic) field which have to be taken into account in the derivation of conservation laws. Our strategy is to formulate the differential conservation laws without taking into account absorption (dissipation or absorption or even amplification, open system) as exact vanishing of 4-divergences. With dissipation this is impossible. The condition for the permittivity tensor to describe a dissipation-less medium was discussed in Section 3.

Even in the special case of an isotropic medium and under neglect of dispersion (spatial and temporal ones) that means in case of the constitutive relations $\boldsymbol{D}_{0}=\varepsilon_{0} \boldsymbol{E}_{0}, \boldsymbol{D}_{0}^{*}=\varepsilon_{0} \boldsymbol{E}_{0}^{*}$ with constant scalar $\varepsilon_{0}$ the energy-momentum tensor $T_{\kappa \lambda}$ remains, in general, non-symmetric, in particular (see next Section)

$$
T_{k 4}=\mathrm{i} c g_{k}=\mathrm{i} \varepsilon_{0} \frac{S_{k}}{c}=\varepsilon_{0} T_{4 k} \neq T_{4 k},
$$

that is nonsymmetric for $\varepsilon_{0} \neq 1$. However, the stress tensor $T_{k l}$ is symmetric in this case in the inertial system where the isotropic medium is resting (in moving systems it is no more isotropic)

$$
T_{k l}=T_{l k} .
$$

Moreover, this symmetry remains to be true also in the general case of taking into account the dispersion as can be seen from (10.13) and (10.8) since for isotropic media the group velocity $\boldsymbol{v}$ and the wave vector $\boldsymbol{k}$ possess in general case the same direction (see below). This partial symmetry of the three-dimensional part $T_{k l}$ (and only this) of the full four-dimensional energy-momentum tensor $T_{\kappa \lambda}$ is necessary for the existence of a local law of angular-momentum conservation in isotropic media due to invariance with respect to the three-dimensional rotation group in this inertial system where the medium rests.

Multiplying the differential momentum conservation (8.2) by $\varepsilon_{i j k} r_{j} \quad\left(\varepsilon_{i j k}\right.$ is three-dimensional Levi-Civita pseudo-tensor) we may transform this according 
to

$$
0=\varepsilon_{i j k} r_{j} \underbrace{\left.\nabla_{l} T_{k l}+\frac{\partial}{\partial t} g_{k}\right)}_{=0}=\nabla_{l}\left(\varepsilon_{i j k} r_{j} T_{k l}\right)+\frac{\partial}{\partial t}\left(\varepsilon_{i j k} r_{j} g_{k}\right)-\underbrace{\varepsilon_{i j k} T_{k j}}_{=0},
$$

and obtain

$$
\nabla_{l} S_{i l}+\frac{\partial}{\partial t} m_{i}=0, \quad S_{i l} \equiv \varepsilon_{i j k} r_{j} T_{k l}, \quad m_{i} \equiv \varepsilon_{i j k} r_{j} g_{k}=[\boldsymbol{r}, \boldsymbol{g}]_{i},
$$

where we used the symmetry (13.2) and where this last symmetry is evidently required for the vanishing of $\varepsilon_{i j k} T_{k j}$. Herein, $m_{i} \equiv m_{i}(\boldsymbol{r}, t)=[\boldsymbol{r}, \boldsymbol{g}(\boldsymbol{r}, t)]$ means the angular momentum density and $S_{i l} \equiv S_{i l}(\boldsymbol{r}, t)=\varepsilon_{i j k} r_{j} g_{k}(\boldsymbol{r}, t)$ a non-symmetric tensor which is the analogue to the stress tensor for momentum conservation (angular-momentum flow density). Since the momentum density takes into account the polarization of the electromagnetic field the angular momentum density comprises both the orbital and the spin angular momentum.

The vanishing of the right-hand side of (13.4) possesses the form of a differential or local conservation theorem. We also see that a pure translation $\boldsymbol{r} \rightarrow \boldsymbol{r}^{\prime}=\boldsymbol{r}+\boldsymbol{a}$ with a constant vector $\boldsymbol{a}$ (displacement of coordinate origin) does not disturb the local conservation theorem (13.4) although this changes $m_{i}$ and $S_{i l}$. Despite the symmetry (13.2) the whole tensor $T_{\kappa \lambda}$ is non-symmetric $\left(T_{\kappa \lambda} \neq T_{\lambda \kappa}\right)$ due to (13.1) and under Lorentz transformations the spatial part $T_{k l}$ becomes also nonsymmetric $\left(T_{k l}^{\prime} \neq T_{l k}^{\prime}\right)$ as consequence that isotropy of a medium resting in the inertial system $\mathcal{I}$ is lost in inertial systems $\mathcal{I}^{\prime}$ of the moving medium (see Appendix B, in particular, (B.3)). Due to $\varepsilon_{i j k} T_{k j}^{\prime} \neq 0$ a local law of angular-momentum conservation cannot be formulated then in analogy to (13.4) for such inertial systems. One can convert this conclusion. If the four-dimensional energy-momentum tensor $T_{\kappa \lambda}$ would be symmetric in the inertial system of the resting isotropic medium then it remains to be symmetric also in arbitrary other inertial systems and one would be able to prove a local conservation law of angular momentum for an arbitrary inertial system that is evidently wrong (with exception of vacuum). This excludes the symmetric Abraham tensor as candidate for the energy-momentum tensor from the beginning in contradiction to confusing remarks in [5]. We did not find explicit expressions in literature for a symmetric Abraham tensor in the general anisotropic case without or with dispersion where already the same directions of momentum density and energy flow density would be in striking contradiction to known experimental facts.

With exception of the vacuum the angular momentum conservation in isotropic media at rest cannot be extended to a more general four-dimensional conservation theorem such as it was possible for energy and momentum conservation. The reason is that invariance with respect to Lorentz transformations requires a permittivity tensor $\varepsilon_{i j}(\boldsymbol{k}, \omega)=\delta_{i j}$ of the medium which determines the electrodynamic vacuum. We mention that our derivation (13.3) of the necessary symmetry $T_{k l}=T_{l k}$ for local angular momentum conservation for iso- 
tropic media corresponds to the derivation of the necessary symmetry of the four-dimensional tensor $T_{\kappa \lambda}=T_{\lambda \kappa}$ for the local conversation of the four-angular momentum given in [3] (section 32, Eq. 32.10 in Russ. Ed. from 1988).

\section{Non-Uniqueness of the Energy-Momentum Tensor and Its Role for Finding the Simplest One}

As is known the energy-momentum tensor in the local conservation law of energy and momentum (and, similarly, the action 4-vector in local action conservation) is not unique [3]. However, we will suggest that this is not a very strong problem, in particular, not in the approximation of quasiplane and quasimonochromatic waves. We do not strive in this Section for high generality of our considerations and try to illuminate the problems of non-uniqueness only by some remarks.

The general form of non-uniqueness of the energy momentum tensor $T_{\kappa \lambda}(r) \sim T_{\kappa \lambda}^{\prime}(r)$ in the local conservation theorem is described by an arbitrary third-rank four-tensor function $\psi_{\kappa \lambda \mu}(r)$ which is antisymmetric in the last two indices in the following form [3]

$$
T_{\kappa \lambda}^{\prime}(r)=T_{\kappa \lambda}(r)+\nabla_{\mu} \psi_{\kappa \lambda \mu}(r), \quad \psi_{\kappa \lambda \mu}(r)=-\psi_{\kappa \mu \lambda}(r),
$$

from which immediately follows

$$
\nabla_{\lambda} T_{\kappa \lambda}^{\prime}(r)=\nabla_{\lambda} T_{\kappa \lambda}(r)=0 .
$$

In three-dimensional separation this means for the stress tensor $T_{k l}(\boldsymbol{r}, t)$ and the momentum density $g_{k}(\boldsymbol{r}, t)$ and for the energy flow density $S_{l}(\boldsymbol{r}, t)$ and the energy density $w(\boldsymbol{r}, t)$

$$
\begin{aligned}
& T_{k l}^{\prime}(\boldsymbol{r}, t)=T_{k l}(\boldsymbol{r}, t)+\varepsilon_{l m n} \nabla_{m} \psi_{k n}(\boldsymbol{r}, t)+\frac{\partial}{\partial t} \chi_{k l}(\boldsymbol{r}, t), \\
& g_{k}^{\prime}(\boldsymbol{r}, t)=g_{k}(\boldsymbol{r}, t)-\nabla_{l} \chi_{k l}(\boldsymbol{r}, t), \\
& S_{l}^{\prime}(\boldsymbol{r}, t)=S_{l}(\boldsymbol{r}, t)+\varepsilon_{l m n} \nabla_{m} \psi_{n}(\boldsymbol{r}, t)+\frac{\partial}{\partial t} \chi_{l}(\boldsymbol{r}, t), \\
& w^{\prime}(\boldsymbol{r}, t)=w(\boldsymbol{r}, t)-\nabla_{l} \chi_{l}(\boldsymbol{r}, t),
\end{aligned}
$$

with the following separation of $\psi_{\kappa \lambda \mu}(r)$ into arbitrary three-dimensional tensor or pseudo-tensor functions, respectively

$$
\begin{aligned}
& \psi_{k n}(\boldsymbol{r}, t) \equiv \frac{1}{2} \varepsilon_{l m n} \psi_{k l m}(\boldsymbol{r}, t), \quad \chi_{k l}(\boldsymbol{r}, t) \equiv-\frac{\mathrm{i}}{c} \psi_{k l 4}(\boldsymbol{r}, t)=\frac{\mathrm{i}}{c} \psi_{k 4 l}(\boldsymbol{r}, t), \\
& \psi_{n}(\boldsymbol{r}, t) \equiv-\mathrm{i} \frac{c}{2} \varepsilon_{l m n} \psi_{4 l m}(\boldsymbol{r}, t), \quad \chi_{l}(\boldsymbol{r}, t) \equiv-\psi_{4 l 4}(\boldsymbol{r}, t)=\psi_{44 l}(\boldsymbol{r}, t) .
\end{aligned}
$$

For neglected dispersion ( $\varepsilon_{0}$ constant permittivity tensor in $D_{i}=\varepsilon_{0, i j} E_{j}$ ) and without losses $\left(\varepsilon_{0, i j}=\varepsilon_{0, j i}^{*}\right)$ one can derive the following well-known general expressions for the parts of the energy-momentum tensor here denoted by $T_{\kappa \lambda}^{\prime}$ in contrast to our $T_{\kappa \lambda} \quad(\alpha=4 \pi$ in Gauss system)

$$
\begin{aligned}
& \alpha T_{k l}^{\prime}=\frac{1}{2}(\boldsymbol{B B}+\boldsymbol{D} \boldsymbol{E}) \delta_{k l}-\left(B_{k} B_{l}+E_{k} D_{l}\right), \\
& \alpha g_{k}^{\prime}=\frac{1}{c} \varepsilon_{k m n} D_{m} B_{n}=\frac{1}{c}[\boldsymbol{D}, \boldsymbol{B}]_{k},
\end{aligned}
$$




$$
\begin{aligned}
& \alpha S_{l}^{\prime}=c \varepsilon_{l m n} E_{m} B_{n}=c[\boldsymbol{E}, \boldsymbol{B}]_{l}, \\
& \alpha w^{\prime}=\frac{1}{2}(\boldsymbol{B} \boldsymbol{B}+\boldsymbol{D} \boldsymbol{E})=\frac{1}{2}\left(\boldsymbol{B}^{2}+\boldsymbol{E} \boldsymbol{\varepsilon}_{0} \boldsymbol{E}\right),
\end{aligned}
$$

where $\boldsymbol{E}$ and $\boldsymbol{B}$ mean here the full electric and magnetic field and $\boldsymbol{D}$ the full electric induction which depend on $(\boldsymbol{r}, t)$. If we insert into these expressions plane monochromatic waves of the form $\boldsymbol{E}=\boldsymbol{E}_{0} \mathrm{e}^{\mathrm{i}\left(\boldsymbol{k}_{0} r-\omega_{0} t\right)}+\boldsymbol{E}_{0}^{*} \mathrm{e}^{-\mathrm{i}\left(\boldsymbol{k}_{0} r-\omega_{0} t\right)}$ with real $\boldsymbol{k}_{0}$ and $\omega_{0}$ and constant vector amplitudes $\boldsymbol{E}_{0}$ and analogously for $\boldsymbol{B}$ and $\boldsymbol{D}$ we get besides constant terms in the energy-momentum tensor also terms with the rapidly varying phase factors $\mathrm{e}^{\mathrm{ti2}\left(k_{0} r-\omega_{0} t\right)}$. It is not possible to generalize in some simple way the form (14.5) of the energy-momentum tensor containing the full fields to the case of taking into account the dispersion. In particular, the corresponding expressions cannot be local in the fields that means cannot be taken only at the same space-time points $(\boldsymbol{r}, t)$ (see Section 3 ). Therefore, we have to restrict us in the following discussion of the non-uniqueness concerning the terms with rapidly varying phase factors $\mathrm{e}^{ \pm \mathrm{i} 2\left(k_{0} r-\omega_{0} t\right)}$ to the neglect of dispersion.

The suppression of terms with rapidly varying phase factors was made in [34] for local energy conservation (and analogously possible for momentum conservation) in noncovariant form. Here we will show that it can be made also in relativistic covariant form. Under neglect of dispersion and in the limiting case of constant amplitudes $\boldsymbol{E}_{0}$ we can add to our $T_{\kappa \lambda}$ terms with rapidly varying phase factors possessing the form of a four-divergence of a function $\psi_{\kappa \lambda \mu}(r)$ with antisymmetry in last two indices as shown in (14.1) in the following way (recall $\left.k_{0}^{2} \equiv k_{0, \mu} k_{0, \mu}, k_{0} r \equiv k_{0, v} r_{v}\right)$

$$
\begin{aligned}
\alpha T_{\kappa \lambda}^{\prime} & =\alpha T_{\kappa \lambda}+\nabla_{\mu}\left\{\frac{\mathrm{i}}{4 k_{0}^{2}} k_{0, \kappa}\left(\left(\frac{\partial L_{i j}}{\partial k_{\lambda}}\right)_{0} k_{0, \mu}-k_{0, \lambda}\left(\frac{\partial L_{i j}}{\partial k_{\mu}}\right)_{0}\right) E_{0, i} E_{0, j} \mathrm{e}^{\mathrm{i} 2 k_{0} r}+c . c .\right\} \\
& =\alpha T_{\kappa \lambda}-\frac{1}{2 k_{0}^{2}} k_{0, \kappa}\left(\left(\frac{\partial L_{i j}}{\partial k_{\lambda}}\right)_{0} k_{0}^{2}-k_{0, \lambda}\left(\frac{\partial L_{i j}}{\partial k_{\mu}}\right)_{0} k_{0, \mu}\right) E_{0, i} E_{0, j} \mathrm{e}^{\mathrm{i} 2 k_{0} r}+c . c . \\
& =\alpha T_{\kappa \lambda}-\frac{1}{2} k_{0, \kappa}\left(\frac{\partial L_{i j}}{\partial k_{\lambda}}\right)_{0} E_{0, i} E_{0, j} \mathrm{e}^{\mathrm{i} 2 k_{0} r}+\text { c.c. },
\end{aligned}
$$

with $T_{\kappa \lambda}$ denoting the energy-momentum tensor in (9.1) without terms with rapidly varying phase factors and represented by the slowly varying amplitudes

$$
\alpha T_{\kappa \lambda}=-k_{0, \kappa}\left(\frac{\partial L_{i j}}{\partial k_{\lambda}}\right)_{0} E_{0, i}^{*} E_{0, j},
$$

and, in addition, neglecting derivatives $\left(\frac{\partial \varepsilon_{i j}}{\partial k_{\lambda}}\right)_{0}$ in it corresponding to neglect of dispersion. We used in (14.6) the vanishing of the following expression

$$
\begin{aligned}
\left(\frac{\partial L_{i j}}{\partial k_{\mu}}\right)_{0} k_{0, \mu} & =\left(\frac{\partial L_{i j}}{\partial k_{m}}\right)_{0} k_{0, m}-\mathrm{i} c\left(\frac{\partial L_{i j}}{\partial \omega}\right)_{0}\left(\mathrm{i} \frac{\omega_{0}}{c}\right) \\
& =\frac{c^{2}}{\omega_{0}^{2}}\left(k_{0, i} \delta_{j m}+\delta_{i m} k_{0, j}-2 k_{0, m} \delta_{i j}\right) k_{0, m}-2 \frac{c^{2}}{\omega_{0}^{3}}\left(k_{0, i} k_{0, j}-\boldsymbol{k}^{2} \delta_{i j}\right) \omega_{0} \\
& =0
\end{aligned}
$$


which is true for the special form of $L_{i j}$ given in (5.5) only under neglect of dispersion and is related to (11.12) or also to (11.3). Thus we have represented terms with rapidly varying phase factors in the energy-momentum tensor (14.6) by a four-divergence $\nabla_{\mu} \psi_{\kappa \lambda \mu}(r)$ with explicitly given $\psi_{\kappa \lambda \mu}(r)=-\psi_{\kappa \mu \lambda}(r)$ and comparison of (14.6) with the noncovariant form in [30] shows that the choice of $\psi_{\kappa \lambda \mu}(r)$ itself for removing one and the same terms is to certain extent also not fully unique. Evidently, substitutions $\psi_{\kappa \lambda \mu}(r) \rightarrow \psi_{\kappa \lambda \mu}^{\prime}(r)=\psi_{\kappa \lambda \mu}(r)+\nabla_{\nu} \psi_{\kappa \lambda \mu \nu}(r)$ in (14.1) with arbitrary $\psi_{\kappa \lambda \mu \nu}(r)$ which is fully antisymmetric in last three indices $(\lambda, \mu, v)$ provide equivalent possibilities.

Taking into account the dispersion, the expressions (14.5) for the parts of the energy-momentum tensor are no more true. However, one can find from Maxwell equations vanishing quadratic expressions in the field which do not possess the form of local conservation of energy and momentum but are related to it and by averaging these expressions over space and time, we get local conservation laws of energy and momentum which take into account the dispersion in some approximation of quasiplane and quasimonochromatic waves that is demonstrated in [7] for frequency dispersion in the energy density $w$ and energy flow density $\boldsymbol{S}$ where there is obtained the expression for $w$ in (9.7) ( $\boldsymbol{S}$ is not altered in comparison to the usual one due to neglected spatial dispersion). In the derivations of local conservation theorems one cannot work with constant amplitudes and the amplitudes $\boldsymbol{E}_{0}, \boldsymbol{B}_{0}$ and $\boldsymbol{D}_{0}$ are, at least, slowly varying amplitudes. If we take expressions of the kind in (14.6) for removing the terms with rapidly varying phase factors proportional to $\mathrm{e}^{ \pm \mathrm{i} 2\left(k_{0} r-\omega_{0} t\right)}$ then one has also to differentiate the slowly varying amplitudes and there remain some new terms with these phase factors which, however, are small compared with the main terms with these phase factors before. In a second step and successively in higher steps one can try to remove also these smaller terms. However, there is no possibility to remove any parts in the energy-momentum tensor (9.7) which do not contain such rapidly varying phase factors without creating new terms with rapidly varying phase factors or terms which grow in space and time in unreasonable way (e.g., linearly). Clearly, we can derive higher-order approximations of the energy-momentum tensor than in (9.7) (see sections 5-7) and can try to express them not only by the group velocity $v_{l} \equiv \frac{\partial \omega}{\partial k_{l}}$ as done but also by the higher derivatives $\frac{\partial^{2} \omega}{\partial k_{l} \partial k_{m}}, \frac{\partial^{3} \omega}{\partial k_{l} \partial k_{m} \partial k_{n}}$ and so on (this is not yet done) but they also do not contain such rapidly varying phase factors. Therefore, the nonuniqueness cannot be used to make energy-momentum tensors without rapidly varying phase factors to symmetric ones and nonsymmetric energy-momentum tensors of such kind remain intrinsically nonsymmetric. This suggests also that expressions of the kind (9.7) or (9.4) and of their generalization possess some distinguished position with the possibility of direct physical interpretation among all other equivalent energy-momentum tensors in local conservation 
theorems. Our derivations provided these expressions directly without the necessity of suppression of terms by the discussed non-uniqueness.

There is another case where the non-uniqueness of the energy-momentum tensor seems to be of great importance. These are evanescent or inhomogeneous waves with complex wave vector $\boldsymbol{k}_{0}=k_{0}^{\prime}+\mathrm{i} k_{0}^{\prime \prime}$ and (or) complex frequency $\omega_{0}=\omega_{0}^{\prime}+\mathrm{i} \omega_{0}^{\prime \prime}$ leading besides the periodic phase factors $\mathrm{e}^{ \pm \mathrm{i} 2\left(k^{\prime} r-\omega^{\prime} t\right)}$ to exponential factors $\mathrm{e}^{-2\left(\hat{k}_{0}^{\prime \prime} r-\omega_{0}^{\prime \prime} t\right)}$ in the energy-momentum tensor. Such waves are present, for example, under total reflection in the optically thinner medium and in surface waves to both sides of a boundary plane. The exact generalization of the local energy-momentum conservation to such cases is possible. Using the additional factors $\mathrm{e}^{-2\left(k_{0}^{n} r-\omega_{0}^{n} t\right)}$ in such waves provides further possibilities to remove terms in the energy-momentum tensor which are difficult to interpret and to get equivalent tensors but this makes the problems of non-uniqueness more complex.

Summarizing, it seems to us that the non-uniqueness of the energy-momentum tensor can mainly be used to remove or to change terms with periodically or exponentially rapidly changing phase factors, whereas the others are hardly to touch. This problem of non-uniqueness has little to do with the discussion of the correctness of the Minkowski or the Abraham tensor which in absence of dispersion was decided in favor of the Minkowski tensor.

\section{Difficulties for General Relativity Theory Connected with General Asymmetry of Energy-Momentum Tensor in Media}

The energy-momentum tensor $T_{\kappa \lambda}$ forms the source term in Einstein's gravitation equations which determine the metric tensor $g_{\kappa \lambda}$ and thus also the curvature of a Riemannian space-time as a generalization of Minkowski's space-time. Ricci tensor and thus Einstein tensor in these equations are symmetric ones and, consequently, the energy-momentum tensor has also to be symmetric. Since macroscopic electrodynamics is an averaged microscopic electrodynamics it can be assumed that its energy-momentum tensor provides the source term for a correspondingly averaged gravitation field in the medium and requires boundary conditions in case of transition to vacuum with a sharp boundary. The connection of the classical energy-momentum tensor in Minkowski space as source of a curvature in Einstein's equations is patchwork since it starts from a pseudo-Euclidian space but it cannot be fully wrong concerning its symmetry. We will shortly discuss some difficulties which result from this for General relativity theory.

If it would be possible to extend the Ricci tensor in Einstein's equations to a possible non-symmetric one that up to now did not be achieved then, nevertheless, there remain some serious problems. The energy-momentum tensor in the local conservation laws is not uniquely defined (see Section 14) and there arises the problem which of these tensors provides the right source term in Einstein's equations of General relativity theory. Moreover, in spatially and (or) temporally 
inhomogeneous media such a tensor in local conservation laws does not exist at all. In these cases only the local conservation theorem of action remains with a four-dimensional vector of action and action-flow density. One may expect that then this four-vector must be involved in some way as source in generalized Einstein equation but this to our knowledge was also not found up to now.

The unification of the basic laws of physics is a steady desire of physicists. After Einstein's General relativity theory in 1916 it was the problem of its unification with the experimentally well established Maxwell theory of electromagnetism. First successful trials in this direction with extension of the dimensionality of the space-time to 5 dimensions were the Kaluza-Klein theories from about 1920 on. With the foundation of the rigorous quantum theory in about 1925 it became the problem of unification of quantum theory with electromagnetism and gravity from which only the first part found a satisfactory solution in quantum electrodynamics and from this more or less only the microscopic quantum electrodynamics of charged particles is well elaborated. In the sixties and seventies the standard model of elementary particles and fields was established which unified the electromagnetic and the weak with the strong interactions in satisfactory way but with the new problem of symmetry breaking and of the experimental proof of the theoretical Higgs particles. Thus the problem as it represents to us at this time became already the unification of the standard model with a quantum theory of gravitation where great but up to now not fulfilled hopes were set in the development of string theories. The non-symmetry of the energy-momentum tensor for electromagnetic excitations in anisotropic dispersive and, in general, not even homogeneous media in classical electrodynamics as represented here adds a further serious problem because already Einstein's gravitation theory in existing form is not consistent with the electrodynamics of continuous media since first requires a symmetric energy-momentum tensor. This non-symmetry of the energy-momentum tensor is intrinsic and cannot be removed by considering the current and charge distributions of media on the background of the vacuum. Since in the principal correctness of the existing classical electrodynamics of continuous media cannot be doubt the least which is required is some extension or generalization of the General relativity theory if not a more basically new theory. As it seems to us this problem has to be solved before a successful unification with the other fundamental forces of nature can be accomplished.

The General relativity theory has great success for explanation of astronomical observations and for cosmology. It is a beautiful theory which is considered as experimentally verified. It must not be incorrect due to some of the shown classical difficulties and we hope that they can be overcome in the course of time by generalization or somehow in other way and that it remains true as an approximation.

\section{Possible Additions and Generalizations}

The preceding theory of the energy-momentum tensor can be extended in dif- 
ferent directions. In particular, the following possibilities seem to be interesting (partially already elaborated):

1) Statistical model for permittivity tensor for gases and (warm) plasmas with spatial dispersion.

2) Non-statistical permittivity tensor for solids with spatial dispersion (but averaged over the space).

3) Inclusion of inhomogeneous (evanescent) waves in lossless media that means solutions of the wave equations where both the mean wave vector $\boldsymbol{k}_{0}$ and, possibly, the mean frequency $\omega_{0}$ are complex quantities (is more a technical than a principal problem).

4) For homogeneous media with losses one cannot derive exact differential conservation theorems but one can derive (not in fully unique way) equations of the kind

$$
\nabla_{\lambda} T_{\kappa \lambda}(r)=f_{\kappa}(r)
$$

or in three-dimensional separation with $f_{\kappa}(r)=\left(f_{k}(\boldsymbol{r}, t), \frac{\mathrm{i}}{c} q(\boldsymbol{r}, t)\right)$

$$
\nabla_{l} T_{k l}(\boldsymbol{r}, t)+\frac{\partial}{\partial t} g_{k}(\boldsymbol{r}, t)=f_{k}(\boldsymbol{r}, t), \quad \nabla_{l} S_{l}(\boldsymbol{r}, t)+\frac{\partial}{\partial t} w(\boldsymbol{r}, t)=q(\boldsymbol{r}, t),
$$

where $\boldsymbol{f}(\boldsymbol{r}, t)$ can be interpreted as a force density and $q(\boldsymbol{r}, t)$ as a density of loss or gain of electromagnetic energy.

5) Derivation of a local conservation theorem for inhomogeneous media with $\hat{\varepsilon}_{i j} \equiv \hat{\varepsilon}_{i j}\left(\boldsymbol{r}, \boldsymbol{r}^{\prime}, t, t^{\prime}\right)$. In this case local conservation of energy and momentum is not possible but the action conservation is possible (adiabatic invariants).

6) Specialization of permittivity tensor, for example, to polaritons with $\varepsilon_{i j}(\boldsymbol{k}, \omega)=\frac{\omega^{2}-\omega_{l}^{2}}{\omega^{2}-\omega_{t}^{2}} \delta_{i j}$.

7) Taking into account higher-order derivatives of the slowly varying electric field amplitudes.

8) Quantum-mechanical generalization.

\section{Conclusion}

In present article, we developed a relativistically covariant approach to the local conservation theorems for homogeneous anisotropic media with dispersion of general permittivity tensor $\varepsilon_{i j}(\boldsymbol{k}, \omega)$ and to the calculation of the four-dimensional energy-momentum tensor ${ }^{14}$. The limiting case to plane monochromatic waves is discussed and the results, in particular, are demonstrated for the special case of cold plasma. In the usual approach for such problems, the starting point is a Lagrange function, but this approach is hardly applicable with inclusion of the dispersion. Our calculations are made in a coordinate-invariant operator approach

${ }^{14}$ I used my unpublished paper in German from about 1979 (see Remark) which I translated in preparation to a Conference in 2004 into English (see [34]) and to which I added since this time new material not fully included here and I am convinced that the content contains elements worth to be published also now. 
with an operator equation only for the electric field and therefore with results which are basically expressed by the electric field.

\section{Remark}

A shorter paper of this theme (in particular, without any statements to difficulties for General relativity theory due to asymmetry of the energy-momentum tensor and to application of a plasma) written in German with nearly all basic formulae as now was made in about 1979 but was rejected from Editor of Annalen der Physik in GDR Professor Gustav Richter with wrong arguments. His main wrong argument was that in my formulae for the limiting case to plane monochromatic waves stands the derivative of the permittivity with respect to the frequency that he declared as wrong "for physical reason". However, the correctness of these formulae was already known from cited monographs and papers, in particular, of Landau and Lifshits and of Agranovich and Ginzburg. When I wanted to explain G. Richter who was also Member of our Institute at that time (in age of a few years below 65) in personal talk why mentioned formulae are correct for beams in limiting case he became very angry. Similar things happened shortly before when I wanted to publish my paper about generalized boundary conditions which, finally, was published after intervention by a prominent physicist of GDR from Editorial Board of "Annalen" and recently I published a continuation of this topic. When I tried to send the mentioned paper about boundary conditions to a Western journal I never got an answer. One could not check whether or not it was really sent since mail to Western countries went before this in an open couvert to the Chief and through Security or was a response withheld. I found now the hand-written comments of G. Richter and will pose them into my Home-page.

\section{Acknowledgements}

Shortly, after my active time in the Institute at the end of 1999, already as pensioner I went to an Optics Conference in Szeged (Hungary) and gave a lecture about the "Energy-momentum tensor for... dispersive media...". The difficulty here was that in the Proceedings after the conference only 6 pages in a given small Latex-frame should be published with which I have had great difficulties but the 9 pages which I sent were accepted. Thank you very much M.G. Benedict from Szeged! A further forum in the new decennary I found also in the Group of Peřinová and Peřina from Olomouc where I could give a seminar lecture about mentioned topics. Thank you also very much!

\section{Conflicts of Interest}

The author declares no conflicts of interest regarding the publication of this paper.

\section{References}

[1] Einstein, A. (1913) Zeitschrift für Angewandte Mathematik und Physik, 62, 
225-261.

[2] Tamm, I.E., Smorodinsky, J.A. and Kuznetsov, B.G. (1965) Albert Einstein, Sobranyje nauchnykh truduv, I (Collection of Scientific Works, Part I). Nauka, Moskva.

[3] Landau, L.D. and Lifshitz, E.M. (1962) The Classical Theory of Fields (4th Revised Edition). Nauka, Moskva.

[4] Rashevski, P.K. (1964) Riemann Geometry and Tensor Analysis. 2nd Edition, Nauka, Moskva. (In Russian)

[5] Pauli, W. (1921) Relativitatstheorie. Enzyklopadie der mathematischen Wissenschaften, Band V, Heft IV, Art. 19, B.G. Teubner, Leipzig.

[6] Lorentz, H.A., Weyl, H. and Minkowski, H. (1922) Das Relativitatsprinzip, B.G. Teubner, Leipzig. https://doi.org/10.1007/978-3-663-16170-7

[7] Landau, L.D. and Lifshitz, E.M. (1982) Electrodynamics of Continuous Media. 2nd Edition, Nauka, Moskva.

[8] Agranovich, V.M. and Ginzburg, V.L. (1962) Nauk, 76-77, 643, 663. https://doi.org/10.3367/UFNr.0076.196204b.0643

[9] Agranovich, V.M. and Ginzburg, V.L. (1979) Spatial Dispersion in Crystal Optics and the Theory of Excitons. Second Edition, Nauka, Moskva.

[10] Ginzburg, V.L. (1981) Theoretical Physics and Astrophysics. Nauka, Moskva.

[11] Silin, V.P. and Rukhadze, A.A. (1961) Elektromagnitnye svoistva plasma i plasmapodobnykh sred (Electromagnetic Properties of Plasma and Plasma-Like Media). Gosatomisdat, Moskva.

[12] Tolman, R.Ch. (1969) Relativity Thermodynamics and Cosmology. Clarendon Press, Oxford.

[13] Sommerfeld, A. (1961) Elektrodynamik (3. Auflage). Akad. Verlagsgesellschaft Geest and Portig, Leipzig.

[14] von Laue, M. (1955) Die Relativitatstheorie, vol. 1, Die spezielle Relativitatstheorie. 6th Edition, Vieweg, Braunschweig.

[15] Fock, V. (1961) Theory of Space, Time and Gravitation. 2nd Edition, Fizmatgiz, Moskva.

[16] Skobeltsyn, D.V. (1973) Uspekhi Fizicheskikh Nauk, 110, 253-292. https://doi.org/10.3367/UFNr.0110.197306d.0253

[17] Ginsburg, V.L. and Ugarov, V.A. (1976) Uspekhi Fizicheskikh Nauk, 118, 175-188. https://doi.org/10.3367/UFNr.0118.197601e.0175

[18] Ugarov, V.A. (1977) Spetsialnaya teoriya otnositel'nosti (Special Relativity Theory). 2nd Edition, Nauka, Moskva.

[19] Tamm, I.Ye. (1976) Osnovy teorii elektrichestva (Foundations of the Theory of Elctricity). 9th Edition, Nauka, Moskva.

[20] Møller, C. (1972) The Theory of Relativity. Clarendon Press, Oxford.

[21] Jackson, J.D. (1962) Classical Electrodynamics. John Wiley, New York. https://doi.org/10.1063/1.3057859

[22] Felsen, L.B. and Marcuvitz, N. (1973) Radiation and Scattering of Waves. Prentice-Hall, Englewood Cleffs.

[23] Yariv, A. and Yeh, P. (1984) Optical Waves in Crystals. Wiley-Interscience, New York.

[24] Melrose, D.B. and McPhedran, R.C. (1991) Electromagnetic Processes in Dispersive Media. Cambridge University Press, Cambridge. 
https://doi.org/10.1017/CBO9780511600036

[25] Jimenez, J.L. and Campos, I. (1995) The Balance Equations of Energy and Momentum in Classical Electrodynamics. In: Barrett, T.W. and Grimes, D.M., Eds., Advanced Electromagnetism, World Scientific, Singapore, 464-495. https://doi.org/10.1142/9789812831323 0016

[26] Schwinger, J., DeRaad Jr., L.L., Milton, K.A. and Tsai, W.-Y. (1998) Classical Electrodynamics. Westview Press (Perseus Books Group, U.S.A.), Boulder.

[27] Fyodorov, F.I. (1958) Optika Anisotropnykh Sred, Optics of Anisotropic Media. Izdatelstvo Akademya nauk BSSR, Minsk. (In Russian)

[28] Fyodorov, F.I. (1976) Teorya girotropii (Theroy of gyrotropy). Nauka i tekhnika, Minsk.

[29] Fyodorov, F.I. (1979) Gruppa lorentsa (Lorentz Group). Nauka, Moskva.

[30] Wünsche, A. (1970) Annals of Physics (Leipzig), 25, 201-214. https://doi.org/10.1002/andp.19704800207

[31] Wünsche, A. (1978) Annals of Physics (Leipzig), 35, 303-320. https://doi.org/10.1002/andp.19784900407

[32] Leonhardt, U. (2000) Physical Review A, 62, Article ID: 012111. https://doi.org/10.1103/PhysRevA.62.012111

[33] Leonhardt, U. and Piwnicki, P. (2001) Journal of Modern Optics, 48, 977-988. https://doi.org/10.1080/09500340108230969

[34] Wünsche, A. (2005) Acta Physica Hungarica B, 23, 151-162. https://doi.org/10.1556/APH.23.2005.3-4.5

[35] Lorentz, H.A. (1904) van Wetenschappen te Amsterdam, 6, 809.

[36] Tyapkin, A.A. (1973) The Principle of Relativity (with a Preface of D.I. Blokhintsev). Atomisdat, Moscow. ${ }^{15}$

[37] Lorentz, H.A. (1904) Weiterbildung der Maxwellschen Theorie. Elektronentheorie. In: Enzyklopadie der mathematischen Wissenschaften, Bd. 2, Teubner, Leipzig, Art. $14,245$.

[38] Lorentz, H.A. (1915) Theory of Electrons. 2nd Edition, Teubner, Leipzig.

[39] de Groot, S.R. and Suttorp, L.G. (1972) Foundations of Electrodynamics. North Holland Publ. Comp., Amsterdam.

[40] Bloembergen, N. (1965) Nonlinear Optics. W.A. Benjamin, Inc., New York.

[41] Wünsche, A. (1971) Monatsberichte der Deutschen Akademie der Wissenschaften zu Berlin, 13, 479-492.

[42] Hebenstreit, H. and Suchy, K. (1979) Zeitschrift für Naturforschung A, 34, 11471157. https://doi.org/10.1515/zna-1979-1001

[43] Minkowski, H. (1909) Physikalische Zeitschrift, 10, 104-111.

[44] Lorentz, H.A., Einstein, A., Minkowski, H. and Weyl, H. (1952) The Principle of Relativity. Dover, Mineola.

[45] Landau, L.D. and Lifshitz, E.M. (1988) The Classical Theory of Fields. 7nd Edition,

\footnotetext{
${ }^{15}$ In comparison to [6], this is a collection of original articles only to Special relativity theory (SRT) and contains in addition articles by H. Poincaré, H.A. Lorentz, M. Planck and some others than in [6] which, partially, are more difficult to find in the original and in a second part it contains articles to the history of the principle of relativity. The Editor of this collection is controversially judged because he tries to lower the main merits of Einstein in the development of SRT and to suggest that other scientists (e.g., H. Poincaré) made this earlier as Einstein that, however, is not convincing (see also [48]).
} 
Nauka, Moskva.

[46] Szivessy, G. (1928) Kristalloptik. In: Geiger, H. and Scheel, K., Eds., Handbuch der Physik, Band XX, Springer, Berlin, 635-904.

https://doi.org/10.1007/978-3-642-90780-7 11

[47] Born, M. and Wolf, E. (1999) Principles of Optics. Seventh Edition, Cambridge University Press, Cambridge.

[48] Ginzburg, V.L. (1974) Kak i kto sozdal teoriyu otnocitel'nosti? (How and Who Created Relativity Theory?) In: Einshteynovski sbornik (Einsteinian Anthology), Nauka, Moscow, 351-384. 


\section{Appendix A}

\section{Derivations to relativistic-covariant treatment of macroscopic electrody- namics of anisotropic dispersive media}

Macroscopic electrodynamics is relativistically invariant only for the vacuum that means for permittivity tensor $\varepsilon_{i j}(\boldsymbol{k}, \omega)=\varepsilon(\omega) \delta_{i j}$ with $\varepsilon(\omega)=1$. However, for general $\varepsilon_{i j}(\boldsymbol{k}, \omega)$ it can be formulated in relativistic covariant form according to Minkowski (1908) [7] (Eqs. (76.9)-(76.11)) and many others. This form was less appropriate for our derivations of the energy-momentum tensor. Our derivation rests more on the invariance of $\boldsymbol{B}^{2}-\boldsymbol{E}^{2}$ after Fourier transformationation from space-time to wavevector-frequency representation, e.g., [3] [13] [15].

The starting point is Equation (5.4) for the electric field of plane monochromatic waves with the definition (5.5) of the wave-equation operator that means

$$
L_{i j}(\boldsymbol{k}, \omega) E_{j}(\boldsymbol{k}, \omega)=0, \quad L_{i j}(\boldsymbol{k}, \omega) \equiv \frac{c^{2}}{\omega^{2}}\left(k_{i} k_{j}-\boldsymbol{k}^{2} \delta_{i j}\right)+\varepsilon_{i j}(\boldsymbol{k}, \omega) .
$$

As discussed, it contains the full information of the macroscopic electromagnetic field together with linear constitutive equations for homogeneous anisotropic dispersive media expressed by the permittivity tensor $\varepsilon_{i j}(\boldsymbol{k}, \omega) \equiv \delta_{i j}+4 \pi \chi_{i j}(\boldsymbol{k}, \omega)$ with $\chi_{i j}(\boldsymbol{k}, \omega)$ the general susceptibility tensor.

We consider an arbitrary special Lorentz transformation $\Lambda$ which transforms a space-time vector $r=\left(\begin{array}{c}r \\ r_{4}=\mathrm{i} c t\end{array}\right)$ in inertial system ${ }^{16} \mathcal{I}$ into a new space-time vector $r^{\prime}=\left(\begin{array}{c}r^{\prime} \\ r_{4}^{\prime}=\mathrm{i} c t\end{array}\right)$ in inertial system $\mathcal{I}^{\prime}$ according to

$$
\begin{aligned}
& r_{\mu}^{\prime}=\Lambda_{\mu v} r_{v}, \quad r_{v}=\left(\Lambda^{-1}\right)_{v \mu} r_{\mu}^{\prime}=r_{\mu}^{\prime} \Lambda_{\mu v}, \\
& \nabla_{\mu}=\Lambda_{\mu \nu} \nabla_{v}^{\prime}, \quad \nabla_{v}^{\prime}=\left(\Lambda^{-1}\right)_{v \mu} \nabla_{\mu}=\nabla_{\mu} \Lambda_{\mu v}, \quad\left(\Lambda_{\mu \lambda} \Lambda_{v \lambda}=\delta_{\mu v}\right) .
\end{aligned}
$$

where $\Lambda_{\mu v}(\boldsymbol{V})$ with $\boldsymbol{V}$ as the relative velocity of $\mathcal{I}^{\prime}$ in $\mathcal{I}$ possesses the well-known form

$$
\Lambda_{\mu v}(\boldsymbol{V})=\left(\begin{array}{cc}
\delta_{m n}+(\gamma-1) \frac{V_{m} V_{n}}{\boldsymbol{V}^{2}} & i \gamma \frac{V_{m}}{c} \\
-i \gamma \frac{V_{n}}{c} & \gamma
\end{array}\right), \quad \gamma \equiv \frac{1}{\sqrt{1-\frac{\boldsymbol{V}^{2}}{c^{2}}}} \geq 1,
$$

with $\Lambda_{\mu \nu}^{-1}(\boldsymbol{V})=\Lambda_{\mu \nu}(-\boldsymbol{V})$. For the 4-wave vector $k \equiv\left(\boldsymbol{k}, \mathrm{i} \frac{\omega}{c}\right)$ the analogous transformation formula

$$
k_{\mu}^{\prime}=\Lambda_{\mu \nu} k_{v}, \quad k_{v}=\left(\Lambda^{-1}\right)_{v \mu} k_{\mu}^{\prime}=k_{\mu}^{\prime} \Lambda_{\mu v},
$$

holds. The antisymmetric electromagnetic field tensor $F_{\mu \nu}(r) \equiv F_{\mu \nu}=-F_{v \mu}$ in space-time representation and separated in three-dimensional form together with the transformation relations is ( $\varepsilon_{l m n}$ three-dimensional Levi-Civita pseudo-tensor)

${ }^{16}$ Einstein denotes "inertial systems" with letter $K$, likely, from the German "Koordinatensystem". 


$$
F_{\mu \nu}=\left(\begin{array}{cc}
\varepsilon_{m n l} B_{l} & -\mathrm{i} E_{m} \\
\mathrm{i} E_{n} & 0
\end{array}\right), \quad F_{\kappa \lambda}^{\prime}=\Lambda_{\kappa \mu} \Lambda_{\lambda \nu} F_{\mu \nu},
$$

and the basic equations of macroscopic electrodynamics in arbitrary inertial systems can be written ( $\varepsilon_{\kappa \lambda \mu \nu}$ four-dimensional Levi-Civita pseudo-tensor)

$$
\varepsilon_{\kappa \lambda \mu \nu} \nabla_{\lambda} F_{\mu \nu}=0, \quad \nabla_{v} F_{\mu v}=4 \pi j_{\mu},
$$

where $j_{\mu}=j_{\mu}(r)$ is the 4 -vector of current density in space-time representation. We now make the transition to the Fourier transforms of the field functions (definitions see (3.4)).

From the transformation formula for the 4-vector of current density $j(k)=(\boldsymbol{j}(\boldsymbol{k}, \omega)$, ic $\rho(\boldsymbol{k}, \omega))$ using the definition (2.3) which leads to $j(k)=(-\mathrm{i} \omega \boldsymbol{P}(\boldsymbol{k}, \omega), c \boldsymbol{k} \boldsymbol{P}(\boldsymbol{k}, \omega))$, we obtain the following transformation formula for the polarization $\left(P_{k}(k) \equiv P_{k}(\boldsymbol{k}, \omega), P_{i}^{\prime}\left(k^{\prime}\right) \equiv P_{i}^{\prime}\left(\boldsymbol{k}^{\prime}, \omega^{\prime}\right)\right)$

$$
P_{i}^{\prime}\left(k^{\prime}\right)=\frac{\omega}{\omega^{\prime}}\left(\Lambda_{i k}+\mathrm{i} \frac{c}{\omega} \Lambda_{i 4} k_{k}\right) P_{k}(k)=-\mathrm{i} \frac{c}{\omega^{\prime}} k_{\mu}^{\prime}\left(\Lambda_{i k} \Lambda_{\mu 4}-\Lambda_{i 4} \Lambda_{\mu k}\right) P_{k}\left(\Lambda^{-1} k^{\prime}\right) .
$$

After transformation of the known formula for the tensor of the electromagnetic field into a corresponding formula for the Fourier components and then after the elimination of the magnetic field by means of the first vectorial equation in (2.7) we arrive after some intermediate calculations to the following transformation formula for the electric field $\left(E_{l}(k) \equiv E_{l}(\boldsymbol{k}, \omega), E_{j}^{\prime}\left(k^{\prime}\right) \equiv E_{\boldsymbol{j}}^{\prime}\left(\boldsymbol{k}^{\prime}, \omega^{\prime}\right)\right)$

$$
E_{l}(k)=E_{l}\left(\Lambda^{-1} k^{\prime}\right)=-\mathrm{i} \frac{c}{\omega^{\prime}} k_{v}^{\prime}\left(\Lambda_{v 4} \Lambda_{j l}-\Lambda_{v l} \Lambda_{j 4}\right) E_{j}^{\prime}\left(k^{\prime}\right) .
$$

An analogous formula which connects alone the components of the magnetic field before and after the Lorentz transformation does not exist without using the permittivity tensor but one may calculate the magnetic field from the electric field in each system via the Fourier-transformed Maxwell equation $B_{j}(k)=\frac{c}{\omega} \varepsilon_{j k l} k_{k} E_{l}(k)$ and one can widely work with the electric field alone.

From the definition of the susceptibility tensor before and after the Lorentz transformation

$$
P_{k}(k)=\chi_{k l}(k) E_{l}(k), \quad P_{i}^{\prime}\left(k^{\prime}\right)=\chi_{i k}^{\prime}\left(k^{\prime}\right) E_{j}^{\prime}\left(k^{\prime}\right),
$$

using (A.7) and (A.8) we obtain the following transformation formula

$$
\chi_{i j}^{\prime}\left(k^{\prime}\right)=-\frac{c^{2}}{\omega^{\prime 2}} k_{\mu}^{\prime} k_{v}^{\prime}\left(\Lambda_{\mu 4} \Lambda_{i k}-\Lambda_{\mu k} \Lambda_{i 4}\right)\left(\Lambda_{v 4} \Lambda_{j l}-\Lambda_{v l} \Lambda_{j 4}\right) \chi_{k l}\left(\Lambda^{-1} k^{\prime}\right) .
$$

Using the definition of the permittivity tensor before and after the Lorentz transformation, we obtain from (A.10) the corresponding transformation formula for the permittivity tensor

$$
\varepsilon_{k l}(k) \equiv \delta_{k l}+4 \pi \chi_{k l}(k), \quad \varepsilon_{i j}^{\prime}\left(k^{\prime}\right) \equiv \delta_{i j}+4 \pi \chi_{i j}^{\prime}\left(k^{\prime}\right) .
$$

Thus in the inertial system $\mathcal{I}^{\prime}$ the medium appears as a homogeneous anisotropic and dispersive one with the permittivity tensor $\varepsilon_{i j}^{\prime}\left(k^{\prime}\right) \equiv \varepsilon_{i j}^{\prime}\left(\boldsymbol{k}^{\prime}, \omega^{\prime}\right)$.

For a three-dimensional orthogonal transformation 


$$
\Lambda_{\mu v}=\left(\begin{array}{cc}
\Lambda_{m n} & \Lambda_{m 4} \\
\Lambda_{4 n} & \Lambda_{44}
\end{array}\right)=\left(\begin{array}{cc}
R_{m n} & 0 \\
0 & 1
\end{array}\right), \quad\left(R_{m l} R_{n l}=\delta_{m n}\right),
$$

we find the usual transformation formula of a second-rank tensor function

$$
\chi_{i j}^{\prime}\left(\boldsymbol{k}^{\prime}, \omega^{\prime}\right)=R_{i k} R_{j l} \chi_{k l}\left(\mathrm{R}^{-1} \boldsymbol{k}^{\prime}, \omega^{\prime}\right),
$$

where $\mathrm{R}=\left(\mathrm{R}^{\mathrm{T}}\right)^{-1}$ denotes the three-dimensional rotation operator.

The transformation of space-time vectors after separation of a part parallel and perpendicular of $\boldsymbol{r}$ to the velocity $\boldsymbol{V}$ can be written

$$
\boldsymbol{r}^{\prime}=\boldsymbol{r}+V\left((\gamma-1) \frac{\boldsymbol{V} \boldsymbol{r}}{\boldsymbol{V}^{2}}-\gamma t\right), \quad t^{\prime}=\gamma\left(t-\frac{\boldsymbol{V} \boldsymbol{r}}{c^{2}}\right),
$$

and, correspondingly, of wave vector and frequency according to (see (A.4) together with (A.3))

$$
\boldsymbol{k}^{\prime}=\boldsymbol{k}+\left((\gamma-1) \frac{\boldsymbol{k} \boldsymbol{V}}{\boldsymbol{V}^{2}}-\gamma \frac{\omega}{c^{2}}\right) \boldsymbol{V}, \quad \omega^{\prime}=\gamma(\omega-\boldsymbol{k} \boldsymbol{V}),
$$

with relativistic invariants

$$
\boldsymbol{r}^{\prime 2}-c^{2} t^{\prime 2}=\boldsymbol{r}^{2}-c^{2} t^{2}, \quad \boldsymbol{k}^{\prime 2}-\frac{\omega^{\prime 2}}{c^{2}}=\boldsymbol{k}^{2}-\frac{\omega^{2}}{c^{2}}, \quad \boldsymbol{k}^{\prime} \boldsymbol{r}^{\prime}-\omega^{\prime} t^{\prime}=\boldsymbol{k} \boldsymbol{r}-\omega t .
$$

Furthermore from (A.15) follows, in particular

$$
\left[\boldsymbol{k}^{\prime}, \boldsymbol{V}\right]=[\boldsymbol{k}, \boldsymbol{V}], \quad \boldsymbol{k}^{\prime} \boldsymbol{V}=\gamma\left(\boldsymbol{k} \boldsymbol{V}-\omega \frac{\boldsymbol{V}^{2}}{c^{2}}\right)=\gamma(\boldsymbol{k} \boldsymbol{V}-\omega)+\frac{\omega}{\gamma} .
$$

As expected this shows that the components of the wave vector $\boldsymbol{k}$ perpendicular to the velocity $\boldsymbol{V}$ of the inertial system $\mathcal{I}^{\prime}$ in $\mathcal{I}$ is not influenced by the transformation that, clearly, is known. The transformation formulae (A.7) and (A.8) in three-dimensional separation take on the form

$$
P_{i}^{\prime}\left(\boldsymbol{k}^{\prime}, \omega^{\prime}\right)=\left\{\frac{V_{i} V_{k}}{\boldsymbol{V}^{2}}+\gamma\left(\delta_{i k}-\frac{V_{i} V_{k}}{\boldsymbol{V}^{2}}+\frac{\boldsymbol{k}^{\prime} \boldsymbol{V} \delta_{i k}-V_{i} k_{k}^{\prime}}{\omega^{\prime}}\right)\right\} P_{k}(\boldsymbol{k}, \omega),
$$

and using $\boldsymbol{B}^{\prime}\left(\boldsymbol{k}^{\prime}, \omega^{\prime}\right)=\frac{c}{\omega^{\prime}}\left[\boldsymbol{k}^{\prime}, \boldsymbol{E}^{\prime}\left(\boldsymbol{k}^{\prime}, \omega^{\prime}\right)\right]$ (cf. also with formulae (8.2) in [42])

$$
E_{l}(\boldsymbol{k}, \omega)=\left\{\frac{V_{l} V_{j}}{\boldsymbol{V}^{2}}+\gamma\left(\delta_{l j}-\frac{V_{l} V_{j}}{\boldsymbol{V}^{2}}+\frac{\boldsymbol{k}^{\prime} \boldsymbol{V} \delta_{l j}-k_{l}^{\prime} V_{j}}{\omega^{\prime}}\right)\right\} E_{j}^{\prime}\left(\boldsymbol{k}^{\prime}, \omega^{\prime}\right),
$$

where, in addition, the transformations (A.15) for the arguments have to be used. For the general susceptibilities defined by (A.9) using (A.18) and A.19) this leads finally to the transformation formula to the moving medium

$$
\begin{aligned}
\chi_{i j}^{\prime}\left(\boldsymbol{k}^{\prime}, \omega^{\prime}\right)= & \left\{\frac{V_{i} V_{k}}{\boldsymbol{V}^{2}}+\gamma\left(\delta_{i k}-\frac{V_{i} V_{k}}{\boldsymbol{V}^{2}}+\frac{\boldsymbol{k}^{\prime} \boldsymbol{V} \delta_{i k}-V_{i} k_{k}^{\prime}}{\omega^{\prime}}\right)\right\} \\
& \cdot\left\{\frac{V_{j} V_{l}}{\boldsymbol{V}^{2}}+\gamma\left(\delta_{j l}-\frac{V_{j} V_{l}}{\boldsymbol{V}^{2}}+\frac{\boldsymbol{k}^{\prime} \boldsymbol{V} \delta_{j l}-V_{j} k_{l}^{\prime}}{\omega^{\prime}}\right)\right\} \\
& \cdot \chi_{k l}(\underbrace{\boldsymbol{k}^{\prime}+(\gamma-1) \frac{\boldsymbol{k}^{\prime} \boldsymbol{V}}{\boldsymbol{V}^{2}} \boldsymbol{V}+\gamma \frac{\omega^{\prime}}{c^{2}} \boldsymbol{V}}_{=\boldsymbol{k}}, \underbrace{\gamma\left(\omega^{\prime}+\boldsymbol{k}^{\prime} \boldsymbol{V}\right)}_{=\omega}) .
\end{aligned}
$$


The transformation of the corresponding permittivity tensors follow from

$$
\varepsilon_{i j}^{\prime}\left(\boldsymbol{k}^{\prime}, \omega^{\prime}\right) \equiv \delta_{i j}+4 \pi \chi_{i j}^{\prime}\left(\boldsymbol{k}^{\prime}, \omega^{\prime}\right), \quad \varepsilon_{k l}(\boldsymbol{k}, \omega) \equiv \delta_{k l}+4 \pi \chi_{k l}(\boldsymbol{k}, \omega) .
$$

If $\chi_{i j}(\boldsymbol{k}, \omega)$ is a symmetric tensor then $\chi_{i j}^{\prime}\left(\boldsymbol{k}^{\prime}, \omega^{\prime}\right)$ is also a symmetric tensor. Furthermore, we see that both indices of these tensors are independently transformed by exactly the same tensorial factors in (A.20) written on first and second lines.

From the transformation formulae we find then the formal Lorentz covariance of the expression

$I_{1} \equiv E_{i}( \pm k) E_{i}(k)-B_{i}( \pm k) B_{i}(k)=E_{i}( \pm k) \frac{c^{2}}{\omega^{2}} k_{\mu} k_{v}\left(\delta_{\mu i} \delta_{v j}-\delta_{\mu v} \delta_{i j}\right) E_{j}(k),(A$

and the formal covariance of

$$
I_{2} \equiv 4 \pi E_{i}( \pm k) \chi_{i j}(k) E_{j}(k) .
$$

The sum of both these covariant expressions can be written by means of the operator $L_{i j}(k)$ introduced in (2.7) as follows

$$
I \equiv I_{1}+I_{2}=E_{i}( \pm k) L_{i j}(k) E_{j}(k) .
$$

Thus the basis of the presented concept of relativistic-covariant treatment of electrodynamics and optics of homogeneous dispersive media is formed by the vectorial wave equation for the electric field (5.4) in the form $L_{i j}(k) E_{j}(k)=0$ together with one of the two equivalent representations

$$
L_{i j}(k) \equiv \frac{c^{2}}{\omega^{2}} k_{\mu} k_{v}\left(\delta_{\mu i} \delta_{v j}-\delta_{\mu v} \delta_{i j}\right)+4 \pi \chi_{i j}(k)=\frac{c^{2}}{\omega^{2}}\left(k_{i} k_{j}-\boldsymbol{k}^{2} \delta_{i j}\right)+\varepsilon_{i j}(\boldsymbol{k}, \omega),
$$

in connection with the transformation formulae (A.8) for the electric field and for the tensor of susceptibility (A.10) as well as the use of the formal Lorentz invariance of the quantities considered in (A.22) and (A.24). The material properties are described by only one susceptibility tensor which depends on wave vector $\boldsymbol{k}$ and frequency $\omega$ before and after transformation from systems $\mathcal{I}$ to $\mathcal{I}^{\prime}$.

One has to pay attention that for reason of consideration of the susceptibility tensor the transformation (A.18) is given in direction $\mathcal{I} \rightarrow \mathcal{I}^{\prime}$ and the transformation (A.19) in inverse direction $\mathcal{I}^{\prime} \rightarrow \mathcal{I}$ and that the transformation operators are almost but not fully equal where both are represented using $\boldsymbol{k}^{\prime}$ and $\omega^{\prime}$ in system $\mathcal{I}^{\prime}$. These formulae describe the relativistic Doppler effect. The inverse formulae to (A.18), (A.19) and (A.15) and of transformation formulae in further text are obtained by substituting $\boldsymbol{V} \rightarrow-\boldsymbol{V}$ and by interchanging all quantities with and without primes. In contrast to (A.19) and (A.18) the well-known transformation formulae of Minkowski (e.g., [7], $₫ 76$ or, e.g., [5] [18]) are mixed transformations between electric and magnetic field which, furthermore, neglect the dispersion and are not made for the Fourier transforms of the fields. 


\section{Appendix B}

\section{Special Lorentz transformation of the energy-momentum tensor}

In the transition from inertial system $\mathcal{I}$ to inertial system $\mathcal{I}^{\prime}$ moving with velocity $\boldsymbol{V}$ in $\mathcal{I}$ the energy-momentum tensor transforms written in tensorial and in matrix form and as similarity transformation (upper index $\mathrm{T}$ means transposed)

$$
T_{\kappa \lambda}^{\prime}=\Lambda_{\kappa \mu} \Lambda_{\lambda \nu} T_{\mu \nu}=\Lambda_{\kappa \mu} T_{\mu \nu} \Lambda_{\nu \lambda}^{\mathrm{T}}=\Lambda_{\kappa \mu} T_{\mu \nu} \Lambda_{\nu \lambda}^{-1}, \quad\|\Lambda\|=1,
$$

that for the special Lorentz transformation (A.3) in space-time separation according to $(8.1)$ is

$$
\begin{aligned}
T_{k l}^{\prime}= & \left.\delta_{k m}+(\gamma-1) \frac{V_{k} V_{m}}{\boldsymbol{V}^{2}}\right)\left(\left(\delta_{l n}+(\gamma-1) \frac{V_{l} V_{n}}{\boldsymbol{V}^{2}}\right) T_{m n}-\gamma V_{l} g_{m}\right) \\
& -\gamma \frac{V_{k}}{c^{2}}\left(\left(\delta_{l n}+(\gamma-1) \frac{V_{l} V_{n}}{\boldsymbol{V}^{2}}\right) S_{n}-\gamma V_{l} w\right), \\
g_{k}^{\prime}= & \gamma\left\{-\left(\delta_{k m}+(\gamma-1) \frac{V_{k} V_{m}}{\boldsymbol{V}^{2}}\right)\left(\frac{V_{n}}{c^{2}} T_{m n}-g_{m}\right)+\gamma \frac{V_{k}}{c^{2}}\left(\frac{V_{n}}{c^{2}} S_{n}-w\right)\right\}, \\
S_{l}^{\prime}= & \gamma\left\{-\left(\delta_{l n}+(\gamma-1) \frac{V_{l} V_{n}}{\boldsymbol{V}^{2}}\right)\left(V_{m} T_{m n}-S_{n}\right)+\gamma V_{l}\left(V_{m} g_{m}-w\right)\right\}, \\
w^{\prime}= & \gamma^{2}\left\{\frac{V_{n}}{c^{2}}\left(V_{m} T_{m n}-S_{n}\right)-\left(V_{m} g_{m}-w\right)\right\} .
\end{aligned}
$$

From this follows

$$
\begin{aligned}
& T_{k l}^{\prime}-T_{l k}^{\prime}=\left(\delta_{k m}+(\gamma-1) \frac{V_{k} V_{m}}{\boldsymbol{V}^{2}}\right)\left(\delta_{l n}+(\gamma-1) \frac{V_{l} V_{n}}{\boldsymbol{V}^{2}}\right)\left(T_{m n}-T_{n m}\right) \\
&+\gamma\left\{V_{k}\left(g_{l}-\frac{1}{c^{2}} S_{l}\right)-V_{l}\left(g_{k}-\frac{1}{c^{2}} S_{k}\right)\right\}, \\
& g_{k}^{\prime}-\frac{1}{c^{2}} S_{k}^{\prime}=\gamma\left\{\frac{V_{m}}{c^{2}}\left(T_{m k}-T_{k m}\right)+\left(\delta_{k m}-\frac{V_{k} V_{m}}{V^{2}}\right)\left(g_{m}-\frac{1}{c^{2}} S_{m}\right)\right\},
\end{aligned}
$$

showing that symmetric and anti-symmetric parts of the energy-momentum tensor transforms independently on each other.

If we denote the energy-momentum tensor by a four-dimensional matrix $T$ and the Lorentz transformation by a matrix $\Lambda$ then the transformation (B.1) with $\Lambda^{\mathrm{T}}=\Lambda^{-1}$ (Minkowski metrics $g_{\mu v}=\delta_{\mu v}$ ) can be written as a similarity transformation as follows

$$
T^{\prime}=\Lambda T \Lambda^{-1} .
$$

This shows that the real-valued independent invariants of the energy-momentum tensor with respect to Lorentz transformations are the invariants of the similarity transformations (B.4) which can be chosen as the coefficients in the fourdimensional Hamilton-Cayley identity for $T$

$$
T^{4}-\langle T\rangle T^{3}+[T] T^{2}-|T| T+\|T\| I=0,
$$

where the first three invariants with respect to similarity transformations $\langle T\rangle,[T],|T|$ are formally given by the same formulae as for three-dimensional 
operators but, e.g., with $\langle T\rangle$ the four-dimensional trace of $T$ and, in addition, we have to consider the four-dimensional determinant $\|T\|$ that means

$$
\begin{aligned}
& \langle T\rangle \equiv T_{\mu}^{\mu}, \quad[T]=\frac{1}{2}\left(\langle T\rangle^{2}-\left\langle T^{2}\right\rangle\right), \quad|T|=\frac{1}{6}\left(\langle T\rangle^{3}-3\langle T\rangle\left\langle T^{2}\right\rangle+2\left\langle T^{3}\right\rangle\right) \\
& \|T\|=\frac{1}{24}\left(\langle T\rangle^{4}-6\langle T\rangle^{2}\left\langle T^{2}\right\rangle+3\left\langle T^{2}\right\rangle^{2}+8\langle T\rangle\left\langle T^{3}\right\rangle-6\left\langle T^{4}\right\rangle\right) .
\end{aligned}
$$

We now use an index-less representation of the energy-momentum tensor $T$ of the form ${ }^{17}$

$$
T \equiv\left(\begin{array}{cc}
\mathrm{T} & \mathrm{i} c \boldsymbol{g} \\
\frac{\mathrm{i}}{c} & -w
\end{array}\right),
$$

with $\mathrm{T}$ the stress tensor $\mathrm{T}$ (or its matrix) as a three-dimensional operator and find for the four-dimensional invariants of $T$ expressed by the three-dimensional invariants formed from the three-dimensional operator T, from the vectors $g$ and $\boldsymbol{S}$ and from the scalar $w$ in (B.7)

$$
\begin{aligned}
& \langle T\rangle=\langle\mathrm{T}\rangle-w, \\
& {[T]=[\mathrm{T}]-\langle\mathrm{T}\rangle w+\boldsymbol{S g},} \\
& |T|=|\mathrm{T}|-[\mathrm{T}] w+\langle\mathrm{T}\rangle \boldsymbol{S g}-\boldsymbol{S T} \boldsymbol{g}, \\
& \|T\|=-|\mathrm{T}| w+[\mathrm{T}] \boldsymbol{S g}-\langle\mathrm{T}\rangle \boldsymbol{S T} \boldsymbol{g}+\boldsymbol{S} \mathrm{T}^{2} \boldsymbol{g},
\end{aligned}
$$

where $\langle\mathrm{A}\rangle$ denotes the trace of an arbitrary three-dimensional operator $A$, [A] its second invariant and $|\mathrm{A}|$ its determinant according to

$$
\begin{aligned}
& \langle\mathrm{A}\rangle \equiv \frac{1}{2} \varepsilon_{i j k} \varepsilon_{l j k} A_{i l}=A_{k k}, \quad[\mathrm{~A}] \equiv \frac{1}{2} \varepsilon_{i j k} \varepsilon_{l m k} A_{i l} A_{j m}=\frac{1}{2}\left(\langle\mathrm{~A}\rangle^{2}-\left\langle\mathrm{A}^{2}\right\rangle\right), \\
& |\mathrm{A}| \equiv \frac{1}{6} \varepsilon_{i j k} \varepsilon_{l m n} A_{i l} A_{j m} A_{k n}=\frac{1}{6}\left(\langle\mathrm{~A}\rangle^{3}-3\langle\mathrm{~A}\rangle\left\langle\mathrm{A}^{2}\right\rangle+2\left\langle\mathrm{~A}^{3}\right\rangle\right),
\end{aligned}
$$

and where $\boldsymbol{x} \boldsymbol{y} \equiv x_{n} y_{n}$ is the scalar product of three-dimensional vectors $\boldsymbol{x}$ with $\boldsymbol{y}$ and $\boldsymbol{x} \mathrm{A} \boldsymbol{y} \equiv x_{m} A_{m n} y_{n}$ a three-dimensional bilinear form. The first invariant $\langle T\rangle$ is the already mentioned trace of $T$ and the invariant $\|T\|$ the determinant of the 4-dimensional energy-momentum tensor. All are also relativistic invariants due to (B.4). Among the invariants (B.8) one has only one invariant which is linear in the components of the energy-momentum tensor $T$, namely, the trace $\langle T\rangle=\langle\mathrm{T}\rangle-w$.

According to (9.9) the trace $\langle T\rangle$ does not vanish in general but it vanishes in case of neglected dispersion. The second invariant $[T]$ and the higher invariants vanish for energy-momentum tensors which factorize in the form of dyadic products of two vectors. This means that one has only one non-vanishing eigenvalue of this tensor also if one takes into account the dispersion as it is shown for the second invariant in (12.15). One has to expect that the second and the higher invariants of the energy-momentum tensor do not vanish in higher approximations of the slowly varying amplitudes as it is suggested by considerations in Section 9.

${ }^{17}$ We remind that the spatial part $\mathrm{T}$ and $T$ itself are not uniquely defined in literature with respect to sign and also to their notation. 